\title{
Checklist of the Crustacea from the state of Santa Catarina, Brazil
}

\author{
Harry Boos ${ }^{1 *}$, Georgina Bond Buckup ${ }^{2}$, Ludwig Buckup ${ }^{2}$, Paula B. Araujo ${ }^{2}$, Célio Magalhães ${ }^{3}$, \\ Mauricio P. Almerão ${ }^{2}$, Roberta A. dos Santos ${ }^{1}$ and Fernando L. Mantelatto ${ }^{4}$ \\ 1 Instituto Chico Mendes de Conservação da Biodiversidade, Centro de Pesquisa e Gestão de Recursos Pesqueiros do Litoral Sudeste e Sul. Av \\ Ministro Victor Konder, $n^{\circ}$ 374. CEP 88301-700. Itajaí, SC, Brazil. \\ 2 Universidade Federal do Rio Grande do Sul, Instituto de Biociências, Departamento de Zoologia, Programa de Pós-Graduação em Biologia Animal. \\ Av. Bento Gonçalves, 9500. CEP 43433 91501-970. Porto Alegre, RS, Brazil. \\ 3 Instituto Nacional de Pesquisas da Amazônia. Av. André Araújo, 2936. CEP 69060-001. Manaus, AM, Brazil. \\ 4 Universidade de São Paulo, Faculdade de Filosofia, Ciências e Letras de Ribeirão Preto, Departamento de Biologia, Laboratório de Bioecologia \\ e Sistemática de Crustáceos, Programa de Pós-Graduação em Ciências: Biologia Comparada, Av. Bandeirantes, 3.900. CEP 14040-900. Ribeirão \\ Preto, SP, Brazil. \\ * Corresponding author. E-mail: harry.boos-junior@icmbio.gov.br
}

\begin{abstract}
The list of Crustacean species from the state of Santa Catarina, southern Brazil, comprises 518 valid species, distributed in 17 orders and 152 families. This amount corresponds to $0.75 \%$ of the Crustacea worldwide and about $20 \%$ of Brazilian species. The order Decapoda is the most represented, with 280 species. The families of Decapoda with the greatest number of species recorded were: Aeglidae (19), Palaemonidae (13) and Portunidae (13). In addition, the occurrence of 19 species distributed in the orders Cyclopoida (1), Podocopida (2) and Decapoda (16) is reported for the first time. Exotic species (9) are distributed in four different orders - Isopoda (6), Calanoida (1), Thoracica (1) and Decapoda (1).
\end{abstract}

\section{INTRODUCTION}

The first studies on the crustacean fauna (carcinofauna) from the state of Santa Catarina were performed by Johann Friedrich Theodor Müller (1822-1897), better known as Fritz Müller (e.g. Müller 1862; 1876; 1880a; 1880b; 1881; 1892). Afterwards several researchers have been studying the carcinofauna in this region. According to the recent publication "Diagnóstico da Zoologia no Brasil", organized by the Brazilian Zoology Society - SBZ (Rocha and Boeger 2009), researchers of three scientific institutions based in Santa Catarina state have been working with carcinology: Universidade do Vale do Itajaí (UNIVALI), Universidade Federal de Santa Catarina (UFSC) and Centro de Pesquisa e Gestão de Recursos Pesqueiros do Litoral Sudeste e Sul (CEPSUL / ICMBio) (Bond-Buckup et al. 2009). However other research groups have considerably contributed to the knowledge of the crustacean diversity in this region.

The information on the occurrence of the crustaceans in the state of Santa Catarina is dispersed and available in books, articles, catalogues, theses, foundation reports and collections, and many of them have not been published or remain as gray literature. Given this lack of a comprehensive data set about this state's carcinofauna, data were compiled from publications and collections in order to elaborate the present list of its crustaceans.

\section{MATERIALS AND MethodS}

In general, the crustacean species' classification was made according to Martin and Davis (2001), Appeltans et al. (2011) and the Integrated Taxonomic Information System (2010) (http://www.itis.gov). For some groups, the following specific classifications were used: for general Decapoda (De Grave et al. 2009) and specific groups as for Galatheidae (Baba et al. 2008), for Hippoidea (Boyko and McLaughlin 2010), for Paguroidea, Hippoidea and Porcellanidae (McLaughlin et al. 2010a), for Aeglidae (Bond-Buckup 2003; McLaughlin et al. 2010b), for Astacidea, Achelata and Polychelida (Chan 2010), for Brachyura (Ng et al. 2008), for Trichopeltariidae (Tavares and Cleva 2010), for Dendrobranchiate and Caridean Shrimps (De Grave and Fransen 2011; Anker 2012).

The occurrence of a species in the state of Santa Catarina is based either in some of records mentioned previously in publications or in specimens deposited in institutional collections which were accessed by the authors (in such case, the locality data, and the respective collection acronym and catalogue number are informed). Some records based only in compilation papers (catalogue, manuals of identification, academic papers) that have not informed specimens or collection data should be taken with caution, as they were not checked by the authors.

The location of the terrestrial species from the infraorder Isopoda was defined by the city where the sampling occurred. In freshwater species, the watercourse was indicated and, in some cases, both the watershed and the geographical coordinates were mentioned. The information on depth (when available) was also indicated for the marine species. This heterogeneity of information was due to the source's data available. In the present checklist, the following institutions with respective abbreviations were cited:

CEPSUL: Coleção do Centro de Pesquisa e Gestão de Recursos Pesqueiros do Litoral Sudeste e Sul, Itajaí.

CCDB: Coleção de Crustáceos do Departamento de Biologia da FFCLRP, Universidade de São Paulo, Ribeirão Preto.

DZ-UFPR: Coleção do Departamento de Zoologia da Universidade Federal do Paraná, Curitiba. 
DOUFPe: Coleção de Crustáceos do Departamento de Oceanografia da Universidade Federal de Pernambuco, Recife.

FURG: Universidade Federal de Rio Grande, Rio Grande. INPA: Instituto Nacional de Pesquisas da Amazônia, Manaus.

MCSN: Museo Civico di Storia Naturale, Milano.

MCT-PUCRS: Museu de Ciências e Tecnologia, Ponticífia Universidade Católica do Rio Grande do Sul, Porto Alegre.

MHNCI: Museu de História Natural Capão da Imbuia, Curitiba.

MHNG: Muséum d'Histoire Naturelle, Genève.

MNRJ: Museu Nacional, Universidade Federal do Rio de Janeiro, Rio de Janeiro.

MZUSP: Museu de Zoologia, Universidade de São Paulo, São Paulo.

NHM: The Natural History Museum, London

NHMW: Naturhistorisches Museum, Vienna.

SMF: Senckenberg Museum, Frankfurt.

SMNH: Swedish Museum of Natural History, Stockholm. UFRGS: Coleção do Departamento de Zoologia,

Universidade Federal do Rio Grande do Sul, Porto Alegre.

USNM: National Museum of Natural History, Smithsonian Institution, Washington, D.C.

ZMB: Zoologisches Museum der Humboldt Universität, Berlin.

ZMH: Zoologisches Museum, Universität Hamburg, Hamburg.

\section{RESULTS AND DISCUSSION}

The crustacean species' list from the state of Santa Catarina comprises 518 valid species, distributed in 17 orders (Table 1) and 152 families. This amount corresponds to $0.75 \%$ of the Crustacea worldwide valid species and about 20\% of Brazilian species (Martin and Davis 2001; Amaral and Jablonski 2005).

The checklist of crustacean species from Santa Catarina state is shown below divided into classes, orders and families, following alphabetical order.

FIGURE 1. Crustaceans orders and number of species from Santa Catarina, Brazil.

\begin{tabular}{lc}
\hline ORDER & NUMBER OF SPECIES \\
\hline Amphipoda & 12 \\
Arguloidea & 1 \\
Calanoida & 40 \\
Cyclopoida & 25 \\
Decapoda & 280 \\
Diplostraca & 34 \\
Harpacticoida & 28 \\
Isopoda & 44 \\
Kentrogonida & 3 \\
Lepadiformes & 4 \\
Mysida & 1 \\
Podocopida & 7 \\
Poecilostomatoida & 8 \\
Scalpelliformes & 4 \\
Sessilia & 15 \\
Siphonostomatoida & 4 \\
Stomatopoda & 8 \\
\hline TOTAL & $\mathbf{5 1 8}$ \\
\hline
\end{tabular}

Crustacea Brünnich, 1772

Branchiopoda Latreille, 1817

Diplostraca Gerstaecker, 1866

Bosminidae Baird, 1845

Bosmina hagmanni Stingelin, 1904

Previous record: Serafim-Júnior et al. 2006.

Bosmina longirostris (0. F. Müller, 1785)

Previous record: Serafim-Júnior et al. 2006; Resgalla Jr. et al. 2008; Veado et al. 2010.

Bosmina tubicen Brehm, 1953

Previous record: Serafim-Júnior et al. 2006.

Bosminopsis deitersi Richard, 1895

Previous record: Serafim-Júnior et al. 2006; Veado et al. 2010.

Eubosmina huaronensis (Delachaux, 1918)

Previous record: Serafim-Júnior et al. 2006 (cited as Bosmina huaronensis).

\section{Chydoridae Stebbing, 1902}

Alona guttata G. 0. Sars, 1862

Previous record: Serafim-Júnior et al. 2006.

Alona intermedia G. 0. Sars, 1862

Previous record: Serafim-Júnior et al. 2006.

Alona monacantha Sars, 1901

Previous record: Serafim-Júnior et al. 2006.

Camptocercus australis Sars, 1896

Previous record: Veado et al. 2010 (cited as Camptocercus dadayi Stingelin, 1913).

Chydorus eurynotus Sars, 1901

Previous record: Serafim-Júnior et al. 2006; Veado et al. 2010.

Chydorus nitidulus Sars, 1901

Previous record: Serafim-Júnior et al. 2006.

Chydorus sphaericus (O.F. Müller, 1776)

Previous record: Elmoor-Loureiro 2000.

Disparalona dadayi (Birge, 1910)

Previous record: Serafim-Júnior et al. 2006.

Euryalona orientalis (Daday, 1898)

Previous record: Veado et al. 2010.

Kurzia latissima (Kurz, 1874)

Previous record: Veado et al. 2010.

Leydigiopsis brevirostris Brehm, 1938

Previous record: Serafim-Júnior et al. 2006.

Oxyurella longicaudis (Birge, 1910)

Previous record: Veado et al. 2010.

Daphniidae Straus, 1820

Ceriodaphnia pulchella Sars, 1862

Previous record: Elmoor-Loureiro 2000.

Ceriodaphnia quadrangula (O.F. Müller, 1785)

Previous record: Veado et al. 2010.

Ceriodaphnia reticulata (Jurine, 1820)

Previous record: Serafim-Júnior et al. 2006.

Ceriodaphnia silvestrii Daday,1902

Previous record: Serafim-Júnior et al. 2006; Resgalla Jr. et al. 2008. 
Daphnia gessneri Herbst, 1967

Previous record: Serafim-Júnior et al. 2006.

Daphnia laevis Birge, 1878

Previous record: Veado et al. 2010.

Simocephalus vetulus (0. F. Muller.1776)

Previous record: Veado et al. 2010.

Ilyocryptidae Smirnov,1992

Ilyocryptus spinifer Herrick, 1882

Previous record: Serafim-Júnior et al. 2006; Veado et al. 2010.

Macrothricidae Norman and Brady, 1867

Macrothrix mira (Smirnov, 1982)

Previous record: Serafim-Júnior et al. 2006.

Macrothrix triserialis (Brady, 1886)

Previous record: Resgalla Jr. et al. 2008; Veado et al. 2010.

Moinidae Goulden, 1968

\section{Moina micrura Kurz, 1874}

Previous record: Serafim-Júnior et al. 2006.

Moina minuta Hansen, 1899

Previous record: Resgalla Jr. et al. 2008; Veado et al. 2010.

Podonidae Mordukhai-Boltovskoi, 1968

Evadne spinifera P. E. Müller, 1867

Previous record: Montú and Gloeden 1998; Resgalla Jr. et al. 2008.

Pleopis polyphaemoides (Leuckart,1859)

Previous record: Resgalla Jr. et al. 2008.

Podon intermedius Lilljeborg, 1853

Previous record: Montú and Gloeden 1998.

Pseudevadne tergestina (Claus, 1877)

Previous record: Montú and Gloeden 1998; Resgalla Jr. et al. 2008; Veado et al. 2010.

Sididae Baird, 1850

Penilia avirostris Dana, 1849

Previous record: Montú and Gloeden 1998; Resgalla Jr. et al. 2008; Veado et al. 2010.

Malacostraca Latreille, 1802

Amphipoda Latreille, 1816

Bateidae Stebbing, 1906

Batea catharinensis Müller, 1865

Previous record: Wakabara and Serejo 1998.

Caprellidae Leach, 1814

Caprella penantis Leach, 1814

Previous record: Müller and Mittmann 1998.

Caprella scaura Templeton, 1836

Previous record: Wakabara and Serejo 1998.

Paracaprella pusilla Mayer, 1890
Previous record: Wakabara and Serejo 1998.

Corophiidae Dana, 1849

Audulla chelifera Chevreux, 1901

Previous record: Wakabara and Serejo 1998; ValérioBerardo and Miyagi 2000.

Monocorophium acherusicum (Costa, 1857)

Previous record: Wakabara and Serejo 1998 [cited as

Corophium acherusicum (Costa, 1851)].

Photis brevipes Shoemaker, 1942

Previous record: Wakabara and Serejo 1998; ValérioBerardo and Miyagi 2000.

Exoedicerotidae Barnard and Drummond, 1982

Bathyporeiapus ruffoi Escofet, 1971

Previous record: Alves and Pezzuto 2009.

Phoxocephalopsidae Barnard and Drummond, 1982

Phoxocephalopsis zimmeri Schellenberg, 1931

Previous record: Alves and Pezzuto 2009.

Talitridae Rafinesque, 1815

Atlantorchestoidea brasiliensis (Dana, 1853)

Previous record: Alves and Pezzuto 2009.

Chelorchestia darwini (Müller, 1864)

Previous record: Wakabara and Serejo 1998 (cited as

Orchestia darwini Müller, 1864).

Talorchestia dentata (Filhol, 1885)

Previous record: Wakabara and Serejo 1998 (cited as

Orchestia tucurauna Müller, 1864)

Decapoda Latreille, 1801

Aeglidae Dana, 1852

Aegla brevipalma Bond-Buckup and Santos, 2012

Specimens in a collection: Rio Matador, Rio Uruguai basin, BR 282, km 136, Bom Retiro, 2749'02” S, 49³3'15” W, MZUSP 23465; UFRGS 3005; UFRGS 3010. Previous record: Santos et al. 2012.

Aegla camargoi Buckup and Rossi, 1977

Specimens in a collection: Rio Capivaras, Rio Pelotas basin, Bom Jardim da Serra, UFRGS 3834; Rio Cachoeira, Rio Pelotas basin, Jardim da Serra, UFRGS 3839; Rio Lajeadinho, Rio Pelotas basin, Jardim da Serra, UFRGS 3844; Rio Barrinha, Rio Pelotas basin, Jardim da Serra, UFRGS 3903.

Aegla franciscana Buckup and Rossi, 1977

Specimens in a collection: tributary of Rio Capivaras, Jardim da Serra, 28²5'28” S, 49³8'50” W, UFRGS 3841. Previous record: Bond-Buckup 2003.

Aegla jarai Bond-Buckup and Buckup, 1994

Specimens in a collection: Arroio Amola Faca, km 239, BR

116, Curitibanos, UFRGS 0248; Arroio Bandeirinhas, Lages UFRGS 0249H, 0249P, 0344; Rio Jacutinga, Concordia, UFRGS 0256; Rio Ponte Alta, Ponte Alta, UFRGS 0343; tributary of Rio Jacutinga, Arabutã, UFRGS 1117; tributary of Rio Matador, Bom Retiro, UFRGS 1290; Rio Bonito, BR 
282, Bocaina Sul, UFRGS 2054; Urubici, UFRGS 2056; Rio Espingarda, Indaial, UFRGS 2948; Rio Espingarda, National Park of the Serra do Itajaí, 2701'25.97" S, 4909'08.71" W, Rio Itajaí-Açú basin, Indaial, UFRGS 2991; waterfall Avencal, Ubirici, UFRGS 3003; Rio das Pedras, Santa Cecilia, 2707'28” S, 50²7'22”' W, UFRGS 3011; Rio Amola Faca, Lages, 2744'45" S, 50²0’04" W, UFRGS 3015; Rio Matador and tributary, BR 282, km 136, Bom Retiro, 2749'02" S, 49³3'15” W, UFRGS 3017; tributary of Rio Canoas, BR 282, between Lages and Bom Retiro, 27²7'10" S, 49³8'35" W, UFRGS 3020; Ribeirão Caeté, waterfall Ferdinand Schadrack, Blumenau, UFRGS 3203; Rio Águas Brancas, SC 430, tributary of Rio Canoas, Urubici, 2756'52" S, 49³4'38”' W, UFRGS 3720; Rio Barrinha, Rio Pelotas basin, Bom Jardim da Serra, 2820'48” S, 49³6'2.6” W, UFRGS 3836, 3902; Rio Baú, Rio Pelotas basin, Bom Jardim da Serra, 28 20'15" S, 49³7'47" W, UFRGS 3905; Rio Lajeadinho, Rio Pelotas basin, Bom Jardim da Serra, 28²7'1.5” S, 49³9'25.9” W, UFRGS 3908; Arroio Passo Fundo, Rio Caveira basin, Painel, 2754'34.4" S, 5005'14.6” W, UFRGS 3912; Rio Antoninha, Rio Lava-Tudo basin, São Joaquim, 28¹5'3.9" S, 4958'16" W, UFRGS 3922; Ponte Alta, Rio Canoas basin, Bocaina do Sul, 2744'4.4” S, 4959'1.0” W, UFRGS 3924; Rio Pessegueiro, Rio Canoas basin, Bocaína do Sul, $27^{\circ} 45^{\prime} 44^{\prime \prime}$ S, 5001'53,6” W, UFRGS 3925; tributary of Rio Pessegueiro, Rio Canoas basin, Bocaína do Sul, 2744'29" S, 5001'21.7" W, UFRGS 3926; Rio Pericó, Rio Pelotas basin, São Joaquim, 28¹1'58,5” S, 4945'54.9” W, UFRGS 3930; Rio Amola Faca, BR 116, Rio Caveiras basin, Lages, 274' 47.2" S, 50²0'5” W, UFRGS 3936; Rio Capoeiras, Rio Canoas basin, Urubici, $2^{\circ} 01^{\prime} 56.3^{\prime \prime} \mathrm{S}, 4^{\circ} 36^{\prime} 56.2^{\prime \prime} \mathrm{W}$, UFRGS 3937; Rio Ponte Alta , Rio Canoas basin, Ponte Alta, 27²8'46.4" S, 50²2'49.4" W, UFRGS 3940, 3941, 3942; Rio dos Cachorros, Rio Canoas basin, Ponte Alta, 27²1'38.3" S, 50²6'2.3” W, UFRGS 3943; Arroio between Curitibanos and Pouso Redondo, São Cristóvão do Sul, tributary of Rio Marombas, 27¹8'6.8” S, 50¹8'5.4" W, UFRGS 3947; Rio Águas Pretas, Rio Canoas basin,

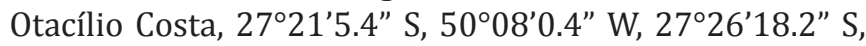
509'52.9" W, UFRGS 3952, 3953; Rio da Areia, Rio Canoas basin, Otacílio Costa, 27²7'52" S, 5007'20" W, UFRGS 3954; Rio dos Índios, Ribeirão das Palmeiras, Rio Canoas basin, Palmeiras, 27³4'15.5” S, 50¹0'6.7” W, UFRGS 3955; Arroio do Cerradinho, Rio Canoas basin, Palmeiras, 2735'16.8” S, 506'29.1” W), UFRGS 3956; Rio Camboim, Rio Canoas basin, Palmeiras, 27³5’35.3” S, 505'16.7” W, UFRGS 3957; Rio dos Macacos, Rio Canoas basin, Rio do Vale, 27²1'28.0" S, 50³'9.2" W, UFRGS 3958; Ribeirão do Cadeado, Rio Canoas basin, Casa Queimada, 2744'58.6” S, 50²'21.4" W, UFRGS 3959; Rio Amola Faca, Rio Caveiras basin, Lages, $27^{\circ} 45^{\prime} 41.7^{\prime \prime}$ S, 50²5' 5.2” W, UFRGS 3960; Rio Passo Fundo, Rio Caveiras basin, São José do Cerrito, 2743'45.6” S, 50³1'16.6” W, UFRGS 3961; Lajeado Refuga Baiano, Rio Caveiras basin, São José do Cerrito, 2741'7.8” S, 50³3'56.9” W, UFRGS 3962; Lajeado dos Motas, Rio Caveiras basin, São José do Cerrito, $27^{\circ} 48^{\prime} 14^{\prime \prime}$ S, 50²7'36.6” W, UFRGS 3963; Rio Caveiras basin, Capão Alto, 2755'50.5” S, 50³0'56.1" W, UFRGS 3964; tributary

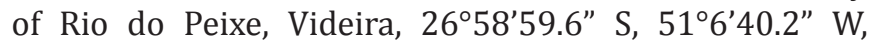
UFRGS 4004; Arroio das Antas, Otacílio Costa, UFRGS 4266. Previous record: Bond-Buckup 2003; Boos et al.
2006; Bond-Buckup et al. 2010.

\section{Aegla leachi Bond-Buckup and Santos, 2012}

Specimens in a collection: tributary of Rio Painel, Rio Uruguai basin, Painel, 2754'34.4" S, 5005'14.6” W, MZUSP 23466; UFRGS 3910P; Rio Galafre, Rio Uruguai basin, Urupema, 2752'58” S, 5002'10.6” W, UFRGS 3933; tributary of Rio Marombas, São Cristóvão do Sul, 27¹5'55" S, 50²8'14.1" W, UFRGS 3944; tributary of Rio Marombas, São Cristóvão do Sul, 27¹8'6.8” S, 50¹8'5.5” W, UFRGS 3950. Previous record: Santos et al. 2012.

\section{Aegla leptodactyla Bond-Buckup and Buckup, 1994}

Specimens in a collection: Rio Lajeadinho, Rio Pelotas basin, Bom Jardim da Serra, 28 $28^{\circ} 46^{\prime \prime}$ S, 4943'20.6” W, UFRGS 3845.

\section{Aegla marginata Bond-Buckup and Buckup, 1994}

Specimens in a collection: Rio Troncos, tributary of Rio da Prata, Joinville, UFRGS 1317; Afluente do Rio Lambedor, Chapecó, UFRGS 1344. Previous record: Bond-Buckup 2003.

\section{Aegla muelleri Bond-Buckup and Buckup, 2010}

Specimens in a collection: Rio Passa Quatro, Rio ItajaíAçú basin, Monte Castelo, 26²8'14" S, 50¹1'59” W, UFRGS 2979P, 2980P; Rio Espingarda, Rio Itajaí-Açú basin, Indaial, National Park of the Serra do Itajaí, $27^{\circ} 01^{\prime} 24^{\prime \prime}$ S, 4909'06" W, UFRGS 2982, 3385, 3570P, 3571, 4117H, 4117P. Previous record: Bond-Buckup et al. 2010.

\section{Aegla oblata Bond-Buckup and Santos, 2012}

Specimens in a collection: Rio Engenho Velho, Rio Uruguai basin, Painel, 2754'12.8” S, 5004'19.6” W, MZUSP 23467; UFRGS 3916P; UFRGS 3913; Rio Caronas, Rio Lava Tudo basin, Urupema, 2759'42.9” S, 4951'19.6" W, UFRGS3920; Rio Antoninha, Rio Lava Tudo basin, São Joaquim, 28¹5'3.9" S, 4958'16" W, UFRGS 3923; Rio Périco Redondo, Rio Pelotas basin, São Joaquim, 28¹1'58.5” S, 4945'54.9" W, UFRGS 3928; Rio Lava-Tudo, Rio Pelotas basin, Urubici, 28 05'17.9” S, 49³8'23.7” W, UFRGS 3935; Rio Lava-Tudo, Rio Pelotas basin, São Joaquim, 2809'05.3" S, 4943'04.8” W, UFRGS 3939. Previous record: Santos et al. 2012.

\section{Aegla odebrechtii Müller, 1876}

Specimens in a collection: Bom Jardim da Serra, UFRGS 2125; Rio Belchior, Gaspar, UFRGS 2390; Rio Itajai-Açu, Atalanta, UFRGS 2676; BR 282, tributary of Rio Canoas, between Lages and Bom Retiro 27 47'10" S, 49³8'35" W, UFRGS 3012; Rio Barrinha, Rio Pelotas basin, Bom Jardim da Serra, 28²0'48” S, 49³6'2.6” W, UFRGS 3835; Rio Cachoeira, Rio Pelotas basin, Bom Jardim da Serra, 28¹8'26" S, 49³7'1.4” W, UFRGS 3840; Rio Pelotas basin, Bom Jardim da Serra, 28¹6’19” S, 49³5’31.4” W, UFRGS 3907; Ribeirão Engenho Velho, Rio Caveiras basin, Painel, 2754'12.8”S, 504'19.6”W, UFRGS 3915; Rio Caronas, Rio Lava-Tudo basin, Urupema, 2759'42.9” S, 4951'19.6” W, UFRGS 3919; Rio do Tigre, Rio Canoas basin, 2753'54.3"S, 4944'59.8" W, UFRGS 3921; Rio Caçapava tributary of Rio Lava-tudo, Rio Canoas basin, São Joaquim, 28¹2'8.3" S, 4959'32"W, UFRGS 3927; Rio Galafre, Rio Caveiras basin, Urupema, 2752'58” S, 5002'10.6” W, UFRGS 3932; Rio Capoeiras, Rio Canoas basin, Urubici, 2752'58” S, 5002'10.6" W, UFRGS 3934; tributary of Rio Marombas, Rio Canoas basin, São Cristovão Sul, 27¹5'55" S, $50^{\circ} 28^{\prime} 14.1^{\prime \prime} \mathrm{W}, 27^{\circ} 16^{\prime} 18.6^{\prime \prime} \mathrm{S}, 50^{\circ} 30^{\prime} 13.3^{\prime \prime} \mathrm{W}, 27^{\circ} 18^{\prime} 6.8^{\prime \prime}$ S, 50¹8'5.5” W, UFRGS 3945, 3946, 3948, 3949. Previous 
record: Bond-Buckup 2003; Bond-Buckup et al. 2010. Aegla parana Schmitt, 1942

Specimens in a collection: Rio Timbó, São Pedro, UFRGS 1811. Previous record: Bond-Buckup 2003.

\section{Aegla parva Bond-Buckup and Buckup, 1994}

Specimens in a collection: tributary of Rio Cubatão, Águas Mornas, UFRGS 1295; tributary of Rio Pinheiros, Anitopolis, UFRGS 2057. Previous record: Bond-Buckup 2003.

\section{Aegla platensis Schmitt, 1942}

Specimens in a collection: Rio Lambedor, Chapecó, UFRGS 1105; Rio Rancho Grande, Peritiba, UFRGS 1115; Rio Marombas, Curitibanos, CCDB 2798. Previous record: Bond-Buckup 2003.

Aegla pomerana Bond-Buckup and Buckup, 2010

Specimens in a collection: tributary of Rio Itajaí-Açú, Pomerode, UFRGS 2679P, 2993P; Campo Alegre, Joinville, UFRGS 3262P. Previous record: Bond-Buckup et al. 2010. Aegla rossiana Bond-Buckup and Buckup, 1994

Specimens in a collection: Rio Jordão, Criciúma, UFRGS 00230H, 00230P, 232, 233, 243; tributary of Rio Jordão, Sideropolis, 28 $8^{\circ} 4^{\prime} 45^{\prime \prime}$ S, 49³0'20" W, UFRGS 3042; Ariribá, Itajaí, 2657’38.8” S, 48³9’15.4” W, CCDB 1938. Previous record: Bond-Buckup 2003.

\section{Aegla schmitti Hobbs III, 1979}

Specimens in a collection: Rio Negro basin, 26³9'37" S 50¹6'39” W, UFRGS 5346. Previous record: Bond-Buckup 2003.

\section{Aegla serrana Buckup and Rossi, 1977}

Specimens in a collection: Rio das Antas, Rio do Peixe basin, 265' 6.4" S, 513'45.2" W, UFRGS 4005.

Aegla singularis Ringuelet, 1948

Specimens in a collection: Rio das Antas, Rio do Peixe basin, 2654'6.4" S, 513'45.2” W, UFRGS 4006.

Aegla spinosa Bond-Buckup and Buckup, 1994

Specimens in a collection: Arroio São João, tributary of Rio Canoas, UFRGS 0246H, 0246P; Rio Jacutinga, Concordia, UFRGS 0251; Arroio Invernada, tributary of Rio Pelotas, UFRGS 1181; tributary of Rio Matador, Bom Retiro, UFRGS 1294; Urubici, SC, UFRGS 1421; headspring of the Rio Canoas, Serra Corvo Branco, UFRGS 2675; Rio Águas Brancas, tributary of Rio Canoas, Ubirici, 2756'52" S, 49³4'38" W, UFRGS 3009; Rio Baú, Rio Pelotas basin, Bom Jardim da Serra, 28 $20^{\prime} 15^{\prime \prime}$ S, 49³7'47" W, UFRGS 3837, 3838; Rio Capivaras, Rio Pelotas basin, Bom Jardim da Serra, 2824'20" S, 49³8'26" W, UFRGS 3842; Rio Cachoeira, Rio Pelotas basin, Bom Jardim da Serra, 28¹8'26" S, 49³7'1.4” W, UFRGS 3904; Rio Barrinha, Rio Pelotas basin, Bom Jardim da Serra, 28 20'48” S, 49³6'2.6” W, UFRGS 3906, 3911; Rio Barrinha, Rio Pelotas basin, Bom Jardim da Serra, 28²8'46” S, 4943'20.6” W, UFRGS 3909; Arroio Engenho Velho, Rio Caveiras basin, Painel, 2754'12.8” S, 5004' 19.6” W, UFRGS 3914; Rio Pericó, Rio Pelotas basin, São Joaquim, 2754'12.8” S, 5004'19.6” W, UFRGS 3929; Rio Galafre, Rio Caveiras basin, Urupema, 2752'58' S, 5002'10.6” W, UFRGS 3931; tributary of Rio Marombas, Rio Canoas basin, São Cristovão Sul, 27²1 6.8” S, 50¹8'5.5” W, UFRGS 3951; tributary of Rio Canoas, Campos Novos, 27³3'3.3" S, 5126'29.8” W, UFRGS 4003. Previous record: Bond-Buckup 2003.

\section{Hepatus gronovii Holthuis, 1959}

Specimens in a collection: Baía da Babitonga, CEPSUL 118; Baía da Babitonga, CEPSUL 119; $27^{\circ} 15.32^{\prime}$ S, 48²6.12’ W,

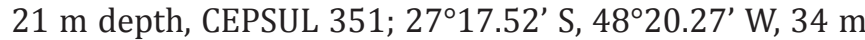
depth CEPSUL 468; $27^{\circ} 16.36^{\prime}$ S, 48¹7.71' W, 50 m depth CEPSUL 472. Previous record: Melo 1996.

Hepatus pudibundus (Herbst, 1785)

Specimens in a collection: $26^{\circ} 25.68^{\prime} \mathrm{S}, 48^{\circ} 22.95^{\prime} \mathrm{W}, 31$ 35 m depth, CEPSUL 112; 2625.68' S, 48²2.95’ W, 31-35 $m$ depth, CEPSUL $124 ; 2^{\circ} 25.68^{\prime} \mathrm{S}, 4^{\circ} 22.95^{\prime} \mathrm{W}, 31-35$ m depth, CEPSUL 125; 2708.62' S, 48²6.36’ W, $28 \mathrm{~m}$ depth, CEPSUL 248; shelf adjacent to Baía da Babitonga,

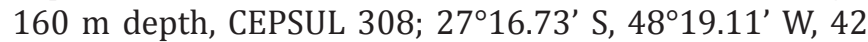
m depth, CEPSUL 349; 27⒖66’ S, 48²1.24' W, 38 m depth, CEPSUL 463; Praia dos Ingleses, Florianópolis, UFRGS 1847; Garopaba, UFRGS 1890; Ponta das Canas, Florianópolis, UFRGS 1891. Previous records: Melo 1996; 2010; Branco and Fracasso 2004; Bouzon and Freire 2007; Freitas Jr. et al. 2010.

Osachila antillensis Rathbun, 1916

Previous record: Melo 1996; 2010.

Osachila tuberosa Stimpson, 1871

Previous record: Melo 1996; 2010.

\section{Albuneidae Stimpson, 1858}

Albunea paretii Guérin-Menéville, 1853

Previous records: Calado 1999; Melo 1999.

Blepharipoda doelloi Schmitt, 1942

Previous record: Melo 1999.

\section{Lepidopa richmondi Benedict, 1903}

Specimens in a collection: Praia Brava, Florianópolis, UFRGS 2166; Praia de Bombas, Porto Belo, SC, UFRGS 3414, 3417; Praia dos Ingleses, Florianópolis, UFRGS 3603. Previous records: Calado 1999; Melo 1999; Alves and Pezzuto 2009.

Lepidopa venusta Stimpson, 1860

Previous records: Calado 1999; Melo 1999.

\section{Alpheidae Rafinesque, 1815}

\section{Alpheus angulosus McClure, 2002}

Previous record: Christoffersen 1998 (cited as Alpheus armillatus).

Alpheus bouvieri A. Milne-Edwards, 1878

Specimens in a collection: Praia de Cabeçudas, $26^{\circ} 55^{\prime} 46.2^{\prime \prime}$ S, 48³7'48.9" W, CCDB 1877; Praia dos Ingleses, 2708'39.7" S, 48²8'38.2" W, Bombinhas, CCDB 1886.

Alpheus floridanus Kingsley, 1878

Previous record: Christoffersen 1998.

Alpheus intrinsecus Bate, 1888

Previous record: Christoffersen 1998.

Alpheus nuttingi (Schmitt, 1924)

Specimens in a collection: Praia da Conceição, Bombinhas, $27^{\circ} 12^{\prime} 10.3^{\prime \prime} \mathrm{S}, 48^{\circ} 29^{\prime} 22.0^{\prime \prime} \mathrm{W}$, CCDB 1880. Previous record: Christoffersen 1998.

Alpheus pouang Christoffersen, 1979

Previous record: Christoffersen 1998.

Synalpheus apioceros Coutière, 1909

Previous record: Christoffersen 1998.

Synalpheus brevicarpus (Herrick, 1891)

Previous record: Christoffersen 1998. 
Synalpheus fritzmuelleri Coutière, 1909

Previous record: Christoffersen 1998.

Synalpheus latastei tenuispina Coutière, 1909

Previous record: Christoffersen 1998.

\section{Atyidae de Haan, 1849}

\section{Atya scabra (Leach, 1815)}

Specimens in a collection: $28^{\circ} 01^{\prime} 02^{\prime \prime}$ S, 48 $8^{\circ} 39^{\prime} 27^{\prime \prime} \mathrm{W}$, UFRGS 3185. Previous records: Ramos-Porto and Coelho 1998; Bond-Buckup and Buckup 1999; Melo 2003.

\section{Potimirim brasiliana Villalobos, 1959}

Specimens in a collection: Porto Belo, UFRGS 0650; tributary of Rio da Barra, Bombas, UFRGS 0651; Praia Brava, Florianópolis, UFRGS 2041; Praia da Vigia, Garopaba, UFRGS 2328, 2329, 2330, 2331, 2332. 2333, 2334, 2335, 2375, 3340, 3344; BR 376, Garuva, 2600'17.6”'S, 4852'17.1"W, CCDB 2116; Previous records: Ramos-Porto and Coelho 1998 (cited as P. glabra); Melo 2003 (cited as P. glabra); Torati and Mantelatto (2012).

\section{Potimirim potimirim Müller, 1881}

Specimens in a collection: Rio Cachoeira, Governador Celso Ramos, 27²5’08.6”S, 48³5'47.4”W, CCDB 1893; Blumenau, MZUSP 691. Previous records: Müller 1881; Ramos-Porto and Coelho 1998; Melo 2003; Torati and Mantelatto (2012).

\section{Belliidae Dana, 1852}

Corystoides chilensis Lucas, 1844

Previous record: Melo 1996; 2010.

\section{Calappidae De Haan, 1833}

\section{Acanthocarpus alexandri Stimpson, 1871}

Specimens in a collection: $26^{\circ} 13,22^{\prime} \mathrm{S}, 46^{\circ} 26,58^{\prime} \mathrm{W}, 210$ $\mathrm{m}$ depth, CEPSUL 190; 2648,79’ S, 4644,62' W, $208 \mathrm{~m}$ depth, CEPSUL 238; coast of Santa Catarina state, 20 - 100 $m$ depth, CEPSUL 399. Previous record: Melo 1996; 2010; Bernardes et al. 2005.

Callapa angusta A. Milne-Edwards, 1880

Previous record: Melo 1996.

Callapa galloides Stimpson, 1859

Previous records: Melo 1996 [cited as Callapa gallus (Herbst, 1803)]; Bouzon and Freire 2007 (cited as C. gallus).

\section{Callianassidae Dana, 1852}

\section{Callichirus major (Say, 1818)}

Specimens in a collection: Praia Barra do Saí, Itapoá, 2600'25.4" S, 48³6'23.45” W, CCDB 2184; Praia Central, Balneário Camboriú, 2659'26" S, 48³7'42" W, CCDB 2868. Previous record: Melo 1999.

Sergio guassutinga (Rodrigues, 1971)

Previous record: Melo 1999.

Sergio mirim (Rodrigues, 1971)

Specimens in a collection: Porto Belo, UFRGS 3397. Previous record: Melo 1999.

Chirostylidae Ortmann, 1892
Uroptychus nitidus (A. Milne-Edwards, 1880)

Specimens in a collection: MZUSP 13201. Previous record: Melo-Filho 1999; 2006; Nucci et al. 2004 (29¹4.675' S, 4750.669' W, $506 \mathrm{~m}$ depth).

\section{Cyclodorippidae Ortmann, 1892}

Clythrocerus granulatus (Rathbun, 1898)

Previous record: Melo 1996; Tavares 1996.

Deilocerus perpusillus (Rathbun, 1901)

Previous record: Melo 1996; 2010; Tavares 1996.

Cymonomidae Bouvier, 1898

Cymonomus quadratus A. Milne-Edwards, 1880

Previous record: Melo 1996; 2010.

\section{Diogenidae Ortmann, 1892}

\section{Calcinus tibicen (Herbst, 1791)}

Specimens in a collection: Praia dos Ingleses, Bombinhas, 2708'39.7" S, 48²8'38.2” W, CCDB 1884; Previous record: Rieger and Giraldi 1997a; Bouzon and Freire 2007.

Clibanarius antillensis Stimpson, 1859

Specimens in a collection: Praia de Cabeçudas, $26^{\circ} 55^{\prime} 46.2^{\prime \prime}$ S, 48³7'48.9” W, CCDB 1876. Previous record: Rieger 1997; Melo 1999.

Clibanarius sclopetarius (Herbst, 1796)

Specimens in a collection: Vigoreli, Joinville, UFRGS 4167; Tapera da Barra do Sul, Florianópolis, 27²7'24"S, 48³3'511" W, CCDB 2904. Previous record: Coelho and Ramos-Porto 1987; Rieger 1997; Melo 1999.

Clibanarius vittatus (Bosc, 1802)

Specimens in a collection: Praia do Sambaqui, Florianópolis, 27²9'12.7” S, 48³2'20.0” W, CCDB 1889; Barra da Lagoa, Florianópolis, 273' S, 48²5’ W, CCDB 2946. Previous record: Coelho and Ramos-Porto 1987; Rieger 1997; Melo 1999.

Dardanus insignis (de Saussure, 1858)

Specimens in a collection: $26^{\circ} 41.53^{\prime} \mathrm{S}, 47^{\circ} 43.85^{\prime} \mathrm{W}, 80 \mathrm{~m}$ depth, CEPSUL 23; 26³1.74' S, 48¹9.9' W, 40 m depth, CEPSUL 24; coast of Santa Catarina state, 38-41 m depth, CEPSUL 150. Previous record: Coelho and Ramos-Porto 1987; Rieger 1997; Melo 1999; Branco and Fracasso 2004; Bouzon and Freire 2007.

Isocheles sawayai Forest and Saint Laurent, 1968

Specimens in a collection: beach of Balneário Camboriú, UFRGS 1910; 26¹3' S, 48³0’ W, CCDB 3054. Previous record: Coelho and Ramos-Porto 1987; Rieger 1997; Melo 1999.

Loxopagurus loxochelis (Moreira, 1901)

Specimens in a collection: $26^{\circ} 27.62^{\prime} \mathrm{S}, 48^{\circ} 30.87^{\prime} \mathrm{W}, 20 \mathrm{~m}$ depth, CEPSUL $33 ; 6^{\circ} 25.95^{\prime}$ S, $48^{\circ} 34.55^{\prime} \mathrm{W}, 12 \mathrm{~m}$ depth, CEPSUL 35; 2613' S, 48³0' W, CCDB 3053. Previous record: Coelho and Ramos-Porto 1987; Rieger 1997; Melo 1999; Branco and Fracasso 2004; Freitas Jr. et al. 2010

Paguristes pauciparus Forest and Saint Laurent, 1968 Previous record: Rieger 1997; Melo 1999.

Paguristes robustus Forest and Saint Laurent, 1968 Previous record: Bouzon and Freire 2007.

Paguristes rostralis Forest and Saint Laurent, 1968 
Previous record: Coelho and Ramos-Porto 1987.

Paguristes tortugae Schmitt, 1933

Specimens in a collection: Florianópolis, CCDB 2512. Previous record: Rieger and Giraldi 1997a.

Petrochirus diogenes (Linnaeus, 1758)

Specimens in a collection: $26^{\circ} 17.72^{\prime} \mathrm{S}, 48^{\circ} 11.09^{\prime} \mathrm{W}, 40 \mathrm{~m}$ depth, CEPSUL 44; $26^{\circ} 29.74^{\prime} \mathrm{S}$, 48 $18.99^{\prime} \mathrm{W}, 40 \mathrm{~m}$ depth, CEPSUL 54; $26^{\circ} 24.62^{\prime} \mathrm{S}, 47^{\circ} 52.45^{\prime} \mathrm{W}, 60 \mathrm{~m}$ depth, CEPSUL 57; 26³1.74' S, 48¹9.9' W, 40 m depth, CEPSUL 65; Caixa D'Aço, Porto Belo, UFRGS 1375. Previous record: Coelho and Ramos-Porto 1987; Rieger 1997; Melo 1999.

\section{Dromiidae De Haan, 1833}

\section{Dromia erythropus (George Edwards, 1771)}

Specimens in a collection: Ganchos de Fora, Governador Celso Ramos, MZUSP 6182. Previous record: Melo and Campos Jr. 1999.

\section{Hypoconcha arcuata Stimpson, 1858}

Specimens in a collection: $26^{\circ} 31.41^{\prime}$ S, 48 $19.78^{\prime} \mathrm{W}, 40$ $\mathrm{m}$ depth, CEPSUL 18; Enseada de Balneário Camboriú, 2659'07" S, 48³5'58”W, FURG 3253. Previous record: Machado et al. 2009.

\section{Hypoconcha parasitica (Linnaeus, 1763)}

Previous records: Melo and Campos Jr. 1999; Bouzon and Freire 2007.

\section{Moreiradromia antillensis (Stimpson, 1858)}

Specimens in a collection: $26^{\circ} 17.09^{\prime} \mathrm{S}, 48^{\circ} 10.67^{\prime} \mathrm{W}, 40 \mathrm{~m}$ depth, CEPSUL 52. Previous records: Melo 1996; 2010; Melo and Campos Jr. 1999; Guinot and Tavares 2003.

\section{Epialtidae MacLeay, 1838}

Apiomithrax violaceus (A. Milne-Edwards, 1868)

Previous record: Melo 1996, 2010.

Epialtus bituberculatus H. Milne Edwards, 1834

Specimens in a collection: Santa Marta Grande Cape, Laguna, CEPSUL 126; Praia do Sambaqui, Florianópolis, 27²9'12.7” S, 48³2'20.0” W, CCDB 1887. Previous record: Bouzon and Freire 2007.

\section{Galatheidae Samouelle, 1819}

\section{Agononida longipes (A. Milne-Edwards, 1880)}

Specimens in a collection: $27^{\circ} 20^{\prime} \mathrm{S}, 47^{\circ} 04^{\prime} \mathrm{W}, 400 \mathrm{~m}$ depth, MZUSP 15355; $27^{\circ} 20^{\prime} \mathrm{S}, 4^{\circ} 13^{\prime} \mathrm{W}, 197 \mathrm{~m}$ depth, MZUSP 15367; $27^{\circ} 20^{\prime} \mathrm{S}, 4^{\circ} 06^{\prime} \mathrm{W}, 295 \mathrm{~m}$ depth, MZUSP 15349; $27^{\circ} 19^{\prime} \mathrm{S}, 47^{\circ} 12^{\prime} \mathrm{W}, 199 \mathrm{~m}$ depth, MZUSP 15361; 27ำ' $15^{\prime} \mathrm{S}$,

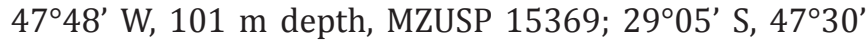
W, 250 m depth, FURG 393. Previous records: Melo 1999; Melo-Filho 1999; 2006; 2008; Melo-Filho and Melo 2001 (cited as Munida longipes).

\section{Munida constricta A. Milne-Edwards, 1880}

Specimens in a collection: $28^{\circ} 50^{\prime} \mathrm{S}, 47^{\circ} 35^{\prime} \mathrm{W}, 586 \mathrm{~m}$ depth,

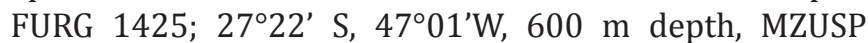
16316. Previous records: Melo 1999; Melo-Filho 1999; 2006; 2008; Melo-Filho and Melo 2001.

Munida flinti Benedict, 1902

Specimens in a collection: $26^{\circ} 13.22^{\prime} \mathrm{S}, 46^{\circ} 26.58^{\prime} \mathrm{W}, 210 \mathrm{~m}$ depth, CEPSUL 192; $26^{\circ} 48.79^{\prime} \mathrm{S}, 46^{\circ} 44.62^{\prime} \mathrm{W}, 208 \mathrm{~m}$ depth, CEPSUL 241; 264' S, 46²8' W, 390 m depth, MZUSP 10305, 10307, 15353. Previous records: Melo 1999; Melo-
Filho 1999; 2006; 2008; Melo-Filho and Melo 2001.

\section{Munida forceps A. Milne-Edwards, 1880}

Specimens in a collection: $26^{\circ} 13.22^{\prime} \mathrm{S}, 4^{\circ} 26.58^{\prime} \mathrm{W}, 210$ m depth, CEPSUL 193; 2648.72' S, 4644.99' W, 206 m depth, CEPSUL 194; 2648.79' S, 46²4.62' W, 208 m depth, CEPSUL 239; MZUSP 5173; FURG 756, 758. Previous records: Melo 1999; Melo-Filho 1999; 2006; Melo-Filho and Melo 2001.

Munida iris A. Milne-Edwards, 1880.

Specimens in a collection: $27^{\circ} 20^{\prime} \mathrm{S}, 4^{\circ} 06^{\prime} \mathrm{W}, 295 \mathrm{~m}$ depth, MZUSP 15350. Previous record: Melo-Filho 2006; 2008.

Munida irrasa A. Milne-Edwards, 1880

Previous record: Melo-Filho 1999; 2006.

Munidopsis polita (S. I. Smith, 1883)

Specimens in a collection: $27^{\circ} 21^{\prime} \mathrm{S}, 47^{\circ} 01^{\prime} \mathrm{W}, 598 \mathrm{~m}$ depth, MZUSP 16439. Previous record: Melo-Filho 2008.

\section{Gecarcinidae MacLeay, 1838}

Cardisoma guanhumi Latreille, 1828

Previous record: Branco 1991; Tavares 2002c.

\section{Geryonidae Colosi, 1923}

Chaceon ramosae Manning, Tavares and Albuquerque, 1989

Specimens in a collection: $29^{\circ} 03.95^{\prime} \mathrm{S}, 47^{\circ} 45.37^{\prime} \mathrm{W}, 600$

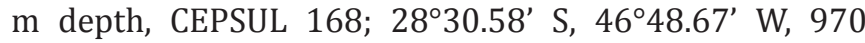

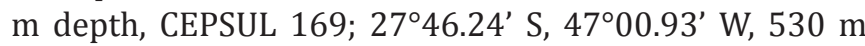
depth, CEPSUL 170; $27^{\circ} 41.24^{\prime} \mathrm{S}, 46^{\circ} 53.36^{\prime} \mathrm{W}, 772 \mathrm{~m}$

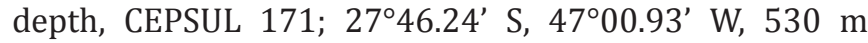
depth, CEPSUL 172; $29^{\circ} 05.96^{\prime} \mathrm{S}, 4^{\circ} 45.37^{\prime} \mathrm{W}, 560 \mathrm{~m}$ depth, CEPSUL 173; 2736.38' S, 4708.97' W, $468 \mathrm{~m}$ depth, CEPSUL 174; $26^{\circ} 50.94^{\prime} \mathrm{S}, 46^{\circ} 10.48^{\prime} \mathrm{W}, 620 \mathrm{~m}$ depth, CEPSUL 175; $27^{\circ} 41.11^{\prime} \mathrm{S}, 47^{\circ} 06.41^{\prime} \mathrm{W}, 530 \mathrm{~m}$ depth, CEPSUL 177; $26^{\circ} 18.23^{\prime} \mathrm{S}, 45^{\circ} 41.09^{\prime} \mathrm{W}, 750 \mathrm{~m}$ depth, CEPSUL 178; $27^{\circ} 46.24^{\prime} \mathrm{S}, 4^{\circ} 00.99^{\prime} \mathrm{W}, 530 \mathrm{~m}$

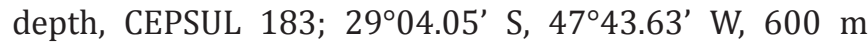
depth, CEPSUL 186; $27^{\circ} 05^{\prime} 14.26^{\prime \prime}$, $46^{\circ} 23^{\prime} 48.59^{\prime \prime} \mathrm{W}$, CCDB 2590. Previous record: Athiê and Rossi-Wongtschowski, 2004; Tavares and Pinheiro 2011.

\section{Goneplacidae MacLeay, 1838}

\section{Chasmocarcinus typicus Rathbun, 1898}

Specimens in a collection: $26^{\circ} 30.93^{\prime} \mathrm{S}, 4^{\circ} 26.3^{\prime} \mathrm{W}, 100 \mathrm{~m}$ depth, CEPSUL 14. Previous record: Melo 1996; 2010.

Eucratopsis crassimanus (Dana, 1852)

Previous record: Melo 1996.

Euryplax nitida Stimpson, 1859

Previous records: Melo 1996.

Frevillea hirsuta (Borradaile, 1916)

Specimens in a collection: $26^{\circ} 19.97^{\prime}$ S, $47^{\circ} 19.26^{\prime} \mathrm{W}, 100 \mathrm{~m}$ depth, CEPSUL 63. Previous record: Melo 1996; 2010.

Neopilumnoplax americana (Rathbun, 1898)

Previous record: Bouzon and Freire 2007.

Pseudorhombila octodentata Rathbun, 1906

Specimens in a collection: $26^{\circ} 41.53^{\prime} \mathrm{S}, 47^{\circ} 43.85^{\prime} \mathrm{W}, 80 \mathrm{~m}$ depth, CEPSUL 60. Previous record: Melo 1996; 2010.

Grapsidae MacLeay, 1838 
Cyrtograpsus affinis (Dana, 1851)

Previous record: Melo 1996.

Cyrtograpsus angulatus Dana, 1851

Specimens in a collection: Laguna, CEPSUL 120; Rio da Praia Brava, Itajaí, 2656’25.2” S, 48³7’42.8” W, CCDB 1902. Previous record: Melo 1996.

Geograpsus lividus (H. Milne Edwards, 1837)

Specimens in a collection: Costão do Santinho, Florianópolis, UFRGS 3790; 28³0'13.94" S, 4846'02.99" W, UFRGS 4771; Praia de Cabeçudas, Itajaí, 2655'46.2” S, 48³7’48.9” W, CCDB 1873. Previous record: Melo 1996.

Goniopsis cruentata (Latreille, 1803)

Specimens in a collection: Praia Brava, Florianópolis, UFRGS 2169; 28²8'03.60" S, 4851'43.31" W, UFRGS 4763; 2656’25.2” S, 48³7’42.8” W, Praia Brava, Itajaí, CCDB 1879. Previous records: Branco 1991; Melo 1996.

Neohelice granulata (Dana, 1851)

Specimens in a collection: Praia Brava, Florianópolis, UFRGS 1860; Florianópolis, UFRGS 1920; 28²8'03.60" S, 48 51'43.31" W, UFRGS 4756; 28 25'04.82" S, 48 $51^{\circ} 31.71^{\prime \prime}$ W, UFRGS 4761; 28²7'51.57" S, 48 $50^{\prime} 25.18^{\prime \prime}$ W, UFRGS 4769; Itajaí, MZUSP 670. Previous records: Melo 1990; 1996, Branco 1991; Branco et al. 2011 (cited as Chasmagnathus granulata).

Pachygrapsus gracilis (Saussure, 1858)

Specimens in a collection: $28^{\circ} 30^{\prime} 13.94^{\prime \prime}$ S, 4846'02.99” W, UFRGS 4764. Previous record: Melo 1996.

Pachygrapsus transversus (Gibbes, 1850)

Specimens in a collection: Armação de Itapocorói, Penha, CEPSUL 372; Ponta das Canas, Florianópolis, UFRGS 1854; Canasvieiras, Florianópolis, UFRGS 1868; Praia Brava, Florianópolis, UFRGS 3391, 4556; Praia Brava, Itajaí, 2657'34.0" S, 48³7'43.4” W, CCDB 1885; Praia de Cabeçudas, Itajaí, 2655'46.2" S, 48³7'48.9” W, CCDB 1940. Previous records: Melo 1996; Bouzon and Freire 2007.

\section{Planes cyaneus Dana, 1851}

Specimens in a collection: epibiont associated with sea turtles, CEPSUL 373; Praia Brava, Florianópolis, UFRGS 3389. Previous record: Melo 1996.

\section{Hippidae Latreille, 1825}

\section{Emerita brasiliensis Schmitt, 1935}

Specimens in a collection: Florianópolis, Praia Brava, UFRGS 1904; Praia Brava, Florianópolis, UFRGS 1991, 2167, 3352, 3394, 3408, 3416; Garopaba, UFRGS 3463. Previous records: Calado 1999; Melo 1999; Alves and Pezzuto 2009.

\section{Hippolytidae Dana, 1852}

\section{Exhippolysmata oplophoroides (Holthuis, 1948)}

Specimens in a collection: $26^{\circ} 13.5^{\prime} \mathrm{S}, 48^{\circ} 29.4^{\prime} \mathrm{W}, 15 \mathrm{~m}$ depth, CEPSUL 30, 49. Previous record: Christoffersen 1998; Bond-Buckup and Buckup 1999; Tavares 2002b; Branco and Fracasso 2004.

\section{Hippolyte obliquimanus Dana, 1852}

Specimens in a collection: Porto Belo, UFRGS 2371; Bombinhas, 2712'10.3” S, 48²9'22.0” W, CCDB 1874; Praia dos Ingleses, Bombinhas, $27^{\circ} 08^{\prime} 39.7^{\prime \prime} \mathrm{S}, 48^{\circ} 28^{\prime} 38.2^{\prime \prime}$ W, CCDB 1881; Florianópolis, 27²9’12.7” S, 48³2’20.0” W,
CCDB 1875; Praia do Sambaqui, Florianópolis, 27²9’12.7” S, 48³2'20.0” W, CCDB 2620, 2891, 2958. Previous record: Christoffersen 1998 (cited as H. curacaoensis Schmitt, 1924); Terossi and Mantelatto 2012.

Latreutes parvulus (Stimpson, 1866)

Specimens in a collection: Ponta das Canas, Florianópolis, UFRGS 0682.

Lysmata moorei (Rathbun, 1902)

Previous record: Christoffersen 1998.

Lysmata wurdemanni (Gibbes, 1850)

Previous record: Bond-Buckup and Buckup 1999.

\section{Homolidae De Haan, 1839}

Homola barbata (Fabricius, 1793)

Previous record: Melo 1996.

Inachidae MacLeay, 1838

Anisonotus atlanticus (Coelho, 1997)

Previous record: Coelho 2006.

Coryrhynchus algicola Stebbing, 1914

Previous record: Melo 1996; Coelho 2006; Bouzon and Freire 2007.

Ericerodes gracilipes (Stimpon, 1871)

Previous records: Melo 1996; Coelho 2006.

Eurypodius latreillii Guérin, 1825

Previous record: Melo 2010.

Podochela atlantica (Coelho, 1997)

Previous record: Melo 2010.

Stenorhynchus seticornis (Herbst, 1788)

Specimens in a collection: Praia da Conceição, Bombinhas, 27²'10.3" S, 48²9'22.0” W, CCDB 1872. Previous records: Melo 1996; 2010; Bouzon and Freire 2007; Almeida and Coelho 2008.

\section{Inachoididae Dana, 1851}

Collodes rostratus A. Milne-Edwards, 1879

Previous records: Melo 1996; 2010; Almeida and Coelho 2008.

Euprognatha acuta A. Milne-Edwards, 1880

Previous record: Melo 1996.

Euprognatha gracilipes A. Milne-Edwards, 1878

Previous record: Melo 1996.

Euprognatha rastellifera Stimpson, 1871

Previous record: Melo 2010.

Inachoides forceps A. Milne-Edwards, 1879

Specimens in a collection: MZUSP 12609, 6067. Previous record: Santana and Tavares 2009.

Leurocyclus tuberculosus (H. Milne Edwards and Lucas, 1843)

Specimens in a collection: $26^{\circ} 24.74^{\prime} \mathrm{S}, 4^{\circ} 52.5^{\prime} \mathrm{W}, 60 \mathrm{~m}$ depth, CEPSUL 42; $26^{\circ} 40.22^{\prime} \mathrm{S}, 4^{\circ} 43.06^{\prime} \mathrm{W}, 81 \mathrm{~m}$ depth, CEPSUL 46; $26^{\circ} 41.53^{\prime} \mathrm{S}, 47^{\circ} 43.89^{\prime} \mathrm{W}, 80 \mathrm{~m}$ depth, CEPSUL 47; CEPSUL 450; CEPSUL 469; 2709.67' S, 49¹6,91' W, $49 \mathrm{~m}$ depth, CEPSUL 473. Previous record: Melo 1996; 2010.

Pyromaia tuberculata (Lockington, 1877)

Specimens in a collection: Enseada de Balneário Camboriú, 2659'07” S, 48³5'58” W, FURG 3254. Previous record: Machado et al. 2009. 
Latreilliidae Stimpson, 1858

Latreillia williamsi Melo, 1990

Previous record: Melo 1996, 2010

\section{Leucosiidae Samouelle, 1819}

\section{Myropsis quinquespinosa Stimpson, 1871}

Specimens in a collection: $26^{\circ} 48.72^{\prime} \mathrm{S}, 46^{\circ} 44.99^{\prime} \mathrm{W}, 206 \mathrm{~m}$ depth, CEPSUL 195; shelf adjacent to Baía da Babitonga, 90-330 m depth, CEPSUL 305. Previous records: Melo 1996; 2010; Bernardes et al. 2005.

Persephona crinita Rathbun, 1931

Previous record: Melo 1996.

Persephona lichtensteinii Leach, 1817

Specimens in a collection: $26^{\circ} 13.5^{\prime} \mathrm{S}, 48^{\circ} 29.4^{\prime} \mathrm{W}, 15 \mathrm{~m}$ depth, CEPSUL 62; $27^{\circ} 08.62^{\prime} \mathrm{S}, 48^{\circ} 26.36^{\prime} \mathrm{W}, 28 \mathrm{~m}$ depth, CEPSUL 247; $27^{\circ} 16.8^{\prime}$ S, $48^{\circ} 17.96^{\prime}$ W, 49 m depth, CEPSUL 347. Previous record: Branco and Fracasso 2004; Freitas Jr. et al. 2010.

\section{Persephona mediterranea (Herbst, 1794)}

Specimens in a collection: $26^{\circ} 25.68^{\prime} \mathrm{S}, 4^{\circ} 22.5^{\prime} \mathrm{W}, 31-$ $35 \mathrm{~m}$ depth, CEPSUL 113; 26³0.27' S, 48¹9.19' W, $41 \mathrm{~m}$ depth, CEPSUL 240; Barra do Sul, CEPSUL 362; $7^{\circ} 16.13^{\prime}$ $\mathrm{S}, 48^{\circ} 27.892^{\prime} \mathrm{W}, 18 \mathrm{~m}$ depth, CEPSUL 475; 260.6' $56^{\prime \prime} \mathrm{S}$, 48³1'11" W, CCDB 3049. Previous record: Melo 1996; 2010; Branco and Fracasso 2004.

Persephona punctata (Linnaeus, 1758)

Specimens in a collection: $26^{\circ} 13.53^{\prime} \mathrm{S}, 4^{\circ} 29.31^{\prime} \mathrm{W}, 18$ $\mathrm{m}$ depth, CEPSUL 50; Baía da Babitonga, CEPSUL 115; $27^{\circ} 08.36^{\prime} \mathrm{S}, 48^{\circ} 26.24^{\prime} \mathrm{W}, 28 \mathrm{~m}$ depth, CEPSUL 350; cost north of Santa Catarina, 20-100 m depth, CEPSUL 400; Ponta das Canas, Florianópolis, UFRGS 1934; 2606'56" S, 48³1'11" W, CCDB 3050. Previous records: Melo 1996; Branco and Fracasso 2004; Freitas Jr. et al. 2010.

\section{Luciferidae de Haan, 1849}

\section{Lucifer faxoni Borradaile, 1915}

Previous record: D'Incao 1998; 1999; Resgala Jr. et al. 2008.

Lucifer typus H. Milne Edwards, 1837

Previous record: D’Incao 1998.

Majidae Samouelle, 1819

\section{Eurypodius latreillei Guérin, 1828}

Previous record: Melo 1996.

Macrocoeloma captocerum (Stimpson, 1871)

Previous record: Bouzon and Freire 2007.

Pitho Iherminieri (Schramm, 1867)

Previous record: Bouzon and Freire 2007.

\section{Menippidae Ortmann, 1893}

\section{Eriphia gonagra (Fabricius, 1781)}

Specimens in a collection: Praia da Ribanceira, Imbituba, CEPSUL 371; Praia Brava, Florianópolis, UFRGS 3432; Praia Brava, Itajaí, 2657'34.0” S, 48³7'43.4” W, CCDB 1896. Previous records: Karasawa and Schweitzer 2006; Almeida and Coelho 2008.

Menippe nodifrons Stimpson, 1859
Specimens in a collection: Enseada de Itapocorói, Penha, CEPSUL 117; Garopaba, UFRGS 0022, 0023; Porto Belo, UFRGS 0133, 0168, 0547; Laguna, UFRGS 0548; Praia de Jurerê, Florianópolis, UFRGS 1867. Previous records: Melo 1996; Branco and Fracasso 2004; Bouzon and Freire 2007.

\section{Mithracidae Balss, 1929}

Microphrys bicornutus (Latreille, 1825)

Previous record: Melo 1996; 2010; Almeida and Coelho 2008.

Mithraculus forceps (A. Milne-Edwards, 1875)

Previous record: Rieger and Giraldi 1996; Bouzon and Freire 2007; Almeida and Coelho 2008.

Mithrax hispidus (Herbst, 1790)

Specimens in a collection: Ilha do Amendoim, Porto Belo, UFRGS 0172; Praia da Conceição, Bombinhas, 27¹1'55.02" S, 48²8'59.77" W, CCDB 1862. Previous records: Rieger and Giraldi 2001; Almeida and Coelho 2008.

Mithrax tortugae Rathbun, 1920

Specimens in a collection: Ilha do Amendoim, Porto Belo, UFRGS 0158. Previous records: Rieger and Giraldi 2001; Bouzon and Freire 2007.

Stenocionops furcatus (Olivier, 1791)

Previous records: Melo 1996; 2010; Almeida and Coelho 2008.

Stenocionops spinosissimus (de Saussure, 1857)

Specimens in a collection: $26^{\circ} 44.12^{\prime} \mathrm{S}, 4^{\circ} 32.27^{\prime} \mathrm{W}, 100$ $m$ depth, CEPSUL 134. Previous record: Melo 1996; 2010.

\section{Nephropidae Dana, 1852}

\section{Metanephrops rubellus (Moreira, 1903)}

Specimens in a collection: $26^{\circ} 31.52^{\prime}$ S, 47²6.51' W, $100 \mathrm{~m}$ depth, CEPSUL 13. Previous records: D'Incao 1999; Melo 1999.

Nephropsis aculeata Smith, 1881

Specimens in a collection: $25^{\circ} 58^{\prime} \mathrm{S}, 46^{\circ} 07^{\prime} \mathrm{W}, 268 \mathrm{~m}$ depth,

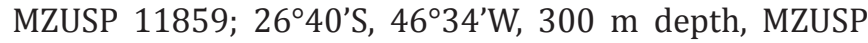
15186; $28^{\circ} 00.22^{\prime} \mathrm{S}, 46^{\circ} 52.38^{\prime} \mathrm{W}, 603 \mathrm{~m}$ depth, CEPSUL 391. Previous record: Dall'Occo et al. 2007.

Nephropsis rosea Bate, 1888

Specimens in a collection: $27^{\circ} 22^{\prime} \mathrm{S}, 47^{\circ} 01^{\prime} \mathrm{W}, 600 \mathrm{~m}$ depth, MZUSP 15250. Previous record: Dall'Occo et al. 2007.

\section{Ocypodidae Rafinesque, 1815}

\section{Ocypode quadrata (Fabricius, 1787)}

Specimens in a collection: Praia Brava, Florianópolis, UFRGS 1859, 3422, 3437. Previous record: Melo 1996; Alves and Pezzuto 2009.

\section{Uca burgesi Holthuis, 1967}

Specimens in a collection: Vigoreli, Joinvile, UFRGS 4165; Tapera da Barra do Sul, Florianópolis, 2747'14" S, 48³3'51.1"W, CCDB 1897; Barra do Saí, Itapoá, $26^{\circ} 00^{\prime} 25.4^{\prime \prime}$ S, 48³6'23.5” W, CCDB 2282.

\section{Uca leptodactyla Rathbun, 1898}

Specimens in a collection: Florianópolis, UFRGS 1933; Praia Brava, Florianópolis, UFRGS 2165. Previous record: Melo 1996.

Uca maracoani (Latreille, 1802-1803)

Previous record: Branco 1991. 


\section{Uca mordax (Smith, 1870)}

Specimens in a collection: Barra do Saí, Rio Saí Mirim, Itapoá, UFRGS 3679; Baía Babitonga, UFRGS 4022; Rio Cachoeira, Praia de São Miguel, Governador Celso Ramos, 27²5'18.9” S, 48³7'25.8” W, CCDB 1898; Rio Camarão, Praia de São Miguel, Governador Celso Ramos, 27²4’05.9” S, 48³7’06.9” W, CCDB 1900.

Uca rapax (Smith, 1870)

Specimens in a collection: Barra do Saí, Rio Saí Mirim, Itapoá, UFRGS 3679; Vigoreli, Joinville, UFRGS 4166. Previous record: Melo 1996.

\section{Uca thayeri Rathbun, 1900}

Specimens in a collection: Praia Ubatuba, São Francisco do Sul, UFRGS 0120; Florianópolis, UFRGS 2287; Praia Brava, Florianópolis, UFRGS 3434; Ponta das Canas, Florianópolis, UFRGS 3652. Previous records: Branco 1991; Melo 1996; Branco et al. 2011.

\section{Uca uruguayensis Nobili, 1901}

Specimens in a collection: estuary of Rio Itajaí-Açú, CEPSUL 374; Praia Ubatuba, São Francisco do Sul, UFRGS 0121; Praia da Mutuca, Florianópolis, 2741'49.4” S, 48³3'11.0" W, CCDB 1899; Itajaí, MZUSP 668. Previous records: Melo 1990; Branco 1991; Melo 1996; Branco et al. 2011.

Uca vocator (Herbst, 1804)

Previous record: Melo 1996

Ucides cordatus (Linnaeus, 1763)

Specimens in a collection: Meia Praia, Itapema, CEPSUL 122; Foz do Rio Camboriú, Balneário Camboriú, CEPSUL 311; Praia Brava, Florianópolis, UFRGS 2168; Baia da Babitonga, UFRGS 4024. Previous records: Branco 1991; Melo 1996; Tavares 2002c; Branco et al. 2011.

\section{Ogyrididae Hay and Shore, 1918}

Ogyrides alphaerostris (Kingsley, 1880)

Previous record: Christoffersen 1998.

\section{Paguridae Latreille, 1802}

\section{Pagurus brevidactylus (Stimpson, 1859)}

Specimens in a collection: $27^{\circ} 12^{\prime} 10.3^{\prime \prime} \mathrm{S}, 48^{\circ} 29^{\prime} 22.0^{\prime \prime} \mathrm{W}$, Praia da Conceição, Bombinhas, CCDB 1878. Previous record: Rieger 1997; Rieger and Giraldi 1997b; Melo 1999.

Pagurus criniticornis (Dana, 1852)

Specimens in a collection: $27^{\circ} 29^{\prime} 12.7^{\prime \prime}$ S, 48 $32^{\prime} 20^{\prime \prime} \mathrm{W}$, Praia do Sambaqui, Florianópolis, CCDB 1892. Previous record: Rieger 1997; Melo 1999.

Pagurus exilis (Benedict, 1892)

Previous record: Rieger 1997; Melo 1999.

Pagurus leptonyx Forest and Saint Laurent, 1968

Previous record: Rieger 1997; Melo 1999.

Pagurus provenzanoi Forest and Saint Laurent, 1968

Previous record: Rieger 1997; Melo 1999.

Rhodochirus rosaceus (A. Milne-Edwards and Bouvier, 1893)

Previous record: Rieger 1997; Melo 1999; Bouzon and Freire 2007.

\section{Palaemonidae Rafinesque, 1815}

\section{Leander paulensis Ortmann, 1897}

Specimens in a collection: Enseada de Balneário Camboriú,
2659'07” S, 48³5'58” W, FURG 3257. Previous record: Machado et al. 2010.

Macrobrachium acanthurus (Wiegmann, 1836)

Specimens in a collection: Rio Saí-Guaçu, Garuva, UFRGS 0697; Rio Itajaí (artificial lake), Blumenau, UFRGS 3292; São José Acaraí, São Francisco do Sul, UFRGS 3345; Barra do Saí, Rio Saí Mirim, Itapoá, UFRGS 3680; 4850'25.18”W - 28²7'51.57"S, UFRGS 4765; Rio da Praia de Bombas, Bombinhas, 2708'02.9” S, 48³0'49.1" W, CCDB 1915; Palmas, Florianópolis, 2720'27.6” S, 48³2'35.2” W, CCDB 1916; Rio da Praia Brava, Itajaí, 2656'25.2" S, 48³7'42.8” W, CCDB 1917; Fazenda Armação, Tijucas, 27²2’07.3" S, 48³3'36.2" W, CCDB 1918; Rio da Praia do Estaleiro, Camboriú, CCDB 1919; Rio Saí Mirim, Itapoá, 2600’25.3" S, 48³6'23.5” W, CCDB 2274; Rio Saí Mirim, 2601’35.8” S, 48³7'47.5" W, CCDB 2275; Rio Saí Mirim, Itapoá, 2559'23.9" S, 48³6'17.9" W, CCDB 2467; Praia Brava, Itajaí, 2657'38.8” S, 48³9'15.5” W, CCDB 2506. Previous records: Bond-Buckup and Buckup 1989; Müller et al. 1993; Ramos-Porto and Coelho 1998; Bond-Buckup and Buckup 1999; Melo 2003; Branco et al. 2011.

Macrobrachium carcinus (Linnaeus, 1758)

Specimens in a collection: Porto Belo, UFRGS 645; Rancho da Amizade, Florianópolis, UFRGS 884. Previous records: Bond-Buckup and Buckup 1989; Ramos-Porto and Coelho 1998; Bond-Buckup and Buckup 1999; Melo 2003.

Macrobrachium heterochirus (Wiegmann, 1836)

Specimens in a collection: Praia Brava, Florianópolis, UFRGS 2040; Rio Amola Faca, Araranguá, UFRGS 2118. Previous records: Bond-Buckup and Buckup 1989; RamosPorto and Coelho 1998; Bond-Buckup and Buckup 1999; Melo 2003.

Macrobrachium jelskii (Miers, 1877)

Previous records: Ramos-Porto and Coelho 1998; Melo 2003. Remarks: Voucher material no specified; occurrence in the state is doubtful.

Macrobrachium nattereri (Heller, 1862)

Previous record: Melo 2003. Remarks: Voucher material no specified; occurrence in the state is doubtful.

Macrobrachium olfersi (Wiegmann, 1836)

Specimens in a collection: Ponta das Canas, Florianópolis, UFRGS 0710; Garopaba, UFRGS 2069; Garopaba, UFRGS 2071; Laguna, UFRGS 2353; Garopaba, UFRGS 2354, Garopaba, UFRGS 2355; Rio da Praia do Sambaqui, Florianópolis, $27^{\circ} 28^{\prime} 36^{\prime \prime}$ S, 48³2'00" W, CCDB 1922; Canto dos Ganchos, Governador Celso Ramos, CCDB 1923; Florianópolis, 2740'51.1" S, 48³3'42.6” W, CCDB 1924; Rio da Cachoeira, Governador Celso Ramos, 27²5’08.3” S, 48³5'47.4" W, CCDB 1929; Ribeirão da Ilha, Florianópolis, 27²'11" S, 48³3'00" W, CCDB 1931. Previous records: Bond-Buckup and Buckup 1989; Ramos-Porto and Coelho 1998; Bond-Buckup and Buckup 1999; Melo 2003; Nazari et al. 2003.

\section{Macrobrachium potiuna (Müller, 1880)}

Specimens in a collection: São Francisco do Sul, UFRGS 0694; Rio Perabeiraba, Joinville, 2607'20.4" S, 4856'22.6" W, UFRGS 2649; Garuva, UFRGS 2659; tributary of Rio Itajaí-Açú, UFRGS 2819; Sanga do Meio, Santa Terezinha, Forquilhinha, UFRGS 2960; tributary of Rio Ratones, Florianópolis, $27^{\circ} 30^{\prime} 40^{\prime \prime}$ S, 48²9'11.25” W, UFRGS 3182; tributary of Rio Lagoa Ribeirão, Paulo Lopes, 2759’33" S, 48 40'38" W, UFRGS 3183; tributary of Rio d'Una, Morro 


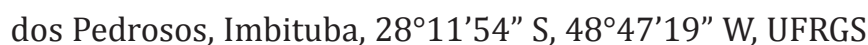
3200; Florianópolis, $27^{\circ} 43^{\prime} 30^{\prime \prime}$ S, 48 $32^{\prime} 18^{\prime \prime}$ W, CCDB 1859; Itajaí, 2657'38.8” S, 48³9'14.4” W, CCDB 1920; Rio Espinheirinho, Itajaí, 2654'33.7" S, 4846'40.4" W, 1921; BR 376, Km 3, Garuva, 2600'17.6” S, 4852'17.1” W, CCDB 2281. Previous records: Müller 1880a; Bond-Buckup and Buckup 1989; Ramos-Porto and Coelho 1998; BondBuckup and Buckup 1999; Ammar et al. 2002; Boos and Althoff 2002; Boos and Althoff 2003; Melo 2003; Nazari et al. 2003.

Palaemon northropi (Rankin, 1898)

Specimens in a collection: Porto Belo, UFRGS 0417, 0418.

Previous records: Ramos-Porto and Coelho 1998.

Palaemon pandaliformes (Stimpson, 1871)

Specimens in a collection: Baía da Babitonga, CEPSUL 327. Previous records: Bond-Buckup and Buckup 1989; Müller et al. 1996; Ramos-Porto and Coelho 1998; Bond-Buckup and Buckup 1999; Melo 2003.

Palaemonetes argentinus Nobili, 1901

Previous records: Bond-Buckup and Buckup 1989; Müller and Araujo 1994; Ramos-Porto and Coelho 1998, BondBuckup and Buckup 1999; Melo 2003.

Periclimenes longicaudatus (Stimpson, 1860)

Specimens in a collection: Enseada de Balneário Camboriú, 2659'07"S, 48³5'58”W, FURG 3256. Previous record: Machado et al. 2010.

Periclimenes paivai Chace, 1969

Previous record: Ramos-Porto and Coelho 1998.

\section{Palicidae Bouvier, 1898}

Palicus alternatus Rathbun, 1897

Previous record: Melo 1996; 2010.

Palicus dentatus (A. Milne-Edwards, 1880)

Previous record: Melo 1996; 2010.

Palicus obesus (A. Milne-Edwards, 1880)

Previous record: Melo 1996; 2010.

Palicus sica (A. Milne-Edwards, 1880)

Previous record: Melo 1996; 2010.

Palinuridae Latreille, 1802

Panulirus laevicauda (Latreille, 1817)

Specimens in a collection: Marine biological reserve Arvoredo, CEPSUL 90; Praia do Côco, Balneário Camboriú, CEPSUL 201.

\section{Pandalidae Haworth, 1825}

\section{Plesionika edwardsii (Brandt, 1851)}

Specimens in a collection: $26^{\circ} 44.11^{\prime} \mathrm{S}, 47^{\circ} 32.27^{\prime} \mathrm{W}, 100 \mathrm{~m}$

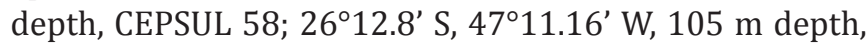
CEPSUL $224 ; 2^{\circ} 05.42^{\prime} \mathrm{S}, 4^{\circ} 56.9^{\prime} \mathrm{W}, 232 \mathrm{~m}$ depth, CEPSUL 227; 2904.68' S, 48³3.09' W, 108 m depth, CEPSUL 228; $26^{\circ} 48.8^{\prime}$ S, 4644,62' W, 208 m depth, CEPSUL 315.

\section{Panopeidae Ortmann, 1893}

Acantholobulus bermudensis (Benedict and Rathbun, 1891)

Previous records: Melo 1996 (cited as Panopeus bermudensis); Karasawa and Schweitzer 2006; Almeida and Coelho 2008.

Acantholobulus schmitti (Rathbun, 1930)

Previous records: Melo 1996; Bouzon and Freire 2007; Almeida and Coelho 2008.

Cyrtoplax spinidentata (Benedict, 1892)

Previous records: Melo 1996; 2010; Almeida and Coelho 2008.

Eurypanopeus abbreviatus (Stimpson, 1860)

Specimens in a collection: Mangue dos Ratones, Florianópolis, UFRGS 1902; Enseada de Itapocorói, Penha, CEPSUL 130. Previous records: Melo 1996; Bouzon and Freire 2007; Almeida and Coelho 2008.

Eurypanopeus dissimilis (Benedict and Rathbun, 1891) Previous record: Melo 1996.

Eurytium limosum (Say, 1818)

Specimens in a collection: Rio Camboriú, Balneário Camboriú, CEPSUL 325. Previous records: Branco 1991; Melo 1996; Almeida and Coelho 2008.

Hexapanopeus angustifrons (Benedict and Rathbun, 1891)

Previous records: Melo 1996; Almeida and Coelho 2008.

Hexapanopeus caribbaeus (Stimpson, 1871)

Previous record: Melo 1996.

Hexapanopeus paulensis Rathbun, 1930

Previous records: Melo 1996; Bouzon and Freire 2007; Almeida and Coelho 2008.

Panopeus americanus Saussure, 1857

Previous records: Melo 1996; Karasawa and Schweitzer 2006; Almeida and Coelho 2008.

Panopeus austrobesus Williams, 1983

Previous records: Melo 1996; Karasawa and Schweitzer 2006.

Panopeus occidentalis Saussure, 1857

Previous records: Melo 1996; Karasawa and Schweitzer 2006; Almeida and Coelho 2008.

Panopeus rugosus A. Milne-Edwards, 1880

Previous records: Melo 1996; Karasawa and Schweitzer 2006; Almeida and Coelho 2008.

Tetraxanthus rathbunae Chace, 1939

Specimens in a collection: $26^{\circ} 18.63^{\prime}$ S, $47^{\circ} 18.12^{\prime}$ W, $100 \mathrm{~m}$ depth, CEPSUL 61. Previous record: Melo 1996; 2010.

\section{Parapaguridae Smith, 1882}

Oncopagurus gracilis (Henderson, 1888)

Previous record: Nucci et al. 2004.

\section{Parastacidae Huxley, 1879}

\section{Parastacus laevigatus Buckup and Rossi, 1980}

Specimens in a collection: Laguna, nearby to Lagoa Imaruí, UFRGS 1137; Cubatão Grande, Joinville, UFRGS 1366; Chácara dos Ipês, Joinville, UFRGS 1369. Previous record: Buckup and Rossi 1980; Buckup 1998; Buckup 2003.

Parastacus saffordi Faxon,1898

Specimens in a collection: Rio Jordão, Siderópolis, UFRGS 271. Previous record: Buckup and Rossi 1980; Buckup 1998; Buckup 2003.

Parastacus varicosus Faxon, 1898

Specimens in a collection: $26^{\circ} 12^{\prime} 30^{\prime \prime}$ S, 48 $40^{\prime} 47^{\prime \prime} \mathrm{W}$, Estrada do Timbé, Joinville, UFRGS 3066, 3067; 2849'19" S, 49²7'18” W, Parque Ecológico Maracajá, Maracajá, 
UFRGS 4786. Previous record: Buckup 2003.

\section{Parthenopidae MacLeay, 1838}

Agolambrus agonus (Stimpson, 1871)

Previous records: Melo 1996 [cited as Parthenope (Parthenope) agona]; Tan and Ng 2007; Almeida and Coelho 2008.

Costalambrus tommasii (Rodrigues da Costa, 1959)

Previous records: Melo 1996 (cited as Heterocrypta tommasii); Bouzon and Freire 2007 (cited as H. tommasii); Tan and Ng 2007.

Heterocrypta lapidea Rathbun, 1901

Previous record: Melo 1996; 2010.

Mesorhea sexspinosa Stimpson, 1871

Previous record: Melo 1996; 2010.

Spinolambrus fraterculus (Stimpson, 1871)

Previous record: Melo 1996 (cited as Parthenope fraterculus); Melo 2010.

Spinolambrus pourtalesii (Stimpson, 1871)

Previous record: Melo 1996 (cited as Parthenope pourtalesii); Melo 2010.

Solenolambrus brasiliensis Rodrigues da Costa, 1961

Previous record: Melo 1996.

\section{Penaeidae Rafinesque, 1815}

\section{Artemesia longinaris Bate, 1888}

Specimens in a collection: $27^{\circ} 13.56^{\prime} \mathrm{S}, 48^{\circ} 25.81^{\prime} \mathrm{W}, 27 \mathrm{~m}$ depth, CEPSUL $205 ; 2^{\circ} 21.31^{\prime} \mathrm{S}, 48^{\circ} 26.73^{\prime} \mathrm{W}, 18 \mathrm{~m}$ depth, CEPSUL 244, 266, 333; $27^{\circ} 10.13^{\prime}$ S, 48²7.89' W, $18 \mathrm{~m}$ depth, CEPSUL 490; Praia Brava, Florianópolis, UFRGS 3315. Previous record: D’Incao 1998; 1999; Branco and Fracasso 2004; Freitas Jr. et al. 2010.

\section{Farfantepenaeus brasiliensis (Latreille, 1817)}

Specimens in a collection: Barra Velha, CEPSUL 143; $27^{\circ} 15.32^{\prime} \mathrm{S}, 48^{\circ} 26.12^{\prime} \mathrm{W}, 21 \mathrm{~m}$ depth, CEPSUL 264; $27^{\circ} 21.31^{\prime} \mathrm{S}, 48^{\circ} 26.73^{\prime} \mathrm{W}, 18 \mathrm{~m}$ depth, CEPSUL 267; $27^{\circ} 06.2^{\prime} \mathrm{S}, 4^{\circ} 05.87^{\prime} \mathrm{W}, 18 \mathrm{~m}$ depth, CEPSUL 269; $27^{\circ} 20.81^{\prime} \mathrm{S}, 46^{\circ} 26.45^{\prime} \mathrm{W}, 19 \mathrm{~m}$ depth, CEPSUL 487; Ponta da Barra, Laguna UFRGS 2327. Previous records: D'Incao 1998; 1999; Tavares 2002b; Branco and Fracasso 2004; Freitas Jr. et al. 2010; Branco et al. 2011.

\section{Farfantepenaeus paulensis (Pérez Farfante, 1967)}

Specimens in a collection: $26^{\circ} 18^{\prime} \mathrm{S}, 48^{\circ} 25^{\prime} \mathrm{W}, 22 \mathrm{~m}$ depth, CEPSUL $149 ; 27^{\circ} 08.08^{\prime} \mathrm{S}, 48^{\circ} 25.83^{\prime} \mathrm{W}, 29 \mathrm{~m}$ depth, CEPSUL 427; $27^{\circ} 20.81^{\prime}$ S, $46^{\circ} 26.45^{\prime}$ W, 19 m depth, CEPSUL 489; Praia da Caieira, Florianópolis, UFRGS 3326; Ponta das Canas, Florianópolis, UFRGS 3346; Florianópolis, UFRGS 3365; Praia Brava, Florianópolis, UFRGS 4020. Previous records: D'Incao 1998; 1999; Branco and Fracasso 2004; Ferreira and Freire 2009; Freitas Jr. et al. 2010; Branco et al. 2011.

\section{Litopenaeus schmitti (Burkenroad, 1936)}

Specimens in a collection: Porto Belo, UFRGS 2323; Lagoa do Mirin, Laguna, UFRGS 2366; Praia Brava, Florianópolis, UFRGS 3313; 2559.67' S, 48³3.69' W, 13 m depth, CEPSUL 11; 26⒒91' S, 48 $28.83^{\prime} \mathrm{W}, 19.5 \mathrm{~m}$ depth, CEPSUL $36 ; 7^{\circ} 15.32^{\prime} \mathrm{S}, 48^{\circ} 26,12^{\prime} \mathrm{W}, 21 \mathrm{~m}$ depth, CEPSUL 263; $27^{\circ} 09.67^{\prime} \mathrm{S}, 4^{\circ} 16.91^{\prime} \mathrm{W}, 49 \mathrm{~m}$ depth, CEPSUL 440; $27^{\circ} 13.98^{\prime} \mathrm{S}, 47^{\circ} 00.35^{\prime} \mathrm{W}, 305 \mathrm{~m}$ depth, CEPSUL 442 ; $27^{\circ} 20.81^{\prime}$ S, $46^{\circ} 26.45^{\prime}$ W, 19 m depth, CEPSUL 488.
Previous records: D’Incao 1998; 1999; Tavares 2002b; Branco and Fracasso 2004; Freitas Jr. et al. 2010; Branco et al. 2011.

Parapenaeus americanus Rathbun, 1901

Specimens in a collection: $26^{\circ} 44.76^{\prime} \mathrm{S}, 4^{\circ} 32.72^{\prime} \mathrm{W}, 99$ m depth, CEPSUL 6; 2638.97' S, 47²9.9' W, 97 m depth, CEPSUL 7; $26^{\circ} 17.45^{\prime} \mathrm{S}, 47^{\circ} 30.08^{\prime} \mathrm{W}, 80 \mathrm{~m}$ depth, CEPSUL 8; $26^{\circ} 18.79^{\prime} \mathrm{S}, 47^{\circ} 18.79^{\prime} \mathrm{W}, 100 \mathrm{~m}$ depth, CEPSUL 9; $25^{\circ} 58.82^{\prime} \mathrm{S}, 48^{\circ} 25.55^{\prime} \mathrm{W}, 19 \mathrm{~m}$ depth, CEPSUL 37. Previous record: D’Incao 1998; 1999.

Penaeopsis serrata Bate, 1881

Previous record: D’Incao 1998; 1999.

Rimapenaeus constrictus (Stimpson, 1871)

Specimens in a collection: $26^{\circ} 11.71^{\prime} \mathrm{S}, 47^{\circ} 47.61^{\prime} \mathrm{W}$,

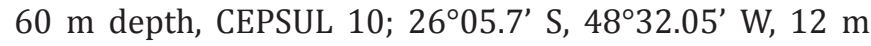
depth, CEPSUL $34 ; 26^{\circ} 11.79^{\prime} \mathrm{S}, 4^{\circ} 47.74^{\prime} \mathrm{W}, 61 \mathrm{~m}$ depth, CEPSUL $39 ; 7^{\circ} 15.32^{\prime} \mathrm{S}, 48^{\circ} 26,12^{\prime} \mathrm{W}, 21 \mathrm{~m}$ depth, CEPSUL 265; $27^{\circ} 20.81^{\prime}$ S, 46 $26,45^{\prime}$ W, 19 m depth, CEPSUL 486. Previous record: D'Incao 1998; 1999; Tavares 2002b; Branco and Fracasso 2004; Freitas Jr. et al. 2010.

Xiphopenaeus kroyeri (Heller, 1862)

Specimens in a collection: $27^{\circ} 10.13^{\prime} \mathrm{S}, 4^{\circ} 27.89^{\prime} \mathrm{W}, 18$ $m$ depth, CEPSUL 492; Porto Belo, UFRGS 2378. Previous record: D'Incao 1998; 1999; Branco et al.1999; Tavares 2002b; Branco and Fracasso 2004; Branco 2005; Freitas Jr. et al. 2010.

\section{Pilumnidae Samouelle, 1819}

Pilumnus caribaeus Desbonne and Schramm, 1867

Previous records: Melo 1996; Karasawa and Schweitzer 2006; Almeida and Coelho 2008.

Pilumnus dasypodus Kingsley, 1879

Previous records: Melo 1996; Branco and Fracasso 2004; Karasawa and Schweitzer 2006; Almeida and Coelho 2008. Pilumnus diomedae Rathbun, 1894

Previous records: Melo 1996; Karasawa and Schweitzer 2006; Bouzon and Freire 2007.

Pilumnus quoii H. Milne Edwards, 1834

Specimens in a collection: Enseada de Itapocorói, Penha, CEPSUL 114.

\section{Pilumnus reticulatus Stimpson, 1860}

Previous records: Melo 1996; Karasawa and Schweitzer 2006; Bouzon and Freire 2007; Almeida and Coelho 2008. Pilumnus spinosissimus Rathbun, 1898

Previous records: Melo 1996; Karasawa and Schweitzer 2006; Bouzon and Freire 2007; Almeida and Coelho 2008.

\section{Pilumnoididae Guinot and Macpherson, 1987}

\section{Pilumnoides bassleri A. Milne-Edwards, 1880}

Previous records: Melo 1996; Karasawa and Schweitzer 2006.

Pilumnoides coelhoi Guinot and Macpherson, 1987

Specimens in a collection: Enseada de Itapocorói, Penha, CEPSUL 128. Previous records: Melo 1996; Karasawa and Schweitzer 2006; Almeida and Coelho 2008.

Pinnotheridae De Haan, 1833

Austinixa patagoniensis (Rathbun, 1918)

Previous record: Melo 1996 (cited as Pinnixa patagoniensis); 
Alves and Pezzuto 2009.

Clypeasterophilus stebbingi (Rathbun, 1918)

Specimens in a collection: Marine biological reserve Arvoredo, FURG 913. Previous record: Martins and D'Incao 1996.

\section{Dissodactylus crinitichelis Moreira, 1901}

Previous record: Melo 1996.

Fabia emiliai (Melo, 1971)

Previous record: Melo 1996.

Holothuriophilus tomentosus (Ortmann, 1894)

Specimens in a collection: Florianópolis, Enseada de Ratones, 2727' S, 48³2' W, MZUSP 15752, 15753. Previous records: Melo and Boehs 2004.

Pinnixa brevipollex Rathbun, 1898.

Specimens in a collection: Santa Catarina, DOUFPe (N.Oc. Alm. Saldanha Est. 2217, cited as Pinnixa rapax). Previous record: Melo 1990 (cited as P. rapax); Martins and D'Incao 1996; Melo 1996 (cited as P. rapax).

Pinnixa chaetopterana Stimpson, 1860

Previous record: Melo 1996.

Pinnixa sayana Stimpson, 1860

Previous record: Martins and D'Incao 1996; Melo 1996.

Tumidotheres maculatus (Say, 1818)

Specimens in a collection: Praia da Daniela, Florianópolis, MZUSP 9552. Previous record: Martins and D'Incao 1996; Bouzon and Freire 2007.

Zaops ostreus (Say, 1817)

Previous record: Melo 1996 (cited as Z. ostreum).

\section{Pisidae Dana, 1851}

\section{Libinia ferreirae Brito Capello, 1871}

Specimens in a collection: $25^{\circ} 56.82^{\prime} \mathrm{S}, 48^{\circ} 34.25^{\prime} \mathrm{W}, 10 \mathrm{~m}$ depth, CEPSUL 43; 2614'34.76" S, 48³8'25.98” W, CCDB 3055. Previous record: Melo 1996; 2010; Branco and Fracasso 2004.

\section{Libinia spinosa $\mathrm{H}$. Milne Edwards, 1834}

Specimens in a collection: $29^{\circ} 04.45^{\prime} \mathrm{S}, 4^{\circ} 29.21^{\prime} \mathrm{W}, 18$ $\mathrm{m}$ depth, CEPSUL 189; $27^{\circ} 08.36^{\prime} \mathrm{S}, 48^{\circ} 26.24^{\prime} \mathrm{W}, 28 \mathrm{~m}$ depth, CEPSUL $345 ; 7^{\circ} 11.47^{\prime} \mathrm{S}, 48^{\circ} 20.78^{\prime} \mathrm{W}, 39$ m depth, CEPSUL $346 ; 2^{\circ} 11.57^{\prime} \mathrm{S}, 48^{\circ} 20.67^{\prime} \mathrm{W}$, $38 \mathrm{~m}$ depth, CEPSUL 466; $27^{\circ} 16.55^{\prime}$ S, $48^{\circ} 19.33^{\prime}$ W, 41 m depth, CEPSUL 467; Ponta das Canas, Florianópolis, UFRGS 3070; Praia Brava, Florianópolis, UFRGS 3436; Santa Catarina (Projeto "RPC") MZUSP 4338. Previous records: Melo 1990; 1996; 2010; Branco and Fracasso 2004; Freitas Jr. et al. 2010.

Nibilia antilocapra (Stimpson, 1871)

Previous record: Melo 1996.

Pelia rotunda A. Milne-Edwards, 1875

Previous records: Melo 1996; 2010; Bouzon and Freire 2007.

Rochinia crassa (A. Milne-Edwards, 1879)

Specimens in a collection: $28^{\circ} 40.33^{\prime} \mathrm{S}, 4^{\circ} 19.87^{\prime} \mathrm{W}, 346$ $m$ depth, CEPSUL 179, 181; $27^{\circ} 36.38^{\prime} \mathrm{S}, 4^{\circ} 45.37^{\prime} \mathrm{W}, 468$ $\mathrm{m}$ depth, CEPSUL 180; $26^{\circ} 50.82^{\prime}$ S, 46²8.19' W, 390 m depth, CEPSUL 182.

Rochinia gracilipes A. Milne-Edwards, 1875

Previous record: Melo 1996; 2010.

\section{Platyxanthidae Guinot, 1977}

Platyxanthus crenulatus A. Milne-Edwards, 1879
Specimens in a collection: "Armação", MZUSP 6459. Previous records: Melo 1990; 1996; Karasawa and Schweitzer 2006.

\section{Porcellanidae Haworth, 1825}

Megalobrachium roseum (Rathbun, 1900)

Specimens in a collection: Praia do Sambaqui, Florianópolis, 29²9'12.1" S, 48³2'20.0” W, CCDB 1932.

Minyocerus angustus (Dana, 1852)

Previous records: Melo 1999; Bouzon and Freire 2007.

Pachycheles chubutensis Boschi, 1963

Previous record: Melo 1999.

Pachycheles laevidactylus Ortmann, 1892

Specimens in a collection: Ponta das canas, Florianópolis, UFRGS 1391; Santa Marta Grande Cape, CEPSUL 129; Praia de Cabeçudas, Itajaí, 2655'46.2” S, 48³7'48.9” W, CCDB 1933; Praia da Conceição, Bombinhas, 27²12'10.3" S, 48²9'22.0" W, CCDB 1934; Praia do Sambaqui, Florianópolis, 27²9'12.7" S, 48³2'20.0” W, CCDB 1935; Praia Brava, Itajaí, 2657'34.0” S, 48³7’43.4” W, CCDB 1936; Bombinhas, CCDB 3124; Ilha de São Francisco, MZUSP 9984; Praia Grande, São Francisco do Sul, MZUSP 9020. Previous records: Veloso and Melo 1993 (cited as Pachycheles haigae); Melo 1999; Veloso 1999.

Pachycheles monilifer (Dana, 1852)

Previous record: Melo 1999.

Petrolisthes armatus (Gibbes, 1850)

Specimens in a collection: Enseada de Itapocorói, Penha, CEPSUL 127; Praia da Conceição, Bombinhas, 27²12'10.3" S, 48²9'22.0" W, CCDB 1882; Praia de Cabeçudas, Itajaí, $26^{\circ} 55^{\prime} 46.2^{\prime \prime}$ S, 48³7'48.9” W, CCDB 1883; Praia do Sambaqui, Florianópolis, 27²9'12.7” S, 48³2'20.0" W, CCDB 1894, 2979; Praia dos Ingleses, Bombinhas, 2708'39.7" S, 48²8'38.2” W, CCDB 1895. Previous record: Melo 1999; Mantelatto et al. 2011.

Petrolisthes galathinus (Bosc, 1802)

Previous record: Melo 1999.

Polyonyx gibbesi Haig, 1956

Previous records: Melo 1999; Veloso 1999.

Porcellana sayana (Leach, 1820)

Specimens in a collection: $26^{\circ} 31.74^{\prime} \mathrm{S}, 48^{\circ} 19.9^{\prime} \mathrm{W}, 40 \mathrm{~m}$ depth, CEPSUL 19; $26^{\circ} 05.66^{\prime} \mathrm{S}, 48^{\circ} 06.4^{\prime} \mathrm{W}, 40 \mathrm{~m}$ depth, CEPSUL $20 ; 2^{\circ} 59.12^{\prime} \mathrm{S}, 48^{\circ} 25.72^{\prime} \mathrm{W}, 20 \mathrm{~m}$ depth, CEPSUL 21; $26^{\circ} 17.31^{\prime} \mathrm{S}, 48^{\circ} 10.79^{\prime} \mathrm{W}, 42 \mathrm{~m}$ depth, CEPSUL 51; $26^{\circ} 31.74^{\prime} \mathrm{S}, 48^{\circ} 19.9^{\prime} \mathrm{W}, 40 \mathrm{~m}$ depth, CEPSUL 317. Previous records: Melo 1999; Veloso 1999; Branco and Fracasso 2004.

\section{Portunidae Rafinesque, 1815}

\section{Achelous ordwayi (Stimpson, 1860)}

Previous record: Melo 1996 (cited as Portunus ordwayi).

Achelous spinicarpus (Stimpson, 1871)

Previous record: Melo 1996; 2010 (cited as Portunus spnicarpus).

Achelous spinimanus (Latreille, 1819)

Specimens in a collection: Baía da Babitonga, CEPSUL 116; $26^{\circ} 25.68^{\prime} \mathrm{S}, 48^{\circ} 22.95^{\prime} \mathrm{W}, 31-35 \mathrm{~m}$ depth, CEPSUL $123 ; 27^{\circ} 21.31^{\prime} \mathrm{S}, 48^{\circ} 26.72^{\prime} \mathrm{W}, 90 \mathrm{~m}$ depth, CEPSUL 314; $27^{\circ} 16.73^{\prime} \mathrm{S}, 48^{\circ} 19.11^{\prime} \mathrm{W}, 42 \mathrm{~m}$ depth, CEPSUL 348; $27^{\circ} 08.78^{\prime}$ S, $48^{\circ} 24.74^{\prime}$ W, 32 m depth, CEPSUL 465; 
Garopaba, UFRGS 1924; Praia dos Ingleses, Florianópolis, UFRGS 1925; Ponta das Canas, Florianópolis, UFRGS 3484. Previous records: Melo 1996; 2010; Branco and Fracasso 2004; Freitas Jr. et al. 2010 (cited as Portunus spinimanus). Arenaeus cribrarius (Lamarck, 1818)

Specimens in a collection: $27^{\circ} 21.31^{\prime} \mathrm{S}, 48^{\circ} 26.72^{\prime} \mathrm{W}, 70$ $m$ depth, CEPSUL 313; $26^{\circ} 11.046^{\prime} \mathrm{S}, 48^{\circ} 28.005^{\prime} \mathrm{W}$, CCDB 3516. Previous records: Melo 1996; 2010; Tavares 2002c; Branco and Fracasso 2004.

Callinectes bocourti A. Milne-Edwards, 1879

Specimens in a collection: $26^{\circ} 27.72^{\prime} \mathrm{S}, 48^{\circ} 31.03^{\prime} \mathrm{W}, 20$ $m$ depth, CEPSUL 12 ; 28 $27^{\circ} 49.57^{\prime \prime} \mathrm{S}, 48^{\circ} 47^{\prime} 57.75^{\prime \prime} \mathrm{W}$, UFRGS 4760; 2856'57.18” S, 4849'7.70” W, UFRGS 4762. Previous records: Branco 1991; Melo 1996; Tavares 2002c; Freitas Jr. et al. 2010; Branco et al. 2011.

\section{Callinectes danae (Smith, 1869)}

Specimens in a collection: shelf adjacent to Baía da Babitonga, 75 m depth, CEPSUL 307; Rio Camboriú, Balneário Camboriú, CEPSUL 322; 27016.32' S, 48²6.12' W, $21 \mathrm{~m}$ depth, CEPSUL 357; 26⒓24' S, 48³6.36' W, 20-100 m depth, CEPSUL 401; Baía da Babitonga, CEPSUL 402; $26^{\circ} 16.35^{\prime}$ S, $48^{\circ} 40.54^{\prime} \mathrm{W}, 20-100 \mathrm{~m}$ depth, CEPSUL 405; Laguna, UFRGS 1938; 28²7'49.57' S, 4847'57.75" W, UFRGS 4757; 28²7'51.57"S, 4850'25.18” W, UFRGS 4758; 28 $56^{\prime} 57.18^{\prime \prime}$ S, 4849'7.70" W, UFRGS 4759. Previous records: Branco 1991; Branco and Masunari 1992; Melo 1996; 2010; Tavares 2002c; Branco and Fracasso 2004; Freitas Jr. et al. 2010; Branco et al. 2011.

\section{Callinectes exasperatus (Gerstaecker, 1856)}

Previous records: Branco 1991; Melo 1996; Tavares 2002c. Callinectes ornatus (Ordway, 1863)

Specimens in a collection: $26^{\circ} 25.68^{\prime} \mathrm{S}, 48^{\circ} 22.95^{\prime} \mathrm{W}, 31-35$ $m$ depth, CEPSUL 111; shelf adjacent to Baía da Babitonga, $75 \mathrm{~m}$ depth, CEPSUL 306; 26⒓24' S, 48³6.36' W,20$100 \mathrm{~m}$ depth, CEPSUL 403; Baía da Babitonga, CEPSUL 404; 2708.79' S, 48²4.74' W, 32 m depth, CEPSUL 464. Previous records: Branco 1991; Melo 1996; 2010; Tavares 2002c; Branco and Fracasso 2004; Freitas Jr. et al. 2010; Branco et al. 2011.

\section{Callinectes sapidus Rathbun, 1896}

Specimens in a collection: $27^{\circ} 16.61^{\prime} \mathrm{S}, 48^{\circ} 18.00^{\prime} \mathrm{W}, 47$ $\mathrm{m}$ depth, CEPSUL 332; estuary of Rio Itajaí-Açú, CEPSUL 506; Laguna, CCDB 994; Praia da Mutuca, Florianópolis, 2741'49.4"S, 48³3'11.0”W, CCDB 1890. Previous records: Branco 1991; Melo 1996; 2010; Tavares 2002c; Branco and Fracasso 2004; Freitas Jr. et al. 2010; Branco et al. 2011.

\section{Charybdis hellerii (A. Milne-Edwards, 1867)}

Specimens in a collection: $26^{\circ} 57.45^{\prime} \mathrm{S}, 4^{\circ} 37.31^{\prime} \mathrm{W}$, CEPSUL 101; 26²6.53' S, 48 ${ }^{\circ} 36.13^{\prime} \mathrm{W}$, CEPSUL102, 103; $26^{\circ} 49.67^{\prime} \mathrm{S}, 48^{\circ} 36.75^{\prime} \mathrm{W}$, CEPSUL 104; 2651.1' S, 48³5,72' W, CEPSUL 105; Baía da Babitonga CEPSUL 108, 121; Praia da Pinheira, Palhoça, CEPSUL 323; Enseada de Itapocorói, Penha, CEPSUL 377, 378, 379, 380, 381, 382, 383, 384; Praia do Sambaqui, Florianópolis, 27²9'12.7" S, 48³2'20.0" W, CCDB 1888; Caieira da Barra, Florianópolis, 273' 48.85”S, 48³2'58.03" W, CCDB 2909. Previous records: Mantelatto and Dias 1999; Boos et al. 2010; Freitas Jr. et al. 2010. Note: Exotic species.

Coenophthalmus tridentatus A. Milne-Edwards, 1879

Previous record: Melo 1996.

Cronius ruber (Lamarck, 1818)
Previous records: Melo 1996; 2010; Branco and Fracasso 2004; Bouzon and Freire 2007.

Ovalipes trimaculatus (De Haan, 1833)

Specimens in a collection: CEPSUL 109, 110. Previous record: Melo 1996; 2010.

\section{Processidae Ortmann, 1890}

Ambidexter symmetricus Manning and Chace, 1971

Previous record: Christoffersen 1998.

Processa hemphilli Manning and Chace, 1971

Previous records: Christoffersen 1998; Bond-Buckup and Buckup 1999.

\section{Raninidae De Haan, 1839}

Ranilia constricta (A. Milne-Edwards, 1880)

Previous record: Bond-Buckup and Buckup 1999.

\section{Scyllaridae Latreille, 1825}

\section{Scyllarides brasiliensis Rathbun, 1906}

Specimens in a collection: Baía do Farol, Marine biological reserve Arvoredo, MZUSP 16260. Previous record: Dall'Occo et al. 2007; Santana et al. 2007; Tavares et al. 2009.

\section{Scyllarides deceptor Holthuis, 1963}

Specimens in a collection: $29^{\circ} 03.84^{\prime}$ S, $49^{\circ} 01.94^{\prime}$ W, $54 \mathrm{~m}$ depth, CEPSUL 203. Previous records: Melo 1996; 1999; Oliveira et al. 2008.

\section{Sergestidae Dana, 1852}

\section{Acetes americanus Ortmann, 1893}

Previous records: D'Incao 1998; 1999; Branco and Fracasso 2004.

Peisos petrunkevitchi (Burkenroad, 1945)

Previous records: D’Incao 1998; 1999; Melo 1999.

\section{Sesarmidae Dana, 1851}

Aratus pisonii (H. Milne-Edwards, 1837)

Specimens in a collection: $28^{\circ} 25^{\prime} 47.47^{\prime \prime} \mathrm{S}, 48^{\circ} 50^{\prime} 43.54^{\prime \prime}$ W, UFRGS 4766; 28³0'13.94” S, 4846'02.99” W, UFRGS 4768. Previous records: Branco 1991.

Armases angustipes (Dana, 1852)

Previous records: Melo 1996.

Armases rubripes (Rathbum, 1897)

Specimens in a collection: Praia Brava, Florianópolis, UFRGS 2296, 3433. Previous records: Branco 1991 (cited as Metasesarma rubripes); Melo 1996 (cited as M. rubripes); Almeida and Coelho 2008.

Sesarma rectum Randall, 1840

Previous records: Melo 1996; Branco et al. 2011.

\section{Sicyoniidae Ortmann, 1898}

\section{Sicyonia dorsalis (Bate, 1878)}

Specimens in a collection: $25^{\circ} 55.41^{\prime} \mathrm{S}, 48^{\circ} 33.57^{\prime} \mathrm{W}, 11 \mathrm{~m}$ depth, CEPSUL 29; $26^{\circ} 25.92^{\prime} \mathrm{S}, 48^{\circ} 34.76^{\prime} \mathrm{W}, 12 \mathrm{~m}$ depth, CEPSUL 53; $27^{\circ} 13.56^{\prime} \mathrm{S}, 48^{\circ} 25.81^{\prime} \mathrm{W}, 27 \mathrm{~m}$ depth, CEPSUL 204; $27^{\circ} 08.62^{\prime} \mathrm{S}, 48^{\circ} 26.36^{\prime} \mathrm{W}, 21 \mathrm{~m}$ depth, CEPSUL 
295; $27^{\circ} 08.08^{\prime} \mathrm{S}, 48^{\circ} 25.83^{\prime} \mathrm{W}, 29 \mathrm{~m}$ depth, CEPSUL 426; $27^{\circ} 08.15^{\prime}$ S, $48^{\circ} 25.96^{\prime}$ W, 27 m depth, CEPSUL 493. Previous record: D'Incao 1998; Branco and Fracasso 2004; Freitas Jr. et al. 2010.

\section{Sicyonia laevigata Stimpson, 1871}

Previous record: D’Incao 1998.

Sicyonia typica (Boeck, 1864)

Specimens in a collection: $26^{\circ} 18.48^{\prime}$ S, $48^{\circ} 11.51^{\prime} \mathrm{W}, 41 \mathrm{~m}$ depth, CEPSUL 48; Florianópolis, UFRGS 4558. Previous records: D’Incao 1998; Tavares 2002b.

\section{Solenoceridae Wood-Mason, 1891}

\section{Pleoticus muelleri (Bate, 1888)}

Specimens in a collection: $26^{\circ} 41,00^{\prime} \mathrm{S}, 4^{\circ} 43.53^{\prime} \mathrm{W}, 81$ $\mathrm{m}$ depth, CEPSUL $31 ; 26^{\circ} 29.44^{\prime} \mathrm{S}, 47^{\circ} 37.66^{\prime} \mathrm{W}, 80 \mathrm{~m}$ depth, CEPSUL 38; $26^{\circ} 24.64^{\prime} \mathrm{S}, 47^{\circ} 52.5^{\prime} \mathrm{W}, 60 \mathrm{~m}$ depth, CEPSUL $40 ; 7^{\circ} 15.32^{\prime} \mathrm{S}, 48^{\circ} 26.12^{\prime} \mathrm{W}, 21 \mathrm{~m}$ depth, CEPSUL 262; $27^{\circ} 21.31^{\prime} \mathrm{S}, 48^{\circ} 26.73^{\prime} \mathrm{W}, 18 \mathrm{~m}$ depth, CEPSUL 268; $27^{\circ} 08.62^{\prime} \mathrm{S}, 48^{\circ} 26.36^{\prime} \mathrm{W}, 21 \mathrm{~m}$ depth, CEPSUL 296; 27¹0.13' S, 48²7.89' W, 18 m depth, CEPSUL 491. Previous record: D'Incao 1998; 1999; Branco and Fracasso 2004; Freitas Jr. et al. 2010; Branco et al. 2011.

Solenocera necopina Burkenroad, 1939

Previous record: D'Incao 1998.

\section{Trichodactylidae H. Milne Edwards, 1853}

\section{Trichodactylus dentatus H. Milne Edwards, 1853}

Specimens in a collection: Rio Humboldt, Joinville, NHM uncatalogued; tributary of Rio Itapocuzinho, Guaramirim, MNRJ MD-1261; Joinville, 2612'30” S, 4850’47” W, INPA 875; roadway to Timbé, Joinville, UFRGS 3101; Chaparral, Joinville, UFRGS 3261. Previous record: Magalhães 2003.

\section{Trichodactylus fluviatilis Latreille, 1828}

Specimens in a collection: Rio Espingarda, National Park Serra do Itajaí, Indaial, UFRGS 3564, 3565, 3566, 3567, 3568, 3569, 3572; Rio "Vananal" (probably Bananal) USNM 71265; Rio Cubatão, Águas Mornas, NHMW 6646; Águas Mornas, MNHN BP-145; Águas Mornas, ZMB 15798; Águas Mornas, ZMB 15797; Novo Horizonte, Lauro Müller, MZUSP 6333; Novo Horizonte, Lauro Müller, INPA 563; São Francisco do Sul, USNM 76758; Joinville, MZUSP 274; Rio Humboldt, Corupá, ZMH K-3568a-b; Rio Humboldt, Corupá, ZMH K-5225; Rio Humboldt, Corupá, BMNH 1910.7.19.1-5; Rio Humboldt, Corupá, NHM 1910.7.29.720; Rio Humboldt, Corupá, NHM 1910.7.29.6-11; Rio Humboldt, Corupá, BMNH 1923.7.26.1-15; Águas Mornas, MNHN BP-145; Águas Mornas, ZMB 15798; Águas Mornas, ZMB 15797; Novo Horizonte, Lauro Müller, MZUSP 6333; Novo Horizonte, Lauro Müller, INPA 563; Rio Troncos, tributary of Rio da Prata, Joinville, UFRGS 1053; Joinville, SMF 4321; Jaraguá do Sul, ZMH K-3622; Jaraguá do Sul, ZMH K-3566; Rio Itapocú basin, ZMH K-3565; Jaraguá do Sul, MCSNM 1006; Blumenau, ZMB 8530; Blumenau ZMB 8411; Novo Horizonte, Lauro Müller, MZUSP 6333; Novo Horizonte, Lauro Müller, INPA 563; Alto Palmeiras, Rio dos Cedros MZUSP 6375; Alto Palmeiras, Rio dos Cedros, MZUSP 9508; Timbé do Sul, MZUSP 6294; Praia Grande, Faxinal, UFRGS 1065; roadway to Timbé, Joinville, $26^{\circ} 12^{\prime} 30^{\prime \prime} \mathrm{S}, 48^{\circ} 50^{\prime} 47^{\prime \prime} \mathrm{W}$, INPA 874; roadway to Timbé, Joinville, $26^{\circ} 12^{\prime} 30^{\prime \prime}$ S, 4850’47” W, UFRGS 3064, 3068;
Mountain of the Juvenato, Jaraguá do Sul, UFRGS 2380; Rio Araranguá basin, Nova Veneza, 2840'41"S, 49³6'24"W, UFRGS 3428; Santa Catarina, MNRJ MD-1272; Santa Catarina, NHMW 6647. Previous record: Moreira 1903, Melo 1967, Bott 1969.

\section{Trichodactylus kensleyi Rodríguez, 1982}

Specimens in a collection: Rio Iguaçú, Porto União, MZUSP 2302; Arroio Santa Fé, tributary of Rio Uruguai, Itapiranga, UFRGS 1075. Previous record: Magalhães 2003.

Trichodactylus panoplus (von Martens, 1869)

Specimens in a collection: Porto Belo, MCT-PUCRS 788. Previous record: Magalhães 2003.

\section{Trichodactylus petropolitanus (Göldi, 1886)}

Specimens in a collection: Rio Pirabeiraba, Joinville, UFRGS 851; Rio das Onças, Garuva, MHNCI 590, 591; Joinville, MHNCI 21, Joinville, MHNG, Joinville, SMF 4326, Joinville, SMF 4324, Joinville, DZ-MZUSP 10743; Rio da Prata, Joinville, UFRGS 1063; "Bananal", MNRJ MD-1174; Rio Novo, tributary of Rio Itapocu, SMF 4325; Rio Novo, tributary of Rio Itapocu, ZMH K-3572; Rio Itapocu basin, Jaraguá do Sul, ZMH K-3618; tributary of do Rio Itapocu, ZMH K-3571; Rio Humboldt basin, nearly Joinville, ZMH K-6770; Rio Humboldt basin, nearly Joinville, ZMH K-6727; Rio Humboldt basin, nearly Joinville, ZMH K-3568a; Corupá, SMF 4323; Corupá, ZMB 21541; Joinville, UFRGS 1054; Rio Humboldt, BMNH 1923.7.26.16-18; Rio Troncos, Joinville, UFRGS 1055; Rio Fleith, tributary of Rio Cubatão, Joinville, UFRGS 1056; Rio Tronn, Joinville, UFRGS 1058; Rio Tronn, Joinville, UFRGS 1059; Rio Tronn, Joinville, UFRGS 1060; Águas Mornas, NHMW 6650; Rio Cubatão, Joinville, 2313'23" S, 4850'47" W, INPA 876; Rio Cubatão,

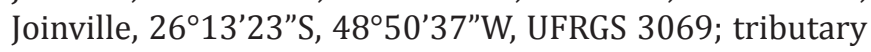

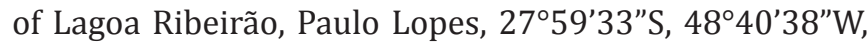

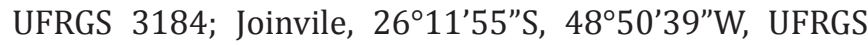
3554; Santa Catarina, ZMH K-3698, Santa Catarina, MZUSP 1788; Santa Catarina, MZUSP 319. Previous record Bott 1969.

\section{Trichopeltariidae Tavares and Cleva, 2010}

\section{Trichopeltarion pezzutoi Tavares and Melo, 2005}

Specimens in a collection: Santa Marta Grande Cape, 400$500 \mathrm{~m}$ depth (type locality), MZUSP 16714. Previous record: Tavares and Melo 2005; Tavares and Cleva 2010.

\section{Upogebiidae Borradaile, 1903}

Upogebia brasiliensis Holthuis, 1956

Previous record: Melo 1999.

Upogebia omissa Gomes Corrêa, 1968

Previous record: Melo 1999.

Varunidae H. Milne Edwards, 1853

Cyclograpsus integer H. Milne Edwards, 1837

Previous records: Almeida and Coelho 2008; Melo 1996.

Xanthidae MacLeay, 1838

Allactaea lithostrota Williams, 1974

Previous record: Melo 1996.

Cataleptodius floridanus (Gibbes, 1850) 
Previous records: Melo 1996; Bouzon and Freire 2007; Almeida and Coelho 2008.

Edwardsium spinimanus (H. Milne Edwards, 1834)

Previous records: Melo 1996; Almeida and Coelho 2008.

Speocarcinus amazonicus Brandão, Tavares and Coelho Filho, 2010

Specimens in a collection: Porto Belo, MZUSP 22789.

Previous record: Brandão et al. 2010.

Speocarcinus carolinensis Stimpson, 1859

Specimens in a collection: Enseada de Balneário Camboriú, 2659'07" S, 48³5'58” W, FURG 3255. Previous record: Machado et al. 2009.

\section{Isopoda Latreille, 1817}

\section{Armadillidae Brandt, 1831}

\section{Cubaris murina Brandt, 1833}

Specimens in a collection: Timbó, 26²9'39.82" S, 4916'13.56" W, UFRGS 1499; Blumenau, 2655'02.28” S, 49 04'28.14" W, UFRGS 1500. Previous record: Araujo et al. 1996; Souza-Kury 1998.

\section{Armadillidiidae Brandt, 1833}

\section{Armadillidium vulgare (Latreille, 1804)}

Specimens in a collection: São Lourenço do Oeste, 2621'15.01" S, 5250'58.31" W, UFRGS 1582; Anchieta, $26^{\circ} 32^{\prime} 07.61^{\prime \prime}$ S, 5319'48.45” W, UFRGS 1771, São Miguel d'Oeste, 2643'52.85" S, 5331'08.57" W, UFRGS 1591;

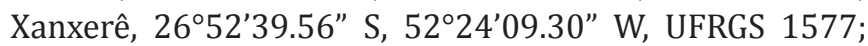
Faxinal dos Guedes, $26^{\circ} 51^{\prime} 09.71^{\prime \prime}$ S,52 $2^{\circ} 15^{\prime} 36.32^{\prime \prime}$ W, UFRGS

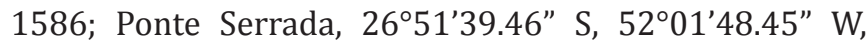
UFRGS1583; Blumenau, 2655'02.28” S, 4904'28.14” W, UFRGS 1588; Santa Cecília, 2657'54.03" S, 50²5'11.39"

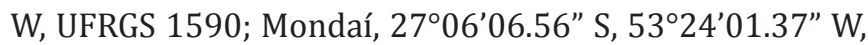
UFRGS 1575; Catanduvas, $27^{\circ} 04^{\prime} 19.71^{\prime \prime} \mathrm{S}, 5^{\circ} 39^{\prime} 31.47^{\prime \prime}$

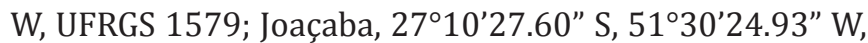
UFRGS 1580; Itá, $27^{\circ} 16^{\prime} 36.19^{\prime \prime}$ S, 52¹9'55.24" W, UFRGS

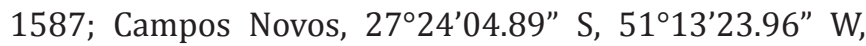
UFRGS 1581; Florianópolis, 27³5'47.88” S, 48³1'59.23"

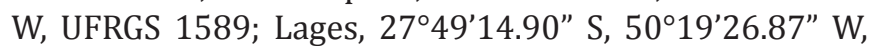
UFRGS 1585; Bom Retiro, 2747'57.51" S, 49²9'22.04" W, UFRGS 1587; Urussanga, 28³1'18.85" S, 49¹9'14.83" W, UFRGS 1584; Sombrio, 2906'22.63" S, 49³8'07.22” W, UFRGS 1576. Previous record: Araujo et al. 1996; SouzaKury 1998; Araujo 1999. Note: Exotic species.

\section{Balloniscidae Vandel, 1963}

\section{Balloniscus sellowii (Brand, 1833)}

Specimens in a collection: Papanduva, 26 24'25.71" S, 5008'32.52" W, UFRGS 1787; Campo Erê, 26²3'44.59" S, 5304'54.29" W, UFRGS 1780; São Lorenço d'Oeste, 2621'15.01" S, 52 50 '58.31" W, UFRGS 1779; Anchieta, $26^{\circ} 32^{\prime} 07.61^{\prime \prime}$ S, 5319'48.45" W, UFRGS 1781; São Miguel d'Oeste, 2643'52.85" S, 5331'08.57" W, UFRGS 1782;

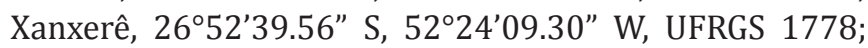
Faxinal dos Guedes, 26 $51^{\prime} 09.71^{\prime \prime}$ S, 52 $2^{\circ} 15^{\prime} 36.32^{\prime \prime} \mathrm{W}$, UFRGS 1777; Ponte Serrada, 2651'39.46" S, 5201'48.45" W, UFRGS1776; Irani, $27^{\circ} 01^{\prime} 15.38^{\prime \prime}$ S, 51 $53^{\prime} 04.68^{\prime \prime}$ W,
UFRGS 1775; Santa Cecília, 2657'54.03” S, 50²5'11.39” W, UFRGS 1795; Chapecó, 2705' 48.43” S, 52³7’06.42” W,

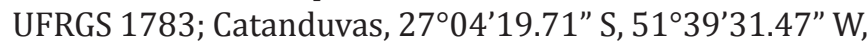
UFRGS 1774; Itá, $27^{\circ} 16^{\prime} 36.19^{\prime \prime}$ S, 52¹9'55.24" W, UFRGS

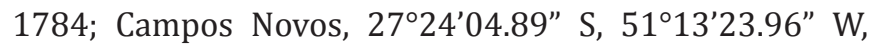
UFRGS 1773; Florianópolis, 27³5'47.88” S, 48³1'59.23" W, UFRGS 1786; Lages, 2749'14.90” S, 50¹9'26.87” W, UFRGS 1772; Bom Retiro, 2747'57.51" S, 49²9'22.04"

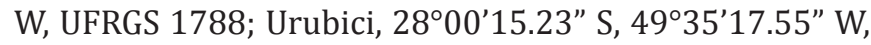
UFRGS 1789; São Joaquim, 28¹7'32.20” S, 4956’05.48” W, UFRGS 1794; Bom Jardim da Serra, 28²0'19.97" S, 49³7'30.87" W, UFRGS 1790; Laguna, 28²9’01.89” S, 4846'50.74" W, UFRGS 1793; Sombrio, 2906'22.63" S, 49³8'07.22" W, UFRGS 1786; Praia Grande, 29¹1'42.68" S, 4957'09.45" W, UFRGS 1791. Previous record: Araujo et al. 1996; Souza-Kury 1998; Araujo 1999.

\section{Bathytropidae Vandel, 1952}

Neotroponiscus argentinus (Giambiagi de Calabrese, 1939)

Specimens in a collection: Nova Teutônia, Itá, 27²16'36.19” S, 52¹9'55.24" W, SMNH. Previous record: Andersson 1960; Souza-Kury 1998.

Neotroponiscus plaumanni (Andersson, 1960)

Specimens in a collection: Nova Teutônia, Itá, 27 $16^{\prime} 36.19^{\prime \prime}$ S, 52 $19^{\prime} 55.24 "$ W, SMNH. Previous record: Andersson 1960; Souza-Kury 1998.

\section{Bopyridae Rafinesque, 1815}

\section{Anathelges resupinatus (Müller, 1871)}

Previous record: Brasil-Lima 1998 (parasite of Pagurus sp.)

\section{Bopyrina abbreviata Richardson, 1904}

Previous record: Markham 1988; Brasil-Lima 1998 (infesting H. obliquimanus).

Probopyria alphei (Richardson, 1900)

Previous record: Brasil-Lima 1998 (parasite of the Alpheus heterochaelis, A. normanni and A. viridari).

\section{Chaetiliidae Dana, 1849}

Chiriscus giambiagiae (Torti and Bastida, 1972)

Previous record: Alves and Pezzuto 2009 (cited as Macrochiridothea giambiagiae).

\section{Cirolanidae Dana, 1852}

Bathynomus giganteus A. Milne-Edwards, 1879

Specimens in a collection: $29^{\circ} 06.18^{\prime} \mathrm{S}, 45^{\circ} 51.08^{\prime} \mathrm{W}$, CEPSUL 135; 2905.96’ S, 4745.60' W, $560 \mathrm{~m}$ depth, CEPSUL 202. Previous record: Soto and Mincarone 2001.

Bathynomus miyarei Lemos de Castro, 1978

Specimens in a collection: $27^{\circ} 46.68^{\prime} \mathrm{S}, 47^{\circ} 05.66^{\prime} \mathrm{W}, 500 \mathrm{~m}$ depth, CEPSUL 131, 132, 133. Previous records: Loyola e Silva 1999; Soto and Mincarone 2001.

Excirolana armata (Dana, 1852)

Previous record: Loyola and Silva 1999; Alves and Pezzuto 2009.

Excirolana braziliensis H. Richardson, 1912 
Previous record: Alves and Pezzuto 2009.

\section{Corallanidae Hansen, 1890}

\section{Lanocira kroyeri Hansen, 1890}

Specimens in a collection: Marine biological reserve Arvoredo, DZ-UFPR uncatalogued. Previous record: Loyola e Silva et al. 2003.

\section{Cymothoidae Leach, 1814}

\section{Artystone trysibia Schiodte, 1866}

Previous record: Brasil-Lima and Barros 1998.

\section{Dubioniscidae Schultz, 1995}

\section{Novamundoniscus meridionalis (Araujo and Buckup,} 1994)

Specimens in a collection: São Lourenço d'Oeste, 26²1'15.01" S, 5250'58.31" W, UFRGS 1769; Mondaí, $27^{\circ} 06^{\prime} 06.56^{\prime \prime}$ S, 5324'01.37" W, UFRGS 1767; Nova Teutônia, 2703'07.09” S, 52²2'49.86” W, UFRGS 1766, 1768. Previous record: Araujo and Buckup 1994a; Souza-Kury 1998; Araujo 1999 (cited as Phalloniscus meridionalis).

\section{Entoniscidae Kossmann, 1881}

\section{Cancrion cancrorum (Müller, 1864)}

Previous record: Brasil-Lima 1998.

Entione achaei Müller, 1871

Previous record: Brasil-Lima 1998.

\section{Entoniscus creplinii Giard and Bonnier, 1887}

Previous record: Brasil-Lima 1998 (parasite of Polyonyx gibbesi).

\section{Entoniscus porcellanae Müller, 1862}

Previous record: Müller 1862; Brasil-Lima 1998 (parasite of Porcellana sp.).

\section{Janiridae Sars, 1897}

\section{Heterias (Fritzianira) exul (Müller, 1892)}

Previous record: Müller 1892; Lemos de Castro 1977; Bowman et al. 1987; Brasil-Lima and Barros 1998; Barros et al. 2007.

\section{Philosciidae Kinahan, 1857}

\section{Atlantoscia floridana (van Name, 1940)}

Specimens in a collection: Timbó, 2649'39.82" S, 49¹6'13.56" W, UFRGS 1754; Blumenau, 265'02.28" S, 4904'28.14" W, UFRGS 1742, 1739; Nova Teutônia, 2703'07.09” S, 52²2'49.86" W, UFRGS 1758, 1759;

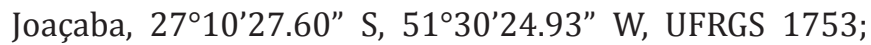
Pouso Redondo, $27^{\circ} 15^{\prime} 30.35^{\prime \prime}$ S, 49 $55^{\prime} 48.19^{\prime \prime}$ W, UFRGS 1749, 1750; Major Gercino, 2725'03.10" S, 4857'09.84" W, UFRGS 1737, 1748; São Bonifácio, 27053'57.80" S, 4855'44.06" W, UFRGS 1756, 1757; São Martinho, 2809'53.89" S, 4858'47.32" W, UFRGS 1743; Gravatal, 2819'52.41" S, 4902'08.33" W, UFRGS 1744, 1746; Urussanga, $28^{\circ} 31^{\prime} 18.85^{\prime \prime}$ S, 49¹9'14.83" W, UFRGS 1738; Tubarão, $28^{\circ} 29^{\prime} 01.98^{\prime \prime}$ S, 4900'30.90” W, UFRGS 1751,
1752; Sombrio, 2906'22.63”S, 49³8'07.22" W, UFRGS 1736, 1741; Praia Grande, 29¹1'42.68" S, 4957'09.45" W, UFRGS 1745, 1747. Previous record: Araujo et al. 1996.

Benthana picta (Brandt, 1833)

Specimens in a collection: Faxinal dos Guedes, 26 $6^{\circ} 1^{\prime} 09.71^{\prime \prime}$ S, 52 $15^{\prime} 36.32^{\prime \prime}$ W, UFRGS 1704; Ponte

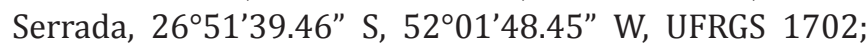
Catanduvas, $27^{\circ} 04^{\prime} 19.71^{\prime \prime} \mathrm{S}, 5^{\circ} 39^{\prime} 31.47^{\prime \prime}$ W, UFRGS 1700, 1706; Joaçaba, $27^{\circ} 10^{\prime} 27.60^{\prime \prime}$ S, 5130'24.93” W, UFRGS 1701; Campos Novos, 2724'04.89” S, 5113'23.96" W, UFRGS 1705; São Joaquim, 28¹7'32.20” S, 4956'05.48” W, UFRGS 1703. Previous record: Araujo et al. 1996; Souza-Kury 1998.

Benthana longicornis Verhoeff, 1941

Specimens in a collection: Nova Teutônia, Itá, 27¹6'36.19” S, 52¹9'55.24" W, SMNH; Nova Teutônia, 2703'07.09" S, 52²2'49.86" W, UFRGS 1505, 1506. Previous record: Andersson 1960; Araujo et al. 1996; Souza-Kury 1998.

Benthana taeniata Araujo and Buckup, 1994

Specimens in a collection: Papanduva, 26 24'25.71" S, 5008'32.52" W, UFRGS 1507, 1510. Previous record: Souza-Kury 1998; Araujo 1999.

Burmoniscus meeusei (Holthuis, 1947)

Specimens in a collection: Timbó, $26^{\circ} 49^{\prime} 39.82^{\prime \prime} \mathrm{S}$, 4916'13.56" W, UFRGS 1501; Blumenau, 2655'02.28" S, 4904'28.14" W, UFRGS 1502; Joaçaba, 27²10'27.60" S, 5130'24.93" W, UFRGS 1503; Major Gercino, 27²5'03.10" S, 48 57'09.84" W, UFRGS 1504. Previous record: Araujo et al. 1996; Souza-Kury 1998.

\section{Platyarthridae Vandel, 1946}

\section{Trichorhina acuta Araujo and Buckup, 1994}

Specimens in a collection: Itapoá, 26 $07^{\circ} 02.72^{\prime \prime}$ S, 48³7'00.15" W, UFRGS 1882; Pouso Redondo, 2715'30.35" S, 4955'48.19” W, UFRGS 1876; São Bonifácio, 2753'57.80” S, 4855'44.06” W, UFRGS 1878. Previous record: Araujo and Buckup 1994b; Souza-Kury 1998; Araujo 1999.

\section{Trichorhina argentina Vandel, 1963}

Specimens in a collection: Joaçaba, 2710'27.60" S, 5130'24.93" W, UFRGS 1517; Sombrio, 2906'22.63" S, 49³8'07.22" W, UFRGS 1518. Previous record: Araujo and Buckup 1996b; Araujo 1999.

\section{Trichorhina tatianae Araujo and Almerão, 2007}

Specimens in a collection: Morro dos Conventos, Araranguá, 2856'29.65” S, 49²9'01.06" W, MNRJ 19736; Morro dos Conventos, Araranguá, 2856'29.65” S, 49²9’01.06” W, UFRGS 4011. Previous record: Araujo and Almerão 2007.

Trichorhina tomentosa (Budde-Lund, 1893)

Specimens in a collection: Blumenau, 26 55'02.28" S, 4904'28.14" W, UFRGS 1995, 1996; Pouso Redondo, 2715'30.35” S, 4955'48.19” W, UFRGS 1998; Tubarão, $28^{\circ} 29^{\prime} 01.98^{\prime \prime}$ S, 4900'30.90” W, UFRGS 1999; Sombrio, 2906'22.63" S, 49³8'07.22" W, UFRGS 2001. Previous record: Araujo and Buckup 1996a.

Trichorhina bicolor Araujo and Buckup, 1996

Specimens in a collection: São Bonifácio, 2753'57.80" S, 4855'44.06" W, UFRGS 2004; Pouso Redondo, $27^{\circ} 15^{\prime} 30.35^{\prime \prime}$ S, 4955'48.19” W, UFRGS 2005; Tubarão, $28^{\circ} 29^{\prime} 01.98^{\prime \prime}$ S, 4900’30.90” W, UFRGS 2003. Previous 
record: Araujo and Buckup 1996a.

\section{Trichorhina brasilensis Andersson, 1960}

Specimens in a collection: Nova Teutônia, Itá, 27¹6'36.19" S, 52¹9'55.24" W, SMNH. Previous record: Andersson 1960; Souza-Kury 1998.

\section{Porcellionidae Brandt, 1831}

\section{Porcellio dilatatus Brandt, 1833}

Specimens in a collection: Papanduva, 26²4'25.71" S, 5008'32.52" W, UFRGS 1676; São Lourenço d'Oeste, 2621'15.01" S, 5250'58.31" W, UFRGS 1668; Xanxerê,

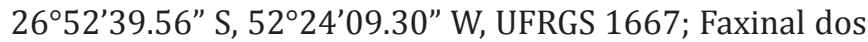

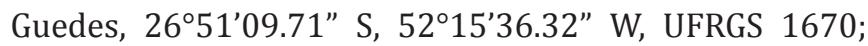

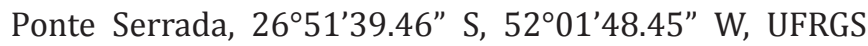
1672; Irani, $27^{\circ} 01^{\prime} 15.38^{\prime \prime}$ S, 51 $53^{\circ} 04.68^{\prime \prime}$ W, UFRGS 1775; Santa Cecília, 2657'54.03" S, 50²5'11.39" W, UFRGS 1669; Joaçaba, $27^{\circ} 10^{\prime} 27.60^{\prime \prime}$ S, 5130'24.93" W, UFRGS 1671; Itá, $27^{\circ} 16^{\prime} 36.19^{\prime \prime}$ S, 52¹9'55.24" W, UFRGS 1666; Campos Novos, $27^{\circ} 24^{\prime} 04.89^{\prime \prime}$ S, 5113'23.96” W, UFRGS 1677; Urubici, $28^{\circ} 00^{\prime} 15.23^{\prime \prime} \mathrm{S}, 4^{\circ} 35^{\prime} 17.55^{\prime \prime} \mathrm{W}$, UFRGS 1673; São Joaquim, 28¹7'32.20" S, 4956’05.48” W, UFRGS 1674. Previous record: Araujo et al. 1996; SouzaKury 1998. Note: Exotic species.

\section{Porcellio scaber Latreille, 1804}

Specimens in a collection: Bom Retiro, 27 ${ }^{\circ} 47^{\prime} 57.51^{\prime \prime} \mathrm{S}$, 49²9'22.04" W, UFRGS 1697. Previous record: Araujo et al. 1996; Souza-Kury 1998. Note: Exotic species.

\section{Porcellio laevis Latreille, 1804}

Specimens in a collection: Papanduva, 26 24'25.71" S, 5008'32.52" W, UFRGS 1690; Campos Novos, 27²4'04.89" S, 5113'23.96" W, UFRGS 1691. Previous record: Araujo et al. 1996. Note: Exotic species.

\section{Porcellionides pruinosus (Brandt, 1833)}

Specimens in a collection: Papanduva, 26²4'25.71" S, 5008'32.52" W, UFRGS 1636; São Lourenço d'Oeste, 26²1'15.01" S, 5250'58.31" W, UFRGS 1637; Anchieta, 26³2'07.61" S, 5319'48.45" W, UFRGS 1629; São Miguel d'Oeste, 2643'52.85" S, 5331'08.57" W, UFRGS 1630;

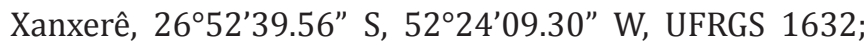
Faxinal dos Guedes, 26 51'09.71" S, 52 $2^{\circ} 15^{\prime} 36.32^{\prime \prime}$ W, UFRGS 1635; Ponte Serrada, 2651'39.46" S, 5201'48.45" W, UFRGS 1640; Timbó, 2649’39.82" S, 49¹6'13.56" W, UFRGS 1631; Chapecó, 2705'48.43" S, 52³7’06.42" W,

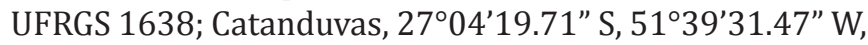
UFRGS 1627; Nova Teutônia, 2703'07.09" S, 52²2'49.86"

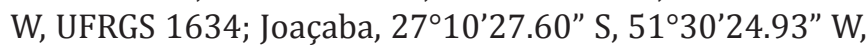
UFRGS 1626; Pouso Redondo, 27¹5'30.35" S, 4955'48.19" W, UFRGS 1633; Itá, 27¹6’36.19”S, 52¹9'55.24" W, UFRGS 1628; Campos Novos, 2724'04.89” S, 51¹3'23.96" W, UFRGS 1642; Major Gercino, 27²5'03.10" S, 48 ${ }^{\circ} 7^{\prime} 09.84^{\prime \prime}$ W, UFRGS 1641; São Bonifácio, 2753'57.80" S, 4855'44.06" W, UFRGS 1643; Sombrio, 2906'22.63" S, 49³8'07.22" W, UFRGS 1639; Nova Teutônia, Itá, 2703'07.09” S, 52²2'49.86” W, SMNH. Previous records: Andersson 1960; Araujo et al. 1996; Souza-Kury 1998. Note: Exotic species.

Porcellionides sexfasciatus (Koch, 1847)

Specimens in a collection: Papanduva, 26 $24^{\prime} 25.71^{\prime \prime} \mathrm{S}$, 5008'32.52" W, UFRGS 1647; Joaçaba, 27¹0'27.60" S, 5130'24.93" W, UFRGS 1648; Campos Novos, 2724'04.89" S, 51¹3'23.96" W, UFRGS 1649; Bom Jardim da Serra,
28²0'19.97” S, 49³7’30.87” W, UFRGS 1650. Previous record: Araujo et al. 1996; Souza-Kury 1998; Araujo 1999. Note: Exotic species.

\section{Sphaeromatidae Latreille, 1825}

Cassidinidea fluminensis (Mañe-Garzón, 1944)

Previous record: Loyola e Silva 1999.

Dynamenella tropica Loyola e Silva, 1960

Previous record: Loyola e Silva 1999.

Pseudophaeroma jakobii Loyola e Silva, 1959

Previous record: Loyola e Silva 1999.

Sphaeromopsis mourei (Loyola e Silva, 1960)

Previous record: Loyola e Silva 1999.

\section{Styloniscidae Vandel, 1952}

\section{Pectenoniscus angulatus Andersson, 1960}

Specimens in a collection: Nova Teutônia, Itá, 27¹6 36.19” S, 52 19'55.24" W, SMNH. Previous record: Andersson 1960; Souza-Kury 1998.

\section{Trachelipodidae Strouhal, 1953}

Nagurus cristatus (Dollfus, 1889)

Specimens in a collection: Nova Teutônia, 2703'07.09" S, 52²2'49.86" W, UFRGS 2077; Pouso Redondo, $27^{\circ} 15^{\prime} 30.35^{\prime \prime}$ S, 495 $55^{\prime} 48.19^{\prime \prime}$ W, UFRGS 2075; Major Gercino, 27²5'03.10” S, 4857'09.84” W, UFRGS 2074; Gravatal, 28¹9'52.41” S, 4902'08.33” W, UFRGS 2076. Previous record: Araujo and Buckup 1996b.

Nagurus nanus (Budde-Lund, 1908)

Specimens in a collection: Gravatal, 28 $8^{\circ} 19^{\prime} 52.41^{\prime \prime} \mathrm{S}$, 4902'08.33" W, UFRGS 2078. Previous record: Araujo and Buckup 1996b.

\section{Mysida Haworth, 1825}

Mysidae Haworth, 1825

Chlamydopleon dissimile (Coifmann, 1937)

Previous record: Alves and Pezzuto 2009 (cited as Bowmaniella brasiliensis).

\section{Stomatopoda Latreille, 1817}

\section{Lysiosquillidae Giesbrecht, 1910}

\section{Lysiosquilla scabricauda (Lamarck, 1818)}

Specimens ina a collection: $26^{\circ} 31.52^{\prime} \mathrm{S}, 48^{\circ} 19.84^{\prime} \mathrm{W}, 40 \mathrm{~m}$ depth, CEPSUL 59; Barra Sul, Florianópolis, UFRGS 3409. Previous record: Gomes-Corrêa 1998; Tavares 2002a.

\section{Hemisquillidae Manning, 1980}

Hemisquilla braziliensis (Moreira, 1903)

Specimens in a collection: Ponta das Canas, Florianópolis, UFRGS 3462. Previous record: Gomes-Corrêa 1998; 1999.

Nannosquillidae Manning, 1980

Coronis scolopendra Latreille, 1828 
Previous record: Gomes-Corrêa 1998; 1999.

Squillidae Latreille, 1802

Cloridopsis dubia (A. Milne-Edwards, 1837)

Previous record: Gomes-Corrêa 1998.

Gibbesia prasinolineata (Dana, 1852)

Previous record: Gomes-Corrêa 1998 (cited as Squilla prasinolineata).

Squilla brasiliensis Calman, 1917

Specimens in a collection: $26^{\circ} 48.72^{\prime} \mathrm{S}, 46^{\circ} 44.99^{\prime} \mathrm{W}, 206 \mathrm{~m}$ depth, CEPSUL 191. Previous record: Gomes-Corrêa 1998; 1999.

Squilla grenadensis Manning, 1969

Previous record: Gomes-Corrêa 1998; Branco and Fracasso 2004; Rodrigues and Young 2005; Freitas Jr. et al. 2010.

Squilla neglecta Gibbes, 1850

Specimens in a collection: Porto Belo, UFRGS 3363.

Previous record: Gomes-Corrêa 1998; 1999.

Maxillopoda Dahl, 1956

Arguloidea Yamaguti, 1963

Argulidae Yamaguti, 1963

Argulus spinulosus Silva, 1980

Previous record: Malta 1998.

Calanoida G. O. Sars, 1903

Acartiidae Sars, 1900

Acartia (Acartia) danae Giesbrecht, 1889

Previous record: Campaner 1985.

Acartia lilljeborgii Giesbrecht, 1889

Previous record: Veado and Resgalla Jr. 2005; Resgalla Jr. et al. 2008; Veado et al. 2010.

Acartia tonsa Dana, 1849

Previous record: Veado and Resgalla Jr. 2005; Veado et al. 2010.

\section{Aetideidae Giesbrecht, 1892}

Paivella naporai Wheeler, 1970

Previous record: Campaner 1985.

Augaptilidae Sars G.0., 1905

Haloptilus longicornis (Claus, 1863)

Previous record: Campaner 1985.

Calanidae Dana, 1849

Calanoides carinatus (Kroyer, 1849)

Previous record: Campaner 1985.

Calocalanus pavo (Dana, 1852)

Previous record: Campaner 1985.

Mesocalanus tenuicornis (Dana, 1849)

Previous record: Campaner 1985 (cited as Calanus tenuicornis).

Nannocalanus minor (Claus, 1863)
Previous record: Campaner 1985 (cited as Calanus minor). Undinula vulgaris (Dana, 1849)

Previous record: Campaner 1985.

Candaciidae Giesbrecht, 1893

Candacia curta (Dana, 1849)

Previous record: Campaner 1985.

Candacia pachydactyla (Dana, 1849)

Previous record: Campaner 1985.

Centropagidae Giesbrecht, 1892

Centropages velificatus (Oliveira, 1947)

Previous record: Campaner 1985; Veado and Resgalla Jr. 2005; Veado et al. 2010.

Clausocalanidae Giesbrecht, 1893

Clausocalanus furcatus (Brady, 1883)

Previous record: Campaner 1985.

Clausocalanus arcuicornis (Dana, 1849)

Previous record: Campaner 1985.

Clausocalanus parapergens Frost \& Fleminger, 1968

Previous record: Campaner 1985.

Ctenocalanus vanus Giesbrecht, 1888

Previous record: Campaner 1985.

Diaptomidae Baird, 1850

Notodiaptomus henseni (Dahl, 1894)

Previous record: Santos-Silva 1998.

Eudiaptomus vulgaris (Schmeil, 1896)

Previous record: Veado et al. 2010.

Eucalanidae Giesbrecht, 1893

Subeucalanus pileatus (Giesbrecht, 1888)

Previous record: Campaner 1985 (cited as Eucalanus pileatus); Veado and Resgalla Jr. 2005 (cited as Eucalanus pileatus).

Pareucalanus sewelli (Fleminger, 1973)

Previous record: Campaner 1985 (cited as Eucalanus sewelli).

Euchaeta marina (Prestandrea, 1833)

Previous record: Campaner 1985.

Heterorhabdidae Sars G.0., 1902

Heterorhabdus papilliger (Claus, 1863)

Previous record: Campaner 1985.

Lucicutiidae Sars G.0., 1902

Lucicutia flavicornis (Claus, 1863)

Previous record: Campaner 1985.

Paracalanidae Giesbrecht, 1893

Paracalanus aculeatus Giesbrecht, 1888

Previous record: Campaner 1985.

Paracalanus indicus Wolfenden, 1905 
Previous record: Campaner 1985.

Paracalanus nanus (G. O. Sars, 1907)

Previous record: Veado and Resgalla Jr. 2005.

Paracalanus quasimodo Bowman, 1971

Previous record: Veado and Resgalla Jr. 2005; Resgalla Jr. et al. 2008; Veado et al. 2010.

Parvocalanus crassirostris (F. Dahl, 1894)

Previous record: Veado and Resgalla Jr. 2005; Resgalla Jr. et al. 2008; Veado et al. 2010.

\section{Phaennidae Sars, 1902}

Phaenna spinifera Claus, 1863

Previous record: Veado and Resgalla Jr. 2005.

Xanthocalanus marlyae Campaner, 1978

Previous record: Campaner 1985.

Pontellidae Dana, 1852

\section{Calanopia americana F. Dahl, 1894}

Previous record: Veado and Resgalla Jr. 2005; Veado et al. 2010.

Labidocera fluviatilis F. Dahl, 1894

Previous record: Resgalla Jr. et al. 2008; Veado et al. 2010.

Labidocera nerii (Krøyer, 1849)

Previous record: Veado et al. 2010.

\section{Pseudodiaptomidae Sars, 1902}

Pseudodiaptomus acutus (Dahl F., 1894)

Previous record: Veado et al. 2010.

Pseudodiaptomus richardi (Dahl F., 1894)

Previous record: Veado and Resgalla Jr. 2005; Veado et al. 2010.

\section{Scolecitrichidae Giesbrecht, 1893}

Amallothrix tenuiserrata (Giesbrecht, 1893)

Previous record: Campaner 1985 (cited as Scolecithricella tenuiserrata).

Scolecithrix danae (Lubbock, 1856)

Previous record: Campaner 1985.

\section{Temoridae Giesbrecht, 1893}

\section{Temora stylifera (Dana, 1849)}

Previous record: Campaner 1985; Veado and Resgalla Jr. 2005; Resgalla Jr. et al. 2008; Veado et al. 2010.

Temora turbinata (Dana, 1849)

Previous record: Resgala Jr. 2001; Veado and Resgalla Jr. 2005; Resgalla Jr. et al. 2008; Veado et al. 2010. Note: Exotic species.

\section{Cyclopoida Burmeister, 1834}

Cyclopidae Dana, 1846

Acanthocyclops michaelseni (Mrazek, 1901)

Specimens in a collection: Rio Espingarda, Nacional Park of the Serra do Itajaí, $27^{\circ} 01^{\prime} 25.97^{\prime \prime} \mathrm{S}, 49^{\circ} 09^{\prime} 08.71^{\prime \prime} \mathrm{W}$, UFRGS 3574.
Ectocyclops phaleratus (Koch, 1838)

Previous record: Rocha and Botelho 1998.

Eucyclops delachauxi (Kiefer, 1925)

Previous record: Veado et al. 2010.

Eucyclops serrulatus (Fischer, 1851)

Previous record: Rocha and Botelho 1998.

Macrocyclops albidus (Jurine, 1820)

Previous record: Rocha and Botelho 1998; Veado et al. 2010.

Megacyclops viridis (Jurine, 1820)

Previous record: Veado et al. 2010.

Mesocyclops aspericornis (Daday, 1906)

Previous record: Veado et al. 2010.

Mesocyclops leuckarti (Claus, 1857)

Previous record: Rocha and Botelho 1998.

Mesocyclops longisetus (Thiébaud, 1912)

Previous record: Veado et al. 2010.

Mesocyclops meridianus (Kiefer, 1926)

Previous record: Serafim-Júnior et al. 2006; Veado et al. 2010.

Metacyclops brauni Herbst, 1962

Previous record: Veado et al. 2010.

Metacyclops mendocinus (Wierzejski, 1892)

Previous record: Veado et al. 2010.

Microcyclops anceps (Richard, 1897)

Previous record: Serafim-Júnior et al. 2006; Veado et al. 2010.

Microcyclops ceibaensis (Marsh, 1919)

Previous record: Serafim-Júnior et al. 2006; Veado et al. 2010.

Microcyclops finitimus Dussart, 1984

Previous record: Veado et al. 2010.

Paracyclops chiltoni (G. M. Thomson, 1883)

Previous record: Serafim-Júnior et al. 2006.

Thermocyclops brehmi (Kiefer, 1927)

Previous record: Veado et al. 2010.

Thermocyclops decipiens (Kiefer, 1929)

Previous record: Veado et al. 2010.

Tropocyclops prasinus (Fischer, 1860)

Specimens in a collection: Rio Garcia, Nacional Park of the Serra do Itajaí, $27^{\circ} 03^{\prime} 38.99^{\prime \prime}$ S, 4906'43.50” W, UFRGS 3576; Rio Garrafa, Nacional Park of the Serra do Itajaí, $27^{\circ} 03^{\prime} 48.42^{\prime \prime} \mathrm{S}, 4^{\circ} 05^{\prime} 42.26^{\prime \prime} \mathrm{W}$, UFRGS 3573. Previous record: Serafim-Júnior et al. 2006; Veado et al. 2010.

Tropocyclops schubarti Kiefer, 1935

Previous record: Veado et al. 2010.

Oithonidae Dana, 1853

Oithona oswaldocruzi Oliveira, 1945

Previous record: Rocha and Botelho 1998; Veado and Resgalla Jr. 2005; Resgalla Jr. et al. 2008.

Oithona ovalis Herbst, 1955

Previous record: Resgalla Jr. et al. 2008; Veado et al. 2010. Oithona plumifera Baird, 1843

Previous record: Rocha and Botelho 1998; Veado and Resgalla Jr. 2005; Resgalla Jr. et al. 2008; Veado et al. 2010.

Oithona robusta Giesbrecht, 1891

Previous record: Campaner 1985.

Oithona setigera Dana, 1852 
Previous record: Campaner 1985.

Harpacticoida G. O. Sars, 1903

Aegisthidae Giesbrecht, 1893

Aegisthus mucronatus Giesbrecht, 1891

Previous record: Reid 1998.

Clytemnestridae Scott, 1909

Clytemnestra rostrata (Brady, 1883)

Previous record: Reid 1998; Resgalla Jr. et al. 2008.

Clytemnestra scutellata Dana, 1849

Previous record: Reid 1998.

Diosaccidae G. O. Sars, 1906

Robertsonia mourei M. H Nogueira, 1961

Previous record: Reid 1998.

Ectinosomatidae Sars, 1903

Ectinosoma dentatum Steuer, 1940

Previous record: Reid 1998.

Microsetella norvegica (Boeck, 1865)

Previous record: Reid 1998.

Microsetella rosea (Dana, 1848)

Previous record: Veado et al. 2010.

Euterpinidae Brian, 1921

Euterpina acutifrons (Dana, 1849)

Previous record: Reid 1998; Veado and Resgalla Jr. 2005;

Resgalla et al. 2008; Veado et al. 2010.

Harpacticidae Dana, 1846

Harpacticus poppei Richard, 1897

Previous record: Reid 1998.

Laophontidae Scott, 1905

Heterolaophonte hamata Jakobi, 1954

Previous record: Reid 1998.

Heterolaophonte manifera (C. B. Wilson, 1931)

Previous record: Reid 1998.

Loureirophonte catharinensis Jakobi, 1953

Previous record: Reid 1998.

Loureirophonte paranaensis Jakobi, 1953

Previous record: Reid 1998.

Mourephonte longiset (Nicholls, 1941)

Previous record: Reid 1998.

Paralaophonte lacerdai Jakobi, 1953

Previous record: Reid 1998.

Onychocamptus besnardi Jakobi, 1954

Previous record: Reid 1998.

Louriniidae Monard, 1927

Lourinia armata (Claus, 1866)

Previous record: Reid 1998.
Miraciidae Dana, 1846

Macrosetella gracilis (Dana, 1847)

Previous record: Reid 1998; Veado and Resgalla Jr. 2005;

Resgalla et al. 2008.

Miracia efferata Dana, 1849

Previous record: Reid 1998.

Orthopsyllidae Huys, 1990

Orthopsyllus improportionatus (Jakobi, 1954)

Previous record: Reid 1998.

Parastenocarididae Chappuis, 1933

Parastenocaris brasilibathynellae Jakobi and Loyola e Silva, 1962

Previous record: Reid 1998.

Parastenocaris hurdi Jakobi and Loyola e Silva, 1962

Previous record: Reid 1998.

Tegastidae G. O. Sars, 1904

Parategastes herteli Jakobi, 1953

Previous record: Reid 1998.

Parategastes sphaericus punicus Monard, 1935

Previous record: Reid 1998.

Tegastes brasiliensis Jakobi, 1953

Previous record: Reid 1998.

Tisbidae Stebbing, 1910

Scutellidium longicauda paranaense Steudel, 1970

Previous record: Reid 1998.

Scutellidium loureiroi Jakobi, 1954

Previous record: Reid 1998.

Thalestridae G. 0. Sars, 1905

Diarthrodes falcipes Marinoni, 1964

Previous record: Reid 1998.

Kentrogonida Delage, 1884

Lernaeodiscidae Boschma, 1928

Lernaeodiscus porcellanae Müller, 1862

Previous record: Young 1998 (Parasite of the porcellanid crustaceans Petrolisthes galathinus, P. cabrilloi, and $P$. armatus).

Peltogastridae Lilljeborg, 1860

Peltogaster purpureus (Müller, 1862)

Previous record: Young 1998 (Parasite of an unidentified species of Paguroidea).

Peltogastrella sulcata (Lilljeborg, 1859)

Previous record: Young 1998 (cited as Peltogaster socialis).

Lepadiformes Buckeridge and Newman, 2006

Lepadidae Darwin, 1852 
Lepas anatifera Linnaeus, 1758

Previous record: Young 1998; 1999.

Lepas anserifera Linnaeus, 1767

Previous record: Young 1998; 1999.

Dosima fascicularis (Ellis and Solander, 1786)

Previous record: Young 1998; 1999.

Poecilasmatidae Annandale, 1909

Poecilasma inaequilaterale Pilsbry, 1907

Previous record: Young 1998.

Poecilostomatoida Thorell, 1859

Clausidiidae Embleton, 1901

Hemicyclops thalassius Vervoort and Ramírez, 1966

Previous record: Veado and Resgalla Jr. 2005.

Corycaeidae Dana, 1852

Corycaeus amazonicus Dahl F., 1894

Previous record: Campaner 1985.

Corycaeus giesbrechti F. Dahl, 1894

Previous record: Campaner 1985.

Corycaeus speciosus Dana, 1849

Previous record: Campaner 1985.

Corycaeus typicus (Kroyer, 1849)

Previous record: Campaner 1985.

Farranula gracilis (Dana, 1849)

Previous record: Campaner 1985.

Oncaeidae Giesbrecht, 1893

Oncaea venusta Philippi, 1843

Previous record: Campaner 1985; Veado and Resgalla Jr. 2005.

Sapphirinidae Thorell, 1859

Copilia mirabilis Dana, 1852

Previous record: Campaner 1985.

Scalpelliformes Buckeridge and Newman, 2006

Scalpellidae Pilsbry, 1907

Arcoscalpellum boubalocerus Young, 1992

Previous record: Young 1998; 1999.

Hamatoscalpellum rathbunae (Pilsbry, 1907)

Previous record: Young 1998.

Litoscalpellum henriquecostai (Weber, 1960)

Previous record: Young 1998; 1999.

Octolasmis lowei (Darwin, 1851)

Previous record: Young 1998; 1999.

Siphonostomatoida Thorell, 1859

Lernaeopodidae H. Milne Edwards, 1840

Brachiella thynni Cuvier, 1830
Previous record: Ho 1998; Luque and Tavares 2007

(parasitic on the fins of Acanthocybium solandri,

Argyrosomus regium, Pomatomus saltarix, Sciaena aquila,

Scomberomorus cavalla, $S$. regalis, Thunnus albacares, $T$.

obesus, and T. thynnus).

Minilernaea floricapitella Thatcher and Huergo, 2005

Previous record: Luque and Tavares 2007; Thatcher and Huergo 2005 (parasitic on the body surface and near fins of Astyanax sp., Corydoras ehrhardti).

Pandaridae H. Milne Edwards, 1840

Pandarus floridanus Cressey, 1967

Previous record: Luque and Tavares 2007 (parasitic on the body surface of Prionace glauca).

Pandarus satyrus Dana, 1849

Previous record: Luque and Tavares 2007 (parasitic on the body surface of Prionace glauca).

Sessilia Lamarck, 1818

Archaeobalanidae Newman and Ross, 1976

Conopea galeata (Linnaeus, 1771)

Previous record: Young 1998.

Balanidae Leach, 1817

Balanus amphitrite Darwin, 1854

Previous record: Young 1998; 1999.

Balanus improvisus Darwin, 1854

Previous record: Young 1998; 1999.

Balanus spongicola Brown, 1844

Previous record: Young 1998; 1999.

Balanus trigonus Darwin, 1854

Previous record: Young 1998; 1999.

Balanus venustu Darwin, 1854

Previous record: Young 1998; 1999.

Fistulobalanus citerosum (Henry, 1974)

Previous record: Young 1998; 1999.

Megabalanus coccopoma (Darwin, 1854)

Previous record: Young 1998; 1999; Severino and Resgalla Jr. 2005. Note: Exotic species.

Megabalanus tintinnabulum (Linnaeus, 1758)

Specimens in a collection: Marissol beach, Imbituba, UFRGS 3464. Previous record: Young 1998; 1999.

Megabalanus vesiculosus Darwin, 1854Previous record: Young 1998.

Chthamalidae Darwin, 1854

Chthamalus bisinuatus Pilsbry, 1916

Previous record: Young 1998, 1999.

Chthamalus proteus Dando and Southward, 1980

Previous record: Young 1998.

Chelonibiidae Pilsbry, 1916

Chelonibia patula (Ranzani, 1818)

Previous record: Young 1998.

Chelonibia testudinaria (Linnaeus, 1757) 
Previous record: Young 1998; 1999.

\section{Tetraclitidae Gruvel, 1903}

Tetraclita stalactifera (Lamarck, 1818)

Previous record: Young 1998; 1999.

Ostracoda Latreille, 1802

\section{Podocopida Sars, 1866}

\section{Cytherideidae Sars, 1925}

Cyprideis multidentata Hartmann, 1955

Previous record: Martens et al. 1998.

Cytherura purperae Ornellas and Fallavena, 1978

Previous record: Martens et al. 1998.

Perissocytheridae krommelbeini Pinto and Ornellas, 1970

Previous record: Martens et al. 1998.

\section{Darwinulidae Brady and Norman, 1889}

\section{Darwinula serricaudata espinosa Pinto and Kotzian,} 1961

Specimens in a collection: Rio Espingarda, Nacional Park of the Serra do Itajaí, $27^{\circ} 01^{\prime} 25.97^{\prime \prime} \mathrm{S}, 4^{\circ} 09^{\prime} 08.71^{\prime \prime} \mathrm{W}$, UFRGS 3578.

\section{Eucandonidae Swain, 1961}

\section{Pseudocandona pumilis Würdig and Pinto, 1999}

Specimens in a collection: Rio Garcia, Nacional Park of the Serra do Itajaí, $27^{\circ} 03^{\prime} 38.99^{\prime \prime}$ S, 4906'43.50” W, UFRGS 3577; Rio Espingarda, Nacional Park of the Serra do Itajaí, $27^{\circ} 01^{\prime} 25.97^{\prime \prime}$ S, 4909'08.71” W, UFRGS 3579.

\section{Limnocytheridae Klie, 1938}

\section{Elpidium bromeliarum Müller, 1880}

Previous record: Müller, 1880b; Martens et al. 1998.

\section{Neocytherideidae Puri, 1957}

\section{Minicythere heinii Ornellas, 1974}

Previous record: Martens et al. 1998.

For the first time, the following 19 species were recorded in Santa Catarina: Acanthocyclops michaelseni (Mrazek, 1901); Pseudocandona pumilis Würdig and Pinto, 1999; Darwinula serricaudata espinosa Pinto and Kotzian, 1961; Aegla brevipalma Bond-Buckup and Santos, 2012; A. camargoi Buckup and Rossi, 1977; A. leachi Bond-Buckup and Santos, 2012; A. leptodactyla Bond-Buckup and Buckup, 1994; A. oblata Bond-Buckup and Santos, 2012; A. serrana Buckup and Rossi, 1977; A. singularis Ringuelet, 1948; Alpheus bouvieri A. Milne-Edwards, 1878; Panulirus laevicauda (Latreille, 1817); Plesionika edwardsii (Brandt, 1851); Latreutes parvulus (Stimpson, 1866); Uca burgesi Holthuis, 1967; Uca mordax (Smith, 1870); Pilumnus quoii H. Milne Edwards, 1834; Megalobrachium roseum (Rathbun, 1900) and Rochinia crassa (A. Milne-Edwards, 1879) .
Decapoda was the largest order with 280 species listed, that corresponds to about $50 \%$ of decapod species known in Brazil (Amaral and Jablonski 2005).

Regarding to the richness of marine decapods, the coast of Santa Catarina (25ㅇ' 29 $^{\circ} 19^{\prime}$ S), situated to the north of the Santa Marta Grande Cape (28 $\left.40^{\prime} \mathrm{S}\right)$, is characterized by the influence of the Brazil current, that carries tropical water $\left(>20^{\circ} \mathrm{C}\right)$, by the supply of freshwater and by the South Central Atlantic water $\left(<20^{\circ} \mathrm{C}\right)$. The south of the Santa Marta Grande Cape is influenced mainly by the Subtropical Convergence between the Brazil and Malvinas currents, with seasonal variations in distribution of water masses (Emilson 1961; Carvalho et al. 1998; Amaral and Jablonski 2005). Futhermore the coast of Santa Catarina is under influence of tropical and temperate climates, a transition condition that favors the richness of Crustacea species in the region. This condition is also observed in the austral limit of mangrove in Brazilian coast, located in the south of Santa Catarina (Schaeffer-Novelli et al. 1990).

Following the classification provided by Melo (1985), in terms of latitudinal distribution, the categories of species were recorded for the Brachyura (Decapoda) listed in this study: amphi-American (e.g. Aratus pisonii, Pilumnus reticulates, Pyromaia tuberculata), amphi-Atlantic (e.g. Moreiradromia antillensis, Apiomithrax violaceus, Callapa galloides, Cataleptodius floridanus, Goniopsis cruentata, Hepatus pudibundus, Homola barbata, Menippe nodifrons, Pachygrapsus gracilis), circumtropical (e.g. Cronius ruber, Ovalipes trimaculatus, Cyclograpsus integer, Pachygrapsus transversus, Planes cyaneus) and Western Atlantic (e.g. Holothuriophilus tomentosus, Pilumnoides coelhoi, Trichopeltarion pezzutoi).

Likewise, brachyuran species were observed from different zoogeographic provinces (Melo 2010; Bochi 2000; Bochi and Gavio 2005): Magellan (e.g. Collodes rostratus, Corystoides chilensis, Leurocyclus tuberculosus, Eurypodius latreillei), Argentinian (e.g. Libinia spinosa, Pelia rotunda, Podochela atlantica, Rochinia gracilipes, Spinolambrus fraterculus), Carolinian (e.g. Achelous spinicarpus, Callinectes danae, Hexapanopeus paulensis, Mesorhea sexspinosa, Microphrys bicornutus, Stenorhynchus seticornis) and Virginian (e.g. Achelous spinimanus, Arenaeus cribrarius, Callinectes ornatus, Persephona mediterranea, Spinolambrus pourtalesii).

It's highlighted the fact that the continental shelf of Santa Catarina state has been considered as part of the Argentinian zoogeographic province due to the estuaries influence, especially Lagoa dos Patos and Río de La Plata, that consequently influences the distribution of decapod species, besides the interaction between the Malvinas and Brazil Current, in the outer shelf and slope (Bochi 2000).

The families of Decapoda with the greatest number of species recorded were: Aeglidae (19), Palaemonidae (13) and Portunidae (13).

From the total of 19 species of the genus Aegla present in the Santa Catarina state, seven were cited for the first time. These species represent nearly $50 \%$ of known species of the genus in Brazil (Bond-Buckup 2003, Bond-Buckup 2010). The species inhabits exclusively rivers and streams with clean and well oxygenated waters and are considered good indicators of environmental quality (Bond-Buckup, 2003). However, the increasing deterioration of these 
environments has threatened several species. In a recent assessment of the crustaceans' conservation in Brazil (Magris et al. 2010), 12 species of this genus have been evaluated and categorized as threatened. Therefore, the information about distributions that this checklist refers to is an important contribution to assessing the risk of extinction of these species and also for environmental quality assessment in watersheds where they occur.

It is important to cite that some records of exotic species were also evidenced: Armadillidium vulgare, Porcellio dilatatus, Porcellio scaber, Porcellio laevis, Porcellionides pruinosus, Porcellionides sexfasciatus, Temora turbinata, Megabalanus coccopoma and Charybdis hellerii. Among the exotic species, terrestrial Isopoda showed the highest number of species recorded (6), with original distribution from Europe, following the human presence in the field or in towns (Leistikow and Wagele 1999).

In conclusion, Santa Catarina state constitutes a particular region for the study of Crustacea in the land, rivers or sea. This can be observed by the presence of terrestrial Isopoda, an important part of nutrient cycling and soil formation (Quadros and Araujo 2008), by the diversity of Aeglidae in streams and, also, by the special oceanographic conditions, that make possible the occurrence of decapods with different environmental requirements or origin.

ACKNOWLEDGMENTS: Support for this project was supplied by Instituto Chico Mendes de Conservação da Biodiversidade - ICMBio (Projeto de Curadoria e Manutenção da Coleção Científica do CEPSUL) to HB and RAS. Additional support come from by Fundação de Amparo à Pesquisa do Estado de São Paulo - FAPESP (Biota 2010/50188-8; Coleções Cientificas 2009/54931-0) to FLM, by Conselho Nacional de Desenvolvimento Científico e Tecnológico - CNPq (Research Grants Proc. No. 472746/2004-9, 473050/2007-2 and 471011/2011-8 to FLM; Projeto PPBio Proc. N ${ }^{\circ} 505156 / 2007-5$ to INPA; Research Fellowships 302748/2010-6 to FLM; 304468/2009-6 to CM; 302274/2011-1 to PBA) and Coordenação de Aperfeiçoamento de Pessoal de Nível Superior CAPES (PNPD 23038.007529/2011-93 to MPA). The authors also would like to thank Jorge Eduardo Kotas, Eloisa Pinto Vizuete and Luciana da Cunha for reviewing the manuscript, to all members from different laboratories that help us during field activities and crustacean collections organization and Lourdes M. A. Elmoor-Loureiro and the anonymous reviewers for their important contributions.

\section{Literature Cited}

Almeida, A.O. de and P.A. Coelho. 2008. Estuarine and marine brachyuran crabs (Crustacea: Decapoda) from Bahia, Brazil: checklist and zoogeographical considerations. Latin American Journal of Aquatic Research 36(2): 183-222.

Alves, E. dos S. and P.R. Pezzuto. 2009. Effect of cold fronts on the benthic macrofauna of exposed sandy beaches with contrasting morphodynamics. Brazilian Journal of Oceanography 57(2): 73-96.

Amaral, A. C. Z. and Jablonski, S. 2005. Conservation of marine and coastal biodiversity in Brazil. Conservation Biology 19(3): 625-631.

Ammar, D., Y.M.R. Müller and E.M. Nazari. 2002. Biologia reprodutiva de Macrobrachium potiuna (Müller, 1880) (Crustacea, Decapoda, Palaemonidae) coletados na Ilha de Santa Catarina, Brasil.. Revista Brasileira de Zoologia 18(2): 529-537.

Andersson, Å. 1960. South American terrestrial isopods in the collection of the Swedish State Museum of Natural History. Arkiv för Zoologi 12: $537-570$.

Anker, A. 2012. Revision of the western Atlantic members ofthe Alpheus armillatus H. Milne Edwards, 1837 species complex (Decapoda, Alpheidae), with description of seven new species. Zootaxa 3386: 1-109.

Appeltans W., P. Bouchet, G.A. Boxshall, K. Fauchald, D.P. Gordon, B.W. Hoeksema, G.C.B. Poore, R.W.M. van Soest, S. Stöhr, T.C. Walter and M.J. Costello (ed). 2011. World Register of Marine Species. Eletronic Database accessible at http://www.marinespecies.org. Captured on 7 january 2011.
Athiê, A.A. and C.L. Del B. Rossi-Wongtschowski. 2004. Os caranguejos-deprofundidade na Zona Econômica Exclusiva da Região Sudeste-Sul do Brasil: Análise das operações de pesca e das capturas do N/P "Kinpo Maru ${ }^{\circ}$ 58". São Paulo: Instituto Oceanográfico/USP. 64 p.

Araujo, P.B. de. 1999. Subordem Onscidea (isópodos terrestres, "tatuzinhos"); p. 237-256 In L. Buckup and G. Bond-Buckup (org.). Os crustáceos do Rio Grande do Sul. Porto Alegre: Ed. Universidade/ UFRGS.

Araujo, P.B. and L. Buckup. 1994a. Two new species of terrestrial Isopoda from southern Brasil (Crustacea, Isopoda, Onscidea). Spixiana 17: 269-274.

Araujo, P.B. and L. Buckup. 1994b. Nova espécie de Trichorhina BuddeLund, 1908 (Crustacea, Isopoda, Platyarthridae) do sul do Brasil. Iheringia, Série Zoologia 77: 129-134.

Araujo, P.B. and L. Buckup. 1996a. Novos registros e uma espécie nova de Trichorhina Budde-Lund, (Isopoda, Oniscidea,Platyarthridae) do sul do Brasil. Revista Brasileira de Zoologia 13: 799-810.

Araujo, P.B. and L. Buckup. 1996b. Occorrência de Nagurus Holthuis, 1949 (Isopoda, Trachelipodidae) no sul do Brasil. Nauplius 4: 161-163.

Araujo, P.B. and M. P. Almerão. 2007. Nova espécie de Trichorhina (Isopoda, Oniscidea, Plathyarthridae) do Brasil. Iheringia, Série Zoologia 97(2): 219-222.

Araujo, P.B., L. Buckup and G. Bond-Buckup. 1996. Isópodos terrestres (Crustacea, Oniscidea) de Santa Catarina e Rio Grande do Sul, Brasil. Iheringia, Série Zoologia 81: 111-134.

Baba, K., E. Macpherson, G.C.B. Poore, S.T. Ahyong, A. Bermudez, P. Cabezas, C.W. Lin, M. Nizinski, C. Rodrigues and K.E. Schnabel. 2008. Catalogue of squat lobsters of the world (Crustacea: Decapoda: Anomura - families Chirostylidae, Galatheidae and Kiwaidae). Zootaxa 1905: 1-220.

Barros, M. P. de, J. Brustulin and R. B. Dala Corte. 2007. Heterias (Fritzianira) exul (Müller, 1892) (Janiridae, Isopoda, Crustacea), novo registro para as águas continentais do Rio Grande do Sul, Brasil. Biociências 15(2): 269-270.

Bernardes, R.A., C.L. Del B. Rossi-Wongtschowski, R. Wahrlich, R.C. Vieira, A.P. dos Santos and A.R. Rodrigues. 2005. Prospecção pesqueira de recursos demersais com armadilhas e pargueiras na Zona Econômica Exclusiva da Região Sudeste-Sul do Brasil. São Paulo: Instituto Oceanográfico/USP. 112 p.

Bochi, E. 2000. Species of Decapod crustaceans and their distribution in the American marine zoogeographic provinces. Revista de Investigación y Desarollo Pesquero 13: 7-136.

Bochi, E. and M.A. Gavio. 2005. On the distribution of decapods crustaceans from the Magellan Biogeographic Province and Antarctic region. Scientia Marina 69(suppl. 2): 195-200.

Bond-Buckup, G. 2003. Família Aeglidae; p. 21-116. In G.A.S. Melo (ed.). Manual de identificação dos Crustacea Decapoda de água doce do Brasil. São Paulo: Editora Loyola.

Bond-Buckup, G. and L. Buckup. 1989. Os Palaemonidae de águas continentais do Brasil meridional (Crustacea, Decapoda). Revista Brasileira de Biologia 49(4): 883-896.

Bond-Buckup, G. and L. Buckup. 1999. Caridea (pitus, camarões de água doce e marinhos). P. 300-318. In Bond-Buckup, G. and L. Buckup (org.). Os Crustáceos do Rio Grande do Sul. Porto Alegre: Ed. Universidade/UFRGS. 503p.

Bond-Buckup, G., C.G. Jara, L. Buckup, M. Pérez-Losada, A.A.P. Bueno, K. Crandall and S. Santos. 2010. New species and new records of endemic freshwater crabs from the Atlantic Forest in Southern Brazil (Anomura: Aeglidae). Journal of Crustacean Biology 30(3): 495-502.

Bond-Buckup, G., A. Fransozo, A. do V. Barreto, A.R. Senna, C.U. Magalhães, C.R. Maciel, F.A. Abrunhosa, F. D’Incao, F.L. Mantelatto, L.M.A.ElmoorLoreiro, L. Buckup, M. Tavares, M.L. Negreiros-Fransozo, M. RamosPorto, M. de Sá L. C. de Araújo, N.J. Hebling, O. Rocha, P.B. de Araújo, P.A. Coelho, R.M. Shimizu, W.C. Valenti. 2009. Crustacea; p. 101-130. In Rocha, R.M. da and W.A.P. Boeger (org.). Estado da arte e perspctivas para a Zoologia no Brasil. Curitiba: Sociedade Brasileira de Zoologia - Ed. UFPR.

Boos, H. and S.L. Althoff. 2002. Biologia reprodutiva de Macrobrachium potiuna (Müller, 1880) (Crustacea, Decapoda, Palaemonidae) no Parque Natural Municipal São Francisco de Assis, Blumenau, SC. Estudos de Biologia 24(48): 45-50.

Boos, H. and S.L. Althoff. 2003. Crescimento relativo de Macrobrachium potiuna (Müller, 1880) (Crustacea, Decapoda, Palaemonidae). Estudos de Biologia 25(52): 23-27.

Boos, H., D. Silva-Castiglioni, K. Schacht, L. Buckup and G. Bond-Buckup. 2006. Crescimento de Aegla jarai Bond-Buckup and Buckup (Crustacea, Anomura, Aeglidae). Revista Brasileira de Zoologia 23(2): 490-496.

Boos, H., M.M. de Oliveira and R. Delfim. 2010. Novos registros do siri exótico Charybdis hellerii (A. Milne-Edwards, 1867) (Crustacea, Portunidae), no litoral do Estado de Santa Catarina, Brasil. Revista CEPSUL - Biodiversidade e Conservação Marinha 1: 1-7 
Bott, R. 1969. Die Süsswasserkrabben Süd-Amerikas und ihre Stammesgeschichte. Eine Revision der Trichodactylidae und der Pseudothelphusidae östlich der Anden (Crustacea, Decapoda). Abhandlungen der Senckenbergischen Naturforschenden Gesellschaft 518: 1-94.

Bouzon, J.L. and A.S. Freire, 2007. The Brachyura and Anomura fauna (Decapoda; Crustacea) in the Arvoredo Marine Biological Reserve on the Southern Brazilian Coast. Brazilian Journal of Biology 67(2): 321-325.

Bowman, T.E., R. Prins and J. Arenas. 1987. The occurrence of the freshwater isopod Heterias (Fritzianira) exul in the Lakes Region of Chile, with notes on the genus Heterias (Asellota: Janiridae). Hydrobiologia 146: 275-281.

Boyko, C.B. and P.A. McLaughlin. 2010. Annotated checklist of anomuran decapod crustaceans of the world (exclusive of the Kiwaoidea and families Chirostylidae and Galatheidae of the Galatheoidea) Part IV Hippoidea. The Raffles Bulletin of Zoology 23: 139-151.

Branco, J.0. 1991. Aspectos ecológicos dos Brachyura (Crustacea: Decapoda) no manguezal do Itacorubi, SC - Brasil. Revista Brasileira de Zoologia 7(1-2): 165-179.

Branco, J.O. 2005. Biologia e pesca do camarão sete-barbas Xiphopenaeus kroyeri (Heller) (Crustacea, Penaeidae), na Armação do Itapocoroy, Penha, SC. Revista Brasileira de Zoologia 22(4): 1050-1062.

Branco, J.O. and S. Masunari. 1992. Crescimento de Callinectes danae Smith (Decapoda, Portunidae) da Lagoa da Conceição, Florianópolis, SC, Brazil. Revista Brasileira de Zoologia 9(1/2): 53-66.

Branco, J.O. and H.A.A. Fracasso. 2004. Ocorrência e abundância da carcinofauna acompanhante na pesca do camarão-sete-barbas, Xiphopenaeus kroyeri Heller (Crustacea, Decapoda), na Armação do Itapocoroy, Penha, Santa Catarina, Brasil. Revista Brasileira de Zoologia 21(2): 295-301.

Branco, J.O., M.J. Lunardon-Branco, F.X. Souto and C.R. Guerra. 1999. Population Structure of Sea-Bob-Shrimp Xiphopenaeus kroyeri (Heller, 1862) in Itajaí-Açú Outfall, SC, Brazil. Brazilian Archives of Biology and Technology 42(1): 115-126.

Branco, J.O., H.A.A. Fracasso, F.Freitas Júnior and E. Barbieri. 2011. Biodiversidade no estuário do Saco da Fazenda, Itajaí-SC. O Mundo da Saúde 35(1): 12-22.

Brandão, M., M. Tavares and P.A. Coelho Filho. 2010. A new species of Speocarcinus Stimpson, 1859 from the southwestern Atlantic (Decapoda: Brachyura: Xanthidae). Nauplius 18(2): 137-142.

Brasil-Lima, I.M. and C.M.L. Barros. 1998. Malacostraca - Peracarida. Freshwater Isopoda. Flabellifera and Asellota; p. 645-651 In P.S. Young (ed.). Catalogue of Crustacea of Brazil. Rio de Janeiro: Museu Nacional.

Brasil-Lima, I.M. 1998. Malacostraca - Peracarida. Isopoda. Epicaridea p. 635-644 In P.S. Young (ed.). Catalogue of Crustacea of Brazil. Rio de Janeiro: Museu Nacional.

Buckup, L. 1998. Malacostraca - Eucarida. Astacidea p. 373-375 In P.S. Young (ed.). Catalogue of Crustacea of Brazil. Rio de Janeiro: Museu Nacional.

Buckup, L. 2003. Família Parastacidae; 117-141 In Melo, G.A.S. Manual de identificação dos Crustacea Decapoda de água doce do Brasil. São Paulo: Ed. Loyola.

Buckup, L. and A. Rossi. 1980. 0 gênero Parastacus no Brasil (Crustacea, Decapoda, Parastacidae). Revista Brasileira de Biologia, 40: 663-681.

Calado, T.C.S. 1999. Superfamília Hippoidea (tatuíras, tatuís); p. 406-414 In L. Buckup and G. Bond-Buckup. Os crustáceos do Rio Grande do Sul. Porto Alegre: Ed. Universidade/UFRGS.

Campaner, A.F. 1985. Occurrence and distribution of copepods (Crustacea) in the epipelagial off Southern Brazil. Boletim do Instituto Oceanográfico 33(1): 5-27.

Carvalho, J.L.B., C.A.F. Schettini and T.M. Ribas. 1998. Estrutura termohalina do litoral Centro-Norte catarinense. Notas Técnicas da Faculdade de Ciências do Mar - Univali 2:181-197.

Chan, C.Y. 2010. Annotated checklist of the world's marine lobsters (Crustacea: Decapoda: Astacidea, Glypheidea, Achelata, Polychelida). The Raffles Bulletin of Zoology 23: 153-181.

Christoffersen, M.L. 1998. Malacostraca. Eucarida. Caridea. Crangonoidea and Alpheoidea (Escept Glyphocrangonidae and Crangonidae); p. 351-372. In P.S. Young (ed.). Catalogue of Crustacea of Brazil. Rio de Janeiro: Museu Nacional.

Coelho, P.A. 2006. Revisão de Podochela Stimpson e gêneros afins nas costas caribenha e atlântica da América do Sul (Crustacea, Decapoda, Inachidae). Revista Brasileira de Zoologia 23(3): 678-691.

Coelho, P.A. and M. Ramos-Porto. 1987. Sinopse dos crustáceos decápodos brasileiros (família Callianassidae, Callianeidae, Upogebiidae, Parapaguridae, Paguridae, Diogenidae). Trabalhos Oceanográficos da Universidade Federal de Pernambuco 19: 27-53.

Dall'Occo, P.L., R.T. Bento and G.A.S. de Melo. 2007. Range extensions for lobsters of the brazilian coast (Crustacea, Decapoda, Palinura, Astacidea). Biociências 15(1): 47-52.
De Grave, S., N.D. Pentcheff, S.T. Ahyong, T.Y. Chan, K.A. Crandall, P.C. Dworschak, D.L. Felder, R.M. Feldmann, C.H.J.M. Fransen, L.Y.D. Goulding, R. Lemaitre, M.E.Y. Low, J.W. Martin, P.K.L. Ng, C.E. Schweitzer, S.H. Tan, D. Tshudy and R. Wetzer. 2009. A classification of living and fossil genera of decapod crustaceans. The Raffles Bulletin of Zoology 21: 1-109.

De Grave, S. and C.H.J.M. Fransen. 2011. Carideorum Catalogus: The Recent Species of the Dendrobranchiate, Stenopodidean, Procarididean and Caridean Shrimps (Crustacea: Decapoda). Zoologische Mededelingen 85: $195-588$

D'Incao, F. 1998. Malacostraca. Eucarida. Dendrobranchiata; p. 311-321 In P.S. Young (ed.). Catalogue of Crustacea of Brazil. Rio de Janeiro: Museu Nacional.

D’Incao, F., H. Valentini and L.F. Rodrigues. 2002. Avaliação da pesca de camarões nas regiões Sudeste e Sul do Brasil. 1965-1999. Atlântica 24(2): 49-62.

Elmoor-Loureiro, L.M.A. 2000. Brazilian cladoceran studies: where do we stand? Nauplius 8(1): 117-131.

Emilson, I. 1961. The shelf and coastal Waters off southern Brazil. Boletim do Instituto Oceanográfico de São Paulo 11(2):101-112.

Ferreira, N.C. and A.S. Freire. 2009. Spatio-temporal variation of the pink shrimp Farfantepenaeus paulensis (Crustacea, Decapoda, Penaeidae) associated to the seasonal overtune of the sandbar in a subtropical lagoon. Iheringia, Série Zoologia 99(4): 390-396.

Freitas Jr. et al., F.; M.L. Christoffersen and J.O. Branco. 2010. Monitoring of carcinofauna abundance and diversity during eight years of expressway construction in Santa Catarina, Brazil. Latin american journal of aquatic research 38(3): 461-473.

Gomes-Corrêa, M.M. 1998. Malacostraca. Hoplocarida; p. 289-298 In P.S. Young (ed.). Catalogue of Crustacea of Brazil. Rio de Janeiro: Museu Nacional.

Gomes-Corrêa, M.M. 1999. Ordem Stomatopoda (tamburutacas); p. 144164 In L. Buckup and G. Bond-Buckup (org.). Os crustáceos do Rio Grande do Sul. Porto Alegre: Ed. Universidade/UFRGS.

Guinot, D. and M. Tavares. 2003. A new subfamilial arrangement for the Dromiidae de Haan, 1833, with diagnoses and descriptions of new genera and species (Crustacea, Decapoda, Brachyura. Zoosystema 25(1): 43-129.

Ho, J.S. 1998. Maxillopoda - Copepoda. Siphonostomatoida; p. 251-260 In Young, P.S. (ed.). Catalogue of Crustacea of Brazil. Rio de Janeiro: Museu Nacional.

Integrated Taxonomic Information System. 2010. Eletronic Database accessible at http://www.itis.gov. Captured on 30 may 2010.

Karasawa, H. and C.E. Schweitzer. 2006. A new classification of the Xanthoidea sensu lato (Crustacea: Decapoda: Brachyura) based on phylogenetic analysis and traditional systematics and evaluation of all fossil Xanthoidea sensu lato. Contributions to Zoology $75(1 / 2) 23-$ 73.

Leistikow, A. and J. W. Wägele. 1999. Checklist of the terrestrial isopods of the new world (Crustacea, Isopoda, Oniscidea). Revista Brasileira de Zoologia 16(1): 1-72.

Lemos de Castro, A. and I.M. Brasil-Lima. 1977. Fritzianira, a new genus for Janira exul Müller (Isopoda Asellota). Crustaceana 32(1): 1-6.

Loyola e Silva, J. 1999. Ordem Isopoda (espécies aquáticas); p. 195-236 In L. Buckup and G. Bond-Buckup. Os crustáceos do Rio Grande do Sul. Porto Alegre: Ed. Universidade/UFRGS.

Loyola e Silva, J., S.G. Melo and R. Metri. 2003. Lanocira kroyeri Hansen (Isopoda: Corallidae) redescrição e nova ocorrência para a Ilha Arvoredo, Santa Catarina, Brasil. Acta Biológica Paranaense 32(1, 2, 3, 4): 83-94.

Luque J.L. and L.E.R. Tavares. 2007. Checklist of Copepoda associated with fishes from Brazil. Zootaxa 1579: 1-39.

Machado, I.F., R.R.R. Vieira and F. D’Incao. 2009. Primeiros registros de Brachyura para o litoral de Santa Catarina, Brasil: (Decapoda: Dromiidae, Xanthidae e Inachoididae). Atlântica 31(2): 217-218.

Machado, I.F., R.S. Ferreira and F. D'Incao. 2010. Leander paulensis Ortmann, 1897 e Periclimenes longicauda (Stimpson, 1860) primeiro registro de Caridea (Crustacea, Decapoda, Palaemonidae) para o litoral de Santa Catarina, Brasil. Atlântica 32(1): 119-120.

Magalhães, C. 2003. Famílias Pseudothelphusidae e Trichodactylidae; p. 143-287 In G. A. S. Melo (ed.). Manual de identificação dos Crustacea Decapoda de água doce do Brasil. São Paulo: Editora Loyola.

Magris, R.A., G. Bond-Buckup, C. Magalhães, F.L. Mantelatto, J.W. Reid, L.M.A. El-moor Loureiro, P.A. Coelho, W. Santana, L. Buckup, S.S. da Rocha, S.L.S. Bueno, M.A.A. Pinheiro, F. D’Incao, C.T.C. Ivo, J. Dias Neto, E.S. Rodrigues, P.B. Araujo, H. Boos and L.F.A. Duarte. 2010. Quantification of extinction risk for crustacean species: an overview of the National Red Listing process in Brazil. Nauplius 18(2): 129135.

Malta, J.C., 1998. Maxillopoda. Branchyura; p. 67-74 In P.S. Young (ed.). Catalogue of Crustacea of Brazil. Rio de Janeiro: Museu Nacional.

Mantelatto F.L. and L.L. Dias. 1999. Extension of the known distribution of 
Charybdis hellerii (A. Milne-Edwards, 1867) (Decapoda, Portunidae) along the western tropical South Atlantic. Crustaceana, 72(6): 617620.

Mantelatto F.L.; Pileggi, L.G.; Miranda, I. and I.S. Wehrtmann. 2011. Does Petrolisthes armatus (Anomura, Porcellanidae) form a species complex or are we dealing with just one widely distributed species? Zoological Studies 50(3): 372-384.

Markham, J.C. 1988. Descriptions and revisions of some species of Isopoda Bopyridae of the north western Atlantic Ocean. Zoologische Verhandelingen 246: 1-63.

Martin, J.W. and G.E. Davis. 2001. An updated classification of the recent Crustacea. Los Angeles: Natural History Museum of Los Angeles County. $124 \mathrm{p}$.

Martins, S.T.S. and F. D'Incao. 1996. Os Pinnotheridae de Santa Catarina e Rio Grande do Sul, Brasil (Decapoda, Brachyura). Revista Brasileira de Zoologia 13(1): 1-26.

Martens, K., N.L. Würdig and F. Behen. 1998. Maxillopoda, non-marine Ostracoda; p. 45-65. In P. S. Young (ed.). Catalogue of Crustacea of Brazil. Rio de Janeiro: Museu Nacional.

McLaughlin, P.A., T. Komai, R. Lemaitre and D.L. Rahayu. 2010a. Annotated checklist of anomuran decapod crustaceans of the world (exclusive of the Kiwaoidea and families Chirostylidae and Galatheidae of the Galatheoidea) Part I - Lithodoidea, Lomisoidea and Paguroidea. The Raffles Bulletin of Zoology 23: 5-107.

McLaughlin, P.A., R. Lemaitre and K.A. Crandall. 2010b. Annotated checklist of anomuran decapod crustaceans of the world (exclusive of the Kiwaoidea and families Chirostylidae and Galatheidae of the Galatheoidea) Part III - Aegloidea. The Raffles Bulletin of Zoology 23: 131-137.

Melo, G.A.S. 1967. Diferenciação geográfica e dimorfismo sexual de Trichodactylus (Trichodactylus) fluviatilis Latreille, 1825 (Crustacea, Brachyura). Papéis Avulsos de Zoologia 20 (3): 13-44

Melo, G.A.S. 1990. A presença, no litoral Sudeste brasileiro, de espécies de Brachyura (Crustacea: Decapoda) originárias das regiões biogeográficas Magelânica e Argentina do Atlântico Sul. Atlântica 12(2): 71-83.

Melo, G.A.S. 1996. Manual de identificação dos Brachyura (caranguejos e siris) do Litoral brasileiro. São Paulo: Plêiade/FAPESP.

Melo, G.A.S. 1999. Infraordem Brachyura (siris e caranguejos: espécies marinhas e estuarinas); p. 415-485 In L. Buckup and G. BondBuckup (org.). Os crustáceos do Rio Grande do Sul. Porto Alegre: Ed. Universidade/UFRGS.

Melo, G.A.S. 2003. Famílias Atyidae, Palaemonidae e Sergestidae; p. 289-415 In G.A.S. Melo (ed.). Manual de identificação dos Crustacea Decapoda de água doce do Brasil. São Paulo: Editora Loyola.

Melo, G.A.S. 2010. The brachyura (Cruastacea: Decapoda) collected by the GEDIP Project between Torres, Rio Grande do Sul (Brazil) and Maldonado (Uruguay). Atlântica 32(1): 39-57.

Melo, G.A.S. and O. Campos Jr. 1999. A família Dromiidae De Haan no litoral brasileiro, com descrição de uma nova espécie (Crustacea: Decapoda: Brachyura). Revista Brasileira de Zoologia 16(2): 273-291.

Melo, G.A.S. and Boehs, G. 2004. Rediscovery of Holothuriophilus tomentosus (Ortmann) comb. nov. (Crustacea, Brachyura, Pinnotheridae) in the Brazilian coast. Revista Brasileira de Zoologia 21(2): 229-232.

Melo-Filho, G.A.S. 1999. Famílias Chirostylida e Galatheidae; p.383-397 In L. Buckup and G. Bond-Buckup (org.). Os crustáceos do Rio Grande do Sul. Porto Alegre: Ed. Universidade/UFRGS

Melo-Filho, G.A.S. 2006. Reports on the results of the N.Oc. "Prof. W. Besnard" expeditions to the southern coast of Brazil under the Revizee Program: Chirostylidae and Galatheidae (Crustacea: Decapoda: Anomura). Zootaxa 1238: 1-22.

Melo-Filho, G.A.S. 2008. Specimens of Galatheidae (Crustacea, Decapoda) collected by NPq. "Soloncy Moura" off the Southern Coast of Brazil under the Revizee Program. Zootaxa 1841: 31-42.

Melo-Filho, G.A.S. and G.A.S. Melo. 2001. Espécies do gênero Munida Leach (Crustacea, Decapoda, Galatheidae), distribuídas na costa do Brasil. Revista Brasileira de Zoologia 18 (4): 1135 -1176.

Montú, M. and I.M. Gloeden. 1998. Branchiopoda. Marine "Cladocera"; p. 11-14 In P. S. Young (ed.). Catalogue of Crustacea of Brazil. Rio de Janeiro: Museu Nacional.

Moreira, C. 1903. Crustáceos da Ponta do Pharol em São Francisco do Sul, no Estado de Santa Catarina. Archivos do Museu Nacional 12: 119123.

Müller, F. 1862. Entoniscus porcellanae, eine neue schmarotzerassel. Archiv für Naturgeschicte 1: 10-18

Muller, F. 1876. Aegla odebrechtii n. sp. Jen. Zeitsch. Naturwis., N.S., 10(3): 13-24.

Müller, F. 1880a. Palaemon potiuna. Ein Beispiel abgekurster Verwandbung. Zool. Anz., 3: 152-157.

Müller, F. 1880b. Wasserthiere in Baumwipfeln: Elpidium bromeliarum. Kosmos 4: 386-388.
Müller, F. 1881. Atyoida potimirim, eine schlammfressende Susswassergarneele. Kosmos 9: 117-124.

Müller, F. 1892. Descrição de Janira exul, crustáceo isópode do Estado de Santa Catarina. Archivos do Museu Nacional 8: 207-220.

Müller, Y.M.R. and J. Araujo. 1994. Dados biologicos de Palaemonetes argentinus (Decapoda, Palaemonidae) coletados no canal do Rio Ratones - Florianopolis. Revista Brasileira de Biologia 54(3): 55-64.

Müller, Y.M.R. and J. Mittmann. 1998. Contribution to the knowledge of Caprella penantis (Leach) (Crustacea, Amphipoda) in Anhatomirim Island, Santa Catarina. Revista Brasileira de Zoologia 15(1): 95-100.

Müller, Y.M.R., C.M. Bressan and E.M. Nazari. 1993. Dados de fecundidade de Macrobrachium acanthurus (Decapoda, Palemonidae) do Rio Capivari, Praia dos Ingleses, Florianopolis - SC. Biotemas 5(1): 123 132.

Müller, Y.M.R., E.M. Nazari, C.M. Bressan and D. Ammar. 1996. Aspectos da reprodução de Palaemon pandaliformis (Decapoda, Palaemondae) no manguezal de Ratones - Florianopolis SC. Revista Brasileira de Zoologia 13(3): 633-642.

Nazari, E.M., M.S.S. Costa, Y.M.R. Müller, D. Ammar and M. Dias. 2003. Comparisons of fecundity, egg size and egg mass volume of the freshwater prawns Macrobrachium potiuna and Macrobrachium olfersi (Decapoda, Palaemonidae). Journal of Crustacean Biology 23(4): 862-868.

Ng P.K.L, D. Guinot and P.J.F. Davie. 2008. Systema Brachyurorum: Part I. an annotated checklist of extant brachyuran crabs of the world. The Raffles Bulletin of Zoology 17:1-286.

Nucci, P.R., G.A.S. de Melo, G.A.S. de Melo-Filho and O. Campos Jr. 2004. Classe Malacostraca Ordens Decapoda and Stomatopoda; p. 136140. In A. C. Z. Amaral and C. L. Del B. Rossi-Wongtschowski (ed.) Biodiversidade Bentônica da Região Sudeste-Sul do Brasil - Plataforma Externa e Talude Superior. São Paulo: Instituto Oceanográfico/USP.

Oliveira, G., A.S. Freire and P.R.K. Bertuol. 2008. Reproductive biology of the slipper lobster Scyllarides deceptor (Decapoda: Scyllaridae) along the Southern Brazilian Coast. Journal of the Marine Biological Association of the UK 88(7): 1433-1440.

Quadros, A.F. and P.B. Araujo. 2008. An assemblage of terrestrial isopods (Crustacea) in southern Brazil and its contribution to leaf litter processing. Revista Brasileira de Zoologia 25 (1): 58-66.

Ramos-Porto, M. and Coelho, P.A. 1998. Malacostraca. Eucarida. Caridea (Alpheoidea excluded); p. 325-350 In P.S. Young (ed.). Catalogue of Crustacea of Brazil. Rio de Janeiro: Museu Nacional.

Reid, J.W, 1998. Maxillopoda - Copepoda. Harpacticoida; p. 75 - 127 In P.S Young (ed.). Catalogue of Crustacea of Brazil. Rio de Janeiro: Museu Nacional.

Resgala Jr, C. 2001. Estudos de impacto ambiental na comunidade do zooplâncton na Enseada do Saco dos Limões, Baía Sul da Ilha de Santa Catarina, Brasil. Atlântica 23: 5-16.

Resgalla Jr, C., V.G.C. de Souza, L.R. Rörig, C.A.F. Schettini. 2008. Spatial and temporal variation of the zooplankton community in the area of influence of the Itajaí-açú Rio, SC (Brazil). Revista Brasileira de Oceanografia 56(3): 211-224

Rieger, P.J. 1997. Os "ermitões" (Crustacea, Decapoda, Parapaguridae, Diogenidae e Paguridae) do Litoral do Brasil. Nauplius 5(2): 99-124.

Rieger, P.J. and J.B. Giraldi. 1996. Mithraculus forceps (Edwards, 1875) novo registro de Brachyura (Decapoda, Majidae) para o litoral do Estado de Santa Catarina, Brasil. Trabalhos Oceanográficos da Universidade Federal de Pernambuco 24: 237-240.

Rieger, P.J. and J.B. Giraldi. 1997a. Calcinus tibicen (Herbst, 1791) e Paguristes tortugae Schmitt, 1933, novo registro de Diogenidae (Crustacea, Anomura) para o litoral de Santa Catarina, Brasil. Nauplius 5(2): 157-159.

Rieger, P.J. and J.B. Giraldi. 1997b. Nota sobre nova ocorrência de Pagurus brevidactylus Stimpson, 1859 (Crustacea, Anomura, Paguridae) no litoral brasileiro. Revista Brasileira de Zoologia 14(2): 511-512.

Rieger, P.J. and J.B. Giraldi. 2001. Mithrax hispidus (Herbst) e Mithrax tortugae Rathbun novos registros de Brachyura (Decapoda, Majidae) para o litoral de Santa Catarina, Brasil. Revista Brasileira de Zoologia 18(2): 653-654.

Rocha, C.E.F. and M.J.C. Botelho. 1998. Maxilopoda - Copepoda. Cyclopoida; p. 129-166 In P.S. Young (ed.). Catalogue of Crustacea of Brazil. Rio de Janeiro: Museu Nacional.

Rocha, R.M. da and W.A.P. Boeger (org.). 2009. Estado da arte e perspctivas para a Zoologia no Brasil. Curitiba: Sociedade Brasileira de Zoologia - Ed. UFPR. 296 p.

Rodrigues, C. and P.S. Young. 2005. Stomtopoda (Crustacea, Hoplocarida) coletados pelo Programa Revizee com duas novas ocorrências para a costa do Brasil. Arquivos do Museu Nacional 63(2): 233-245.

Santana, W., A.P. Pinheiro and J.E.L. Oliveira. 2007. Additional records of three Scyllarides species (Palinura: Scyllaridae) from Brazil, with the description of the fourth larval stage of Scyllarides aequinoctialis. Nauplius 15(1): 1-6.

Santana, W. and M. Tavares. 2009. Podochela meloi Sankarankutty, 
Ferreira \& Cunha, 2001, a junior synonym of the spider crab Inachoides forceps A. Milne-Edwards, 1879 (Crustacea: Brachyura: Inachoididae). Zootaxa 2294: 62-68.

Santos, S., G. Bond-Buckup, L. Buckup, M. Pérez-Losada, M. Finley and K.A.Crandall. 2012. Three new species of Aegla (Anomura) freshwater crabs from the upper Uruguay river hydrographic basin in brazil. Journal of Crustacean Biology 32(4): 529-540.

Santos-Silva, E.N. 1998. Maxillopoda - Copepoda: Freshwater Calanoida; p. 201-220 In P.S. Young (ed.). Catalogue of Crustacea of Brazil. Rio de Janeiro: Museu Nacional.

Schaffer-Novelli, Y., G. Cintrón-Molero, R.R. Adaime and T.M. de Camargo. 1990. Variability of mangrove ecossystems along the brazilian coast. Estuaries 13(2): 204-218.

Serafim-Júnior, M., G.P. Neves, L. de Brito and A.R. Ghidini. 2006. Zooplâncton do Rio Itajaí-Acú a jusante da cidade de Blumenau, Santa Catarina, Brasil. Estudos de Biologia 28(65): 41-50.

Severino, A. and C. Resgalla Jr. 2005. Descrição dos estágios larvais de Megabalanus coccopoma (Drawin, 1854) e sua variação temporal na enseada de Itapocoroy (Santa Catarina, Brasil). Atlântica 27(1): 5-16.

Soto, J.M.R. and M.M. Mincarone. 2001. Distribution and morphology of the giant isopods Bathynomus giganteus and Bathynomus miyarei (Flabellifera, Cirolanidae) of Southern Brazil. Mare Magna 1(2): 141145.

Souza-Kury, L.A. 1998. Malacostraca - Peracarida. Isopoda. Oniscidea; p. 653-674 In P.S. Young (ed.). Catalogue of Crustacea of Brazil. Rio de Janeiro: Museu Nacional.

Tan, S.H. and P.K.L. Ng. 2007. Descriptions of new genera from the subfamily Parthenopinae (Crustacea: Decapoda: Brachyura: Parthenopidae). The Raffles Bulletin of Zoology 16: 95-119.

Tavares, M. 1996. Révision systématique des Cyclodorippidae américains (Crustacea, Decapoda, Brachyura). Bulletin du Muséum National d'Histoire Naturelle 18(1-2): 233-295.

Tavares, M. 2002a. Stomatopods; p. 245-250 In K.E. Carpenter (ed.). The Living Marine Resources of the Western Central Atlantic. Volume I. Rome: FAO.

Tavares, M. 2002b. Shrimps; p. 251-291 In K.E. Carpenter (ed.). The Living Marine Resources of the Western Central Atlantic. Volume I. Rome: FAO.

Tavares, M. 2002c. True Crabs; p. 327-352 In K.E. Carpenter (ed.). The Living Marine Resources of the Western Central Atlantic. Volume I. Rome: FAO.

Tavares, M. and Cleva, R. 2010. Trichopeltariidae (Crustacea, Decapoda, Brachyura), a new family and superfamily of eubrachyuran crabs with description of one new genus and five new species. Papéis Avulsos de Zoologia 50(9): 97-157.

Tavares, M. and Melo, G.A.S. 2005. A new species of Trichopeltarion A. Milne-Edwards, 1880, from the Southwestern Atlantic (Crustacea: Brachyura: Atelecyclidae). Papéis Avulsos de Zoologia 45(18): 235242 .
Tavares, M. and Pinheiro, A.P. 2011. A new species of Chaceon Manning \& Holthuis, 1989, from the southwestern Atlantic, with a key to the western Atlantic species (Crustacea, Decapoda, Geryonidae). Zootaxa 3086: 57-68.

Tavares, M., W. Santana and A. Pinheiro. 2009. On the type of Scyllarides deceptor Holthuis, 1963 (Crustacea: Decapoda: Scyllaridae). Papéis Avulsos de Zoologia 49(40): 539-545.

Terossi, M. and F.L. Mantelatto. 2012 Morphological and genetic variability in Hippolyte obliquimanus Dana, 1852 (Decapoda: Caridea: Hippolytidae) from Brazil and the Caribbean sea. Crustaceana 85(6): 685-712.

Thatcher V.E. and G.M. Huergo. 2005. Minilernaea floricapitella gen. nov., sp. nov. (Copepoda, Lernaeidae) from freshwater fishes of Southern Brazil. Revista Brasileira de Zoologia 22(4): 824-824.

Torati, L.S. and F.L. Mantelatto. 2012. Ontogenetic and evolutionary change of external morphology of the Neotropical shrimp Potimirim (Holthuis, 1954) explained by a molecular phylogeny of the genus. Journal of Crustacean Biology 32(4): 625-640.

Valério-Berardo M.T. and V.K. Miyagi. 2000. Corophiidae (Crustacea, Amphipoda) da costa brasileira. Revista Brasileira de Zoologia 17 (2): 481 - 504.

Veado, L.D. and C. Resgalla Jr. 2005. Alteração da comunidade zooplanctônica do saco dos Limões após impacto das obras da via expressa sul - Baía Sul da Ilha de Santa Catarina. Brazilian Journal of Aquatic Science and Technology 9(2): 65-73.

Veado, L. D., B.S. Sant'ana and C. Resgalla Jr. 2010. Atlas do zooplâncton dominante no baixo estuário do Rio Itajaí-Açu, Santa Catarina, Brasil: Copepoda e Cladocera. Brazilian Journal of Aquatic Sciences and Technology 10(2): 79-93.

Veloso, V.G. 1999. Família Porcellanidae (caranguejos anomuros marinhos); p. 398-405 In L. Buckup and G. Bond-Buckup. Os crustáceos do Rio Grande do Sul. Porto Alegre: Ed. Universidade/ UFRGS.

Veloso, V.G. and G.A.S. de Melo. 1993. Taxonomia e distribuição da família Porcellanidae (Crustacea, Decapoda, Anomura) no litoral brasileiro. Iheringia, Série Zoologia 75: 171-186.

Young, P.S. 1998. Maxillopoda. Thecostraca; p. 263-285 In P.S. Young (ed.). Catalogue of Crustacea of Brazil. Rio de Janeiro: Museu Nacional.

Young, P.S. 1999. Subclasse Cirripedia (cracas); p. 24-53 In L. Buckup and G. Bond-Buckup. Os crustáceos do Rio Grande do Sul. Porto Alegre: Ed. Universidade/UFRGS.

Wakabara, Y. and C.S. Serejo, 1998. Malacostraca - Peracarida. Amphipoda Gammaridea and Caprellidea; p. 561-594 In P.S. Young (ed.) Catalogue of Crustacea of Brazil. Rio de Janeiro: Museu Nacional.

RECEIVED: April 2012

ACCEPTED: August 2012

Published ONLINE: November 2012

EDITORIAL RESPONSIBILITY: Luis Ernesto Arruda Bezerra 